\title{
Palladium-Catalyzed Heteroannulation of Indole-1- carboxamides with [60]Fullerene and Subsequent Electrochemical Transformations
}

\author{
Majid Hussain, ${ }^{\dagger}$ Muqing Chen, ${ }^{*},+$ Shangfeng Yang, ${ }^{\ddagger}$ and Guan-Wu Wang ${ }^{*}, \dagger$,
}

${ }^{\dagger}$ CAS Key Laboratory of Soft Matter Chemistry, Hefei National Laboratory for Physical Sciences at Microscale, and Department of Chemistry, University of Science and Technology of

China, Hefei, Anhui 230026, P. R. China

†Hefei National Laboratory for Physical Sciences at Microscale, CAS Key Laboratory of Materials for Energy Conversion, and Department of Materials Science and Engineering, University of Science and Technology of China, Hefei, Anhui 230026, P. R. China

$₫$ State Key Laboratory of Applied Organic Chemistry, Lanzhou University, Lanzhou, Gansu 730000, P. R. China

\section{Table of Contents}

1. General procedure for the synthesis of $2 \mathbf{a}-\mathbf{n} \quad$ S2

2. Synthesis and spectral data of compounds $2 a-n \quad$ S2

3. Synthesis and spectral data of compounds 3a-d $\quad$ S11

$\begin{array}{ll}\text { 4. Scaled-up synthesis of } 2 \mathrm{a} & \text { S15 }\end{array}$

$\begin{array}{ll}\text { 5. Mechanistic Study } & \text { S15 }\end{array}$

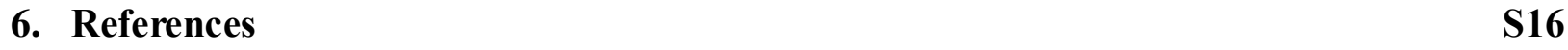

7. NMR spectra of compounds $2 a-n, 3 a-d$, and [D]-1a

8. UV-vis spectra of compounds $2 a-n$ and $3 a-d \quad S 45$

9. CV of compounds $2 a-n$ and 3a-d $\quad$ S50

10. X-Ray data of compound 2e

$\begin{array}{lr}\text { 11. X-Ray data of compound 3d } & \text { S58 }\end{array}$ 


\section{General procedure for the synthesis of $2 \mathbf{a}-\mathbf{n}$}

A mixture of $\mathrm{C}_{60}(0.05 \mathrm{mmol}), 1(0.15 \mathrm{mmol}), \mathrm{Pd}(\mathrm{OAc})_{2}(0.005 \mathrm{mmol}), \mathrm{K}_{2} \mathrm{~S}_{2} \mathrm{O}_{8}(0.10 \mathrm{mmol})$, and trifluoroacetic acid (TFA) (0.2 mL) was dissolved in ortho-dichlorobenzene (ODCB) (5 mL). Then the solution was stirred at the desired temperature and stopped at the designated time. The resulting solution was evaporated in vacuo and then separated on a silica gel column with $\mathrm{CS}_{2} / \mathrm{CH}_{2} \mathrm{Cl}_{2}$ (4:1 $\mathrm{v} / \mathrm{v})$ as the eluent to give recovered $\mathrm{C}_{60}$ and the desired product 2.

\section{Synthesis and spectral data of compounds $2 a-n$} Synthesis and spectral data of compound 2 a

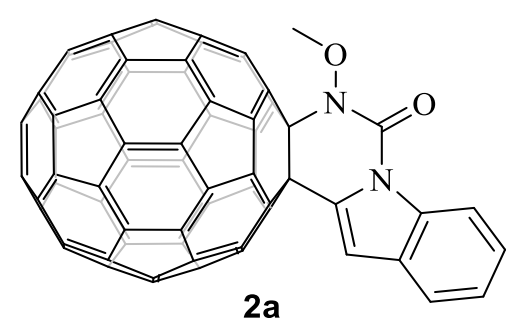

Preparation of 2a: By following the general procedure, the reaction of $\mathrm{C}_{60}(36.0 \mathrm{mg}, 0.05 \mathrm{mmol})$ with $1 \mathrm{a}(28.5 \mathrm{mg}, 0.15 \mathrm{mmol}), \mathrm{Pd}(\mathrm{OAc})_{2}(1.1 \mathrm{mg}, 0.005 \mathrm{mmol}), \mathrm{K}_{2} \mathrm{~S}_{2} \mathrm{O}_{8}(27.2 \mathrm{mg}, 0.10 \mathrm{mmol})$, and TFA $(0.2 \mathrm{~mL})$ in $\mathrm{ODCB}(5 \mathrm{~mL})$ at $100{ }^{\circ} \mathrm{C}$ for $28 \mathrm{~h}$ afforded recovered $\mathrm{C}_{60}(18.8 \mathrm{mg}, 49 \%)$ and 2a (14.8 mg, 33\%): amorphous brown solid; ${ }^{1} \mathrm{H} \mathrm{NMR}\left(400 \mathrm{MHz}, \mathrm{CS}_{2} / \mathrm{CDCl}_{2} \mathrm{CDCl}_{2}, 2: 3\right) \delta 8.57$ $(\mathrm{d}, J=8.3 \mathrm{~Hz}, 1 \mathrm{H}), 7.66(\mathrm{~d}, J=7.7 \mathrm{~Hz}, 1 \mathrm{H}), 7.52$ (t, $J=7.8 \mathrm{~Hz}, 1 \mathrm{H}), 7.39$ (t, $J=7.5 \mathrm{~Hz}, 1 \mathrm{H}), 7.35$ (s, 1H), $4.14(\mathrm{~s}, 3 \mathrm{H}) ;{ }^{13} \mathrm{C}$ NMR $\left(101 \mathrm{MHz}, \mathrm{CS}_{2} / \mathrm{CDCl}_{3}, 2: 3\right) \delta 149.98,148.13,147.77,146.59$, $146.44,146.26,146.20,146.06,145.92,145.50,145.32$, 145.25, 145.15, 144.57, 144.53, 142.90, $142.73,142.67,142.33,142.16,142.13,141.75,141.56,141.22,140.50,138.98,137.17,136.24$, 134.14, 129.96, 125.83, 124.30, 121.07, 116.11, 110.23, 80.57, 65.11, 60.79; FT-IR v/cm ${ }^{-1}$ (KBr) 2923, 2843, 1719, 1510, 1448, 1363, 1221, 1033, 992, 842, 809, 744, 530; UV-vis $\left(\mathrm{CHCl}_{3}\right)$ $\lambda_{\max } / \mathrm{nm}(\log \varepsilon) 254$ (5.00), 316 (4.57), 412 (3.66), 460 (3.41); MALDI-TOF MS m/z calcd for $\mathrm{C}_{70} \mathrm{H}_{8} \mathrm{~N}_{2} \mathrm{O}_{2}[\mathrm{M}]^{+} 908.0580$, found 908.0589 .

\section{Synthesis and spectral data of compound $2 b$}




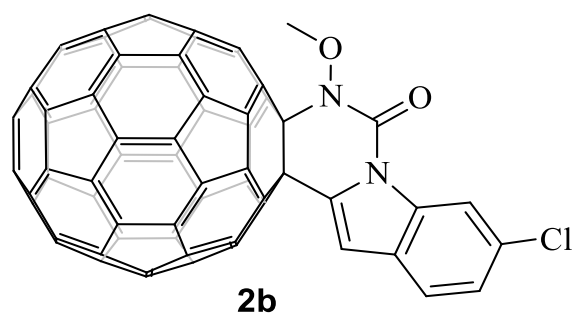

Preparation of $\mathbf{2 b}$ : By following the general procedure, the reaction of $\mathrm{C}_{60}(36.0 \mathrm{mg}, 0.05 \mathrm{mmol})$ with $1 \mathrm{~b}$ (33.4 mg, $0.15 \mathrm{mmol}), \mathrm{Pd}(\mathrm{OAc})_{2}(1.2 \mathrm{mg}, 0.005 \mathrm{mmol}), \mathrm{K}_{2} \mathrm{~S}_{2} \mathrm{O}_{8}(27.1 \mathrm{mg}, 0.10 \mathrm{mmol})$, and TFA $(0.2 \mathrm{~mL})$ in $\mathrm{ODCB}(5 \mathrm{~mL})$ at $100{ }^{\circ} \mathrm{C}$ for $36 \mathrm{~h}$ afforded recovered $\mathrm{C}_{60}(18.5 \mathrm{mg}, 52 \%)$ and 2b (16.5 mg, 35\%): amorphous brown solid; ${ }^{1} \mathrm{H}$ NMR (400 MHz, $\left.\mathrm{CS}_{2} / \mathrm{CDCl}_{2} \mathrm{CDCl}_{2}, 2: 3\right) \delta 8.62$ (d, $J=1.9 \mathrm{~Hz}, 1 \mathrm{H}), 7.58(\mathrm{~d}, J=8.3 \mathrm{~Hz}, 1 \mathrm{H}), 7.37$ (dd, $\left.J_{1}=8.3 \mathrm{~Hz}, J_{2}=1.9 \mathrm{~Hz}, 1 \mathrm{H}\right), 7.33(\mathrm{~s}, 1 \mathrm{H})$, $4.15(\mathrm{~s}, 3 \mathrm{H})$; The ${ }^{13} \mathrm{C}$ NMR spectrum of $\mathbf{2} \mathbf{b}$ with good $\mathrm{S} / \mathrm{N}$ ratio could not be obtained due to its low solubility. FT-IR $v / \mathrm{cm}^{-1}(\mathrm{KBr}) 2920$, 2849, 1742, 1651, 1572, 1524, 1436, 1380, 1260, 1112 , 1030, 880, 818, 527; UV-vis $\left(\mathrm{CHCl}_{3}\right) \lambda_{\max } / \mathrm{nm}(\log \varepsilon) 255$ (5.01), 318 (4.62), 411 (3.43), 457 (3.15); MALDI-TOF MS $m / z$ calcd for $\mathrm{C}_{70} \mathrm{H}_{7}^{35} \mathrm{ClN}_{2} \mathrm{O}_{2}$ [M] 942.0202, found 942.0203.

\section{Synthesis and spectral data of compound 2c}

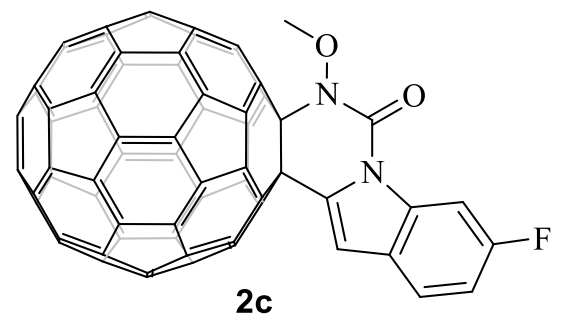

Preparation of 2c: By following the general procedure, the reaction of $\mathrm{C}_{60}(36.0 \mathrm{mg}, 0.05 \mathrm{mmol})$ with 1 c (31.5 mg, $0.15 \mathrm{mmol}), \mathrm{Pd}(\mathrm{OAc})_{2}(1.1 \mathrm{mg}, 0.005 \mathrm{mmol}), \mathrm{K}_{2} \mathrm{~S}_{2} \mathrm{O}_{8}(27.0 \mathrm{mg}, 0.10 \mathrm{mmol})$, and TFA $(0.2 \mathrm{~mL})$ in $\mathrm{ODCB}(5 \mathrm{~mL})$ at $100{ }^{\circ} \mathrm{C}$ for $32 \mathrm{~h}$ afforded recovered $\mathrm{C}_{60}(20.6 \mathrm{mg}, 57 \%)$ and 2 c (11.4 mg, 25\%): amorphous brown solid; ${ }^{1} \mathrm{H} \mathrm{NMR}\left(400 \mathrm{MHz}, \mathrm{CS}_{2} / \mathrm{CDCl}_{2} \mathrm{CDCl}_{2}, 2: 3\right) \delta 8.31$ $\left(\mathrm{dd}, J_{1}=9.6 \mathrm{~Hz}, J_{2}=2.4 \mathrm{~Hz}, 1 \mathrm{H}\right), 7.59\left(\mathrm{dd}, J_{1}=8.6 \mathrm{~Hz}, J_{2}=5.3 \mathrm{~Hz}, 1 \mathrm{H}\right), 7.32(\mathrm{~d}, J=0.6 \mathrm{~Hz}, 1 \mathrm{H})$, $7.13\left(\mathrm{td}, J_{1}=9.2 \mathrm{~Hz}, J_{2}=2.4 \mathrm{~Hz}, 1 \mathrm{H}\right), 4.16(\mathrm{~s}, 3 \mathrm{H}) ;{ }^{13} \mathrm{C} \mathrm{NMR}\left(101 \mathrm{MHz}, \mathrm{CS}_{2} / \mathrm{CDCl}_{2} \mathrm{CDCl}_{2}, 2: 3\right)$ $\delta 160.42(\mathrm{~d}, J=243.5 \mathrm{~Hz}), 149.06,147.23,146.86,145.66,145.51,145.34,145.29,145.14,145.00$, 144.74, 144.53, 144.39, 144.32, 144.22, 143.61, 143.54, 141.95, 141.78, 141.72, 141.36, 141.21, $141.10,140.79,140.61,140.19,139.58,138.07,136.22(\mathrm{~d}, J=12.8 \mathrm{~Hz}), 135.33,133.82(\mathrm{~d}, J=$ $4.6 \mathrm{~Hz}), 125.43$ (d, $J=1.3 \mathrm{~Hz}), 121.09$ (d, $J=9.6 \mathrm{~Hz}), 111.76$ (d, $J=23.5 \mathrm{~Hz}), 108.97,102.58$ (d, $J=28.5 \mathrm{~Hz}), 79.71,64.73,59.71$; FT-IR $v / \mathrm{cm}^{-1}(\mathrm{KBr}) 2916,2855,1718,1656,1483,1438,1374$, 
1256, 1100, 1033, 845, 803, 526; UV-vis $\left(\mathrm{CHCl}_{3}\right) \lambda_{\max } / \mathrm{nm}(\log \varepsilon) 257$ (4.99), 315 (4.54), 408 (3.68), 442 (3.41); MALDI-TOF MS $m / z$ calcd for $\mathrm{C}_{70} \mathrm{H}_{7} \mathrm{FN}_{2} \mathrm{O}_{2}$ [M] 926.0497 , found 926.0495.

\section{Synthesis and spectral data of compound 2d}

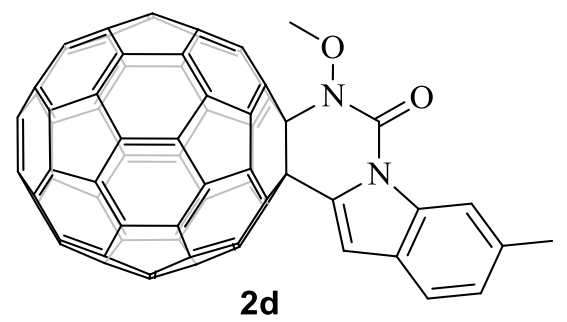

Preparation of $\mathbf{2 d}$ : By following the general procedure, the reaction of $\mathrm{C}_{60}(36.1 \mathrm{mg}, 0.05 \mathrm{mmol})$ with 1 d $(30.8 \mathrm{mg}, 0.15 \mathrm{mmol}), \mathrm{Pd}(\mathrm{OAc})_{2}(1.2 \mathrm{mg}, 0.005 \mathrm{mmol}), \mathrm{K}_{2} \mathrm{~S}_{2} \mathrm{O}_{8}(26.7 \mathrm{mg}, 0.10 \mathrm{mmol})$, and TFA $(0.2 \mathrm{~mL})$ in ODCB $(5 \mathrm{~mL})$ at $100{ }^{\circ} \mathrm{C}$ for $28 \mathrm{~h}$ afforded recovered $\mathrm{C}_{60}(16.8 \mathrm{mg}, 47 \%)$ and 2d (13.9 mg, 30\%): amorphous brown solid; ${ }^{1} \mathrm{H}$ NMR (400 MHz, $\left.\mathrm{CDCl}_{2} \mathrm{CDCl}_{2}\right) \delta 8.41(\mathrm{~s}, 1 \mathrm{H})$, 7.54 (d, $J=7.9 \mathrm{~Hz}, 1 \mathrm{H}), 7.30$ (s, 1H), 7.22 (d, $J=7.9 \mathrm{~Hz}, 1 \mathrm{H}), 4.14$ (s, 3H), $2.58(\mathrm{~s}, 3 \mathrm{H}) ;{ }^{13} \mathrm{C}$ NMR (101 MHz, $\left.\mathrm{CDCl}_{2} \mathrm{CDCl}_{2}\right) \delta 149.55,147.22,146.83,145.63,145.46,145.30,145.24,145.11$, 144.78, 144.54, 144.33, 144.30, 144.19, 143.62, 143.52, 141.90, 141.74, 141.67, 141.33, 141.20, 141.12 , 140.76, 140.58, 140.20, 139.54, 138.01, 136.55, 135.38, 135.36, 132.96, 126.85, 124.97, 119.97, 115.19, 109.39, 79.67, 64.77, 59.85, 21.16; FT-IR $v / \mathrm{cm}^{-1}$ (KBr) 2917, 2852, 1719, 1433, 1366, 1315, 1268, 1109, 1036, 842, 818, 765, 741, 524; UV-vis $\left(\mathrm{CHCl}_{3}\right) \lambda_{\max } / \mathrm{nm}(\log \varepsilon) 254$ (5.00), 314 (4.57), 410 (3.68), 455 (3.43); MALDI-TOF MS $m / z$ calcd for $\mathrm{C}_{71} \mathrm{H}_{10} \mathrm{~N}_{2} \mathrm{O}_{2}[\mathrm{M}]^{-} 922.0748$, found 922.0755 .

\section{Synthesis and spectral data of compound $2 \mathrm{e}$}

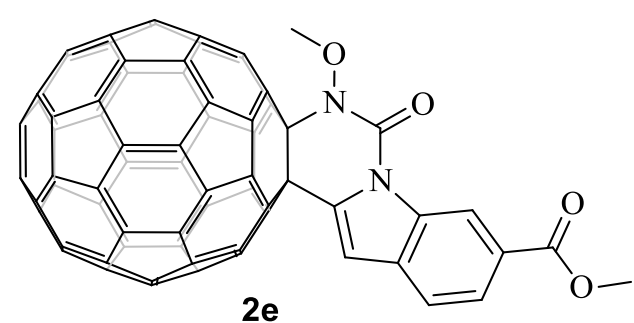

Preparation of 2e: By following the general procedure, the reaction of $\mathrm{C}_{60}(35.9 \mathrm{mg}, 0.05 \mathrm{mmol})$ with $1 \mathrm{e}(37.1 \mathrm{mg}, 0.15 \mathrm{mmol}), \mathrm{Pd}(\mathrm{OAc})_{2}(1.2 \mathrm{mg}, 0.005 \mathrm{mmol}), \mathrm{K}_{2} \mathrm{~S}_{2} \mathrm{O}_{8}(27.4 \mathrm{mg}, 0.10 \mathrm{mmol})$, and TFA $(0.2 \mathrm{~mL})$ in ODCB $(5 \mathrm{~mL})$ at $90{ }^{\circ} \mathrm{C}$ for $32 \mathrm{~h}$ afforded recovered $\mathrm{C}_{60}(15.9 \mathrm{mg}, 44 \%)$ and 
2e (15.2 mg, 31\%): amorphous brown solid; ${ }^{1} \mathrm{H}$ NMR (400 MHz, $\left.\mathrm{CDCl}_{2} \mathrm{CDCl}_{2}\right) \delta 9.22(\mathrm{~s}, 1 \mathrm{H})$, $8.06(\mathrm{~d}, J=8.2 \mathrm{~Hz}, 1 \mathrm{H}), 7.70(\mathrm{~d}, J=8.2 \mathrm{~Hz}, 1 \mathrm{H}), 7.40(\mathrm{~s}, 1 \mathrm{H}), 4.17(\mathrm{~s}, 3 \mathrm{H}), 3.98(\mathrm{~s}, 3 \mathrm{H}) ;{ }^{13} \mathrm{C} \mathrm{NMR}$ $\left(101 \mathrm{MHz}, \mathrm{CDCl}_{2} \mathrm{CDCl}_{2}\right) \delta 166.29,148.77,147.21,146.84,145.63,145.50,145.31,145.26$, $145.12,144.79,144.63,144.48,144.39,144.29,144.22,143.55,143.50,143.33,141.92,141.74$, $141.69,141.32,141.18,140.97,140.75,140.56,140.15,139.57,138.07,136.56,135.47,135.22$, 132.80, 126.25, 124.59, 120.08, 116.68, 109.02, 79.62, 64.86, 59.72, 51.53; FT-IR v/cm ${ }^{-1}(\mathrm{KBr})$ 2926, 2849, 1725, 1610, 1433, 1374, 1333, 1224, 1101, 1036, 998, 844, 759, 741, 527; UV-vis $\left(\mathrm{CHCl}_{3}\right) \lambda_{\max } / \mathrm{nm}(\log \varepsilon) 255$ (5.03), 317 (4.73), 418 (3.70), 476 (3.29); MALDI-TOF MS $m / z$ calcd for $\mathrm{C}_{71} \mathrm{H}_{7} \mathrm{~N}_{2} \mathrm{O}_{3}[\mathrm{M}-\mathrm{OMe}]^{-}$935.0462, found 935.0472.

\section{Synthesis and spectral data of compound $2 \mathrm{f}$}

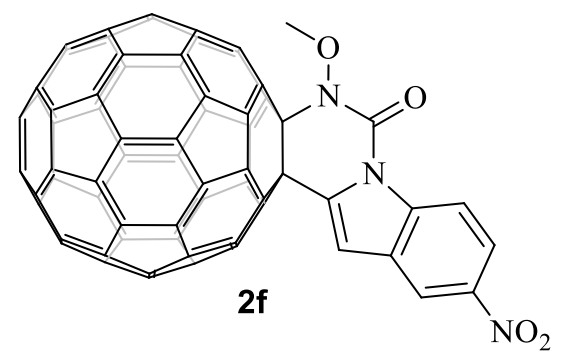

Preparation of 2f: By following the general procedure, the reaction of $\mathrm{C}_{60}(36.0 \mathrm{mg}, 0.05 \mathrm{mmol})$ with 1 f (35.0 mg, $0.15 \mathrm{mmol}), \mathrm{Pd}(\mathrm{OAc})_{2}(1.2 \mathrm{mg}, 0.005 \mathrm{mmol}), \mathrm{K}_{2} \mathrm{~S}_{2} \mathrm{O}_{8}(27.1 \mathrm{mg}, 0.10 \mathrm{mmol})$, and TFA $(0.2 \mathrm{~mL})$ in $\mathrm{ODCB}(5 \mathrm{~mL})$ at $90{ }^{\circ} \mathrm{C}$ for $28 \mathrm{~h}$ afforded recovered $\mathrm{C}_{60}(16.1 \mathrm{mg}, 45 \%)$ and 2f (11.2 mg, 24\%): amorphous brown solid; ${ }^{1} \mathrm{H}$ NMR (400 MHz, $\left.\mathrm{CDCl}_{2} \mathrm{CDCl}_{2}\right) \delta 8.74$ (d, $J=9.1$ $\mathrm{Hz}, 1 \mathrm{H}), 8.56(\mathrm{~d}, J=2.0 \mathrm{~Hz}, 1 \mathrm{H}), 8.37\left(\mathrm{dd}, J_{1}=9.1 \mathrm{~Hz}, J_{2}=2.0 \mathrm{~Hz}, 1 \mathrm{H}\right), 7.50(\mathrm{~s}, 1 \mathrm{H}), 4.18(\mathrm{~s}$, $3 \mathrm{H})$; The ${ }^{13} \mathrm{C}$ NMR spectrum of $2 \mathbf{f}$ with good $\mathrm{S} / \mathrm{N}$ ratio could not be obtained due to its low solubility. FT-IR $v / \mathrm{cm}^{-1}$ (KBr) 2923, 2849, 1731, 1522, 1454, 1442, 1377, 1333, 1263, 1030, 892, 817, 744, 527; UV-vis $\left(\mathrm{CHCl}_{3}\right) \lambda_{\max } / \mathrm{nm}(\log \varepsilon) 257$ (4.98), 317 (4.59), 415 (3.58), 475 (3.18); MALDI-TOF MS m/z calcd for $\mathrm{C}_{70} \mathrm{H}_{7} \mathrm{~N}_{3} \mathrm{O}_{4}[\mathrm{M}]^{-}$953.0442, found 953.0441.

\section{Synthesis and spectral data of compound $2 \mathrm{~g}$}




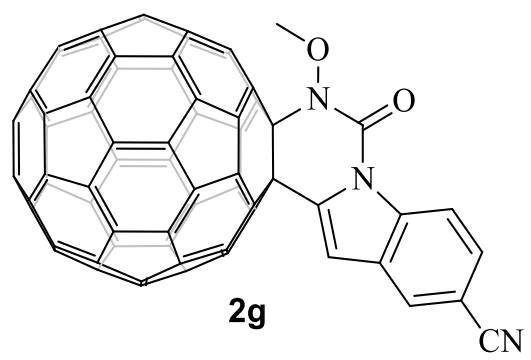

Preparation of $2 \mathrm{~g}$ : By following the general procedure, the reaction of $\mathrm{C}_{60}(36.0 \mathrm{mg}, 0.05 \mathrm{mmol})$ with $1 \mathrm{~g}(33.4,0.15 \mathrm{mmol}), \mathrm{Pd}(\mathrm{OAc})_{2}(2.2 \mathrm{mg}, 0.01 \mathrm{mmol}), \mathrm{K}_{2} \mathrm{~S}_{2} \mathrm{O}_{8}(27.1 \mathrm{mg}, 0.10 \mathrm{mmol})$, and TFA $(0.2 \mathrm{~mL})$ in ODCB $(5 \mathrm{~mL})$ at $90{ }^{\circ} \mathrm{C}$ for $48 \mathrm{~h}$ afforded recovered $\mathrm{C}_{60}(18.5 \mathrm{mg}, 51 \%)$ and $\mathbf{2 g}$ (12.8 mg, 28\%): amorphous brown solid; ${ }^{1} \mathrm{H}$ NMR (400 MHz, $\left.\mathrm{CDCl}_{2} \mathrm{CDCl}_{2}\right) \delta 8.72(\mathrm{~d}, J=8.6$ $\mathrm{Hz}, 1 \mathrm{H}), 7.98(\mathrm{~s}, 1 \mathrm{H}), 7.75\left(\mathrm{dd}, J_{1}=8.6 \mathrm{~Hz}, J_{2}=1.3 \mathrm{~Hz}, 1 \mathrm{H}\right), 7.42(\mathrm{~s}, 1 \mathrm{H}), 4.17(\mathrm{~s}, 3 \mathrm{H}) ;{ }^{13} \mathrm{C} \mathrm{NMR}$ $\left(101 \mathrm{MHz}, \mathrm{CDCl}_{2} \mathrm{CDCl}_{2}\right) \delta 148.51,147.27,146.89,145.69,145.55,145.37,145.31,145.18$, $144,63,144.59,144.50,144.47,144.35,144.27,143.58,143.53,143.21,141.97,141.81,141.75$, 141.36, 141.22, 140.96, 140.79, 140.61, 140.16, 139.64, 138.11, 137.75, 136.07, 135.19, 129.16, 127.78, 124.92, 118.64, 116.13, 108.57, 106.61, 79.78, 64.88, 59.50; FT-IR $v / \mathrm{cm}^{-1}(\mathrm{KBr}) 2917$, 2849, 2228, 1739, 1636, 1463, 1377, 1260, 1180, 1115, 1033, 992, 880, 815, 803, 744, 530; UVvis $\left(\mathrm{CHCl}_{3}\right) \lambda_{\text {max }} / \mathrm{nm}(\log \varepsilon) 256$ (5.02), 317 (4.53), 415 (3.57), 477 (3.15); MALDI-TOF MS m/z calcd for $\mathrm{C}_{70} \mathrm{H}_{4} \mathrm{~N}_{3} \mathrm{O}$ [M-OMe] 902.0360 , found 902.0368 .

\section{Synthesis and spectral data of compound $2 \mathrm{~h}$}

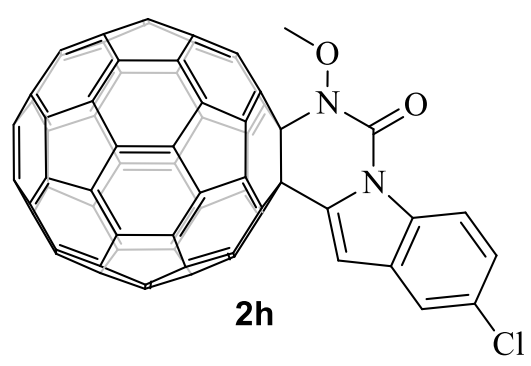

Preparation of $\mathbf{2 h}$ : By following the general procedure, the reaction of $\mathrm{C}_{60}(36.1 \mathrm{mg}, 0.05 \mathrm{mmol})$ with $1 \mathrm{~h}$ (35.5 mg, $0.15 \mathrm{mmol}), \mathrm{Pd}(\mathrm{OAc})_{2}(1.2 \mathrm{mg}, 0.005 \mathrm{mmol}), \mathrm{K}_{2} \mathrm{~S}_{2} \mathrm{O}_{8}(27.1 \mathrm{mg}, 0.10 \mathrm{mmol})$, and TFA $(0.2 \mathrm{~mL})$ in ODCB $(5 \mathrm{~mL})$ at $100^{\circ} \mathrm{C}$ for $36 \mathrm{~h}$ afforded recovered $\mathrm{C}_{60}(19.8 \mathrm{mg}, 55 \%)$ and 2h (15.1 mg, 32\%): amorphous brown solid; ${ }^{1} \mathrm{H}$ NMR (400 MHz, $\left.\mathrm{CDCl}_{2} \mathrm{CDCl}_{2}\right) \delta 8.51$ (d, $J=8.8$ $\mathrm{Hz}, 1 \mathrm{H}), 7.62(\mathrm{~d}, J=1.9 \mathrm{~Hz}, 1 \mathrm{H}), 7.46\left(\mathrm{dd}, J_{1}=8.8 \mathrm{~Hz}, J_{2}=1.9 \mathrm{~Hz}, 1 \mathrm{H}\right), 7.29(\mathrm{~s}, 1 \mathrm{H}), 4.15$ (s, $3 \mathrm{H}) ;{ }^{13} \mathrm{C} \mathrm{NMR}\left(101 \mathrm{MHz}, \mathrm{CDCl}_{2} \mathrm{CDCl}_{2}\right) \delta 148.90,147.24,146.86,145.65,145.51,145.33,145.27$, $145.14,144.92$, 144.68, 144.51, 144.40, 144.32, 144.23, 143.58, 143.52, 143.36, 141.93, 141.76, 
141.70, 141.34, 141.20, 141.02, 140.77, 140.58, 140.17, 139.59, 138.06, 135.26, 135.01, 134.40, 130.31, 128.98, 124.93, 119.86, 116.20, 108.42, 79.72, 64.84, 59.68; FT-IR $v / \mathrm{cm}^{-1}$ (KBr) 2923, 2852, 1733, 1651, 1610, 1580, 1545, 1448, 1371, 1268, 1142, 1068, 1042, 995, 915, 842, 812, 756, 591, 527; UV-vis $\left(\mathrm{CHCl}_{3}\right) \lambda_{\max } / \mathrm{nm}(\log \varepsilon) 256$ (5.00), 318 (4.56), 415 (3.64), 460 (3.41); MALDITOF MS $m / z$ calcd for $\mathrm{C}_{70} \mathrm{H}_{7}^{35} \mathrm{ClN}_{2} \mathrm{O}_{2}[\mathrm{M}]^{-}$942.0202, found 942.0203 .

\section{Synthesis and spectral data of compound $2 i$}

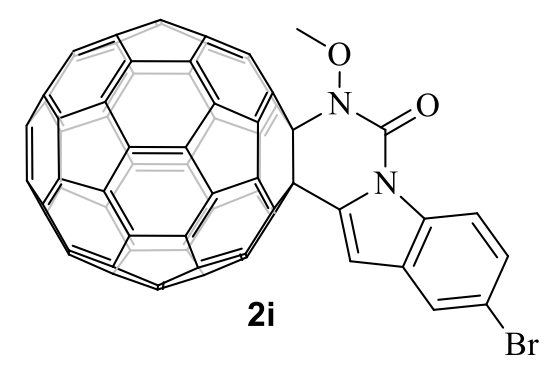

Preparation of 2i: By following the general procedure, the reaction of $\mathrm{C}_{60}(36.0 \mathrm{mg}, 0.05 \mathrm{mmol})$ with $1 \mathbf{i}$ (40.5 mg, $0.15 \mathrm{mmol}), \mathrm{Pd}(\mathrm{OAc})_{2}(1.2 \mathrm{mg}, 0.005 \mathrm{mmol}), \mathrm{K}_{2} \mathrm{~S}_{2} \mathrm{O}_{8}(27.0 \mathrm{mg}, 0.10 \mathrm{mmol})$, and TFA $(0.2 \mathrm{~mL})$ in ODCB $(5 \mathrm{~mL})$ at $100{ }^{\circ} \mathrm{C}$ for $36 \mathrm{~h}$ afforded recovered $\mathrm{C}_{60}(13.0 \mathrm{mg}, 36 \%)$ and $2 \mathbf{i}$ (13.3 mg, 27\%): amorphous brown solid; ${ }^{1} \mathrm{H} \mathrm{NMR}\left(400 \mathrm{MHz}, \mathrm{CS}_{2} / \mathrm{CDCl}_{2} \mathrm{CDCl}_{2}, 1: 2\right) \delta 8.47$ $(\mathrm{d}, J=8.8 \mathrm{~Hz}, 1 \mathrm{H}), 7.79(\mathrm{~d}, J=1.9 \mathrm{~Hz}, 1 \mathrm{H}), 7.60\left(\mathrm{dd}, J_{1}=8.8 \mathrm{~Hz}, J_{2}=1.9 \mathrm{~Hz}, 1 \mathrm{H}\right), 7.29(\mathrm{~s}, 1 \mathrm{H})$, 4.15 (s, 3H); ${ }^{13} \mathrm{C}$ NMR (101 MHz, $\left.\mathrm{CS}_{2} / \mathrm{CDCl}_{2} \mathrm{CDCl}_{2}, 1: 2\right) \delta 148.69,147.24,146.87,145.68$, 145.54, 145.36, 145.30, 145.17, 145.01, 144.75, 144.53, 144.44, 144.34, 144.26, 143.61, 143.57, $143.37,141.98,141.82,141.76,141.39,141.24,141.09,140.82$, 140.63, 140.23, 139.62, 138.11, $135.26,134.75,134.68,130.79,127.67,122.91,117.00,116.54,108.32,79.71,64.56,59.67$; FTIR $v / \mathrm{cm}^{-1}(\mathrm{KBr}) 2923,2843,1733,1442,1363,1274,1142,1056,1030,996,906,868,844,812$, 527; UV-vis $\left(\mathrm{CHCl}_{3}\right) \lambda_{\max } / \mathrm{nm}(\log \varepsilon) 255$ (5.00), 315 (3.51), 423 (3.59), 477 (3.13); MALDI-TOF MS $m / z$ calcd for $\mathrm{C}_{70} \mathrm{H}_{7}{ }^{79} \mathrm{BrN}_{2} \mathrm{O}_{2}[\mathrm{M}]^{-}$985.9696, found 985.9697.

\section{Synthesis and spectral data of compound $2 \mathrm{j}$}




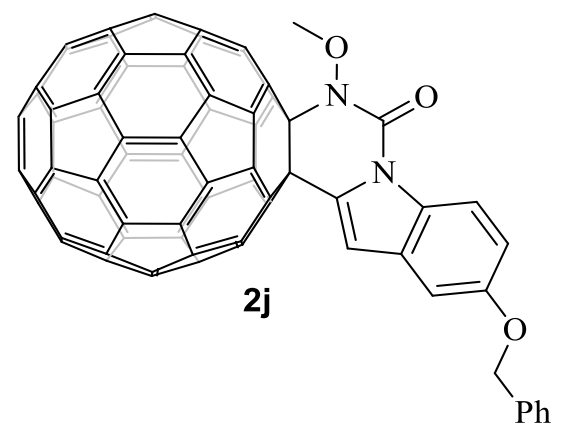

Preparation of $\mathbf{2 j}$ : By following the general procedure, the reaction of $\mathrm{C}_{60}(36.0 \mathrm{mg}, 0.05 \mathrm{mmol})$ with $1 \mathbf{j}$ (44.4 mg, $0.15 \mathrm{mmol}), \mathrm{Pd}(\mathrm{OAc})_{2}(2.2 \mathrm{mg}, 0.01 \mathrm{mmol}), \mathrm{K}_{2} \mathrm{~S}_{2} \mathrm{O}_{8}(54.0 \mathrm{mg}, 0.20 \mathrm{mmol})$, and TFA $(0.2 \mathrm{~mL})$ in $\mathrm{ODCB}(5 \mathrm{~mL})$ at $100{ }^{\circ} \mathrm{C}$ for $48 \mathrm{~h}$ afforded recovered $\mathrm{C}_{60}(20.3 \mathrm{mg}, 56 \%)$ and $\mathbf{2 j}$ (10.3 mg, 22\%): amorphous brown solid; ${ }^{1} \mathrm{H}$ NMR (400 MHz, $\left.\mathrm{CDCl}_{2} \mathrm{CDCl}_{2}\right) \delta 8.46(\mathrm{~d}, J=8.7 \mathrm{~Hz}$, $1 \mathrm{H}), 7.45(\mathrm{~d}, J=7.1 \mathrm{~Hz}, 2 \mathrm{H}), 7.37$ (d, $J=7.4 \mathrm{~Hz}, 2 \mathrm{H}), 7.31$ (t, $J=7.3 \mathrm{~Hz}, 1 \mathrm{H}), 7.27$ (s, 1H), $7.22-$ $7.17(\mathrm{~m}, 2 \mathrm{H}), 5.11(\mathrm{~s}, 2 \mathrm{H}), 4.14(\mathrm{~s}, 3 \mathrm{H}) ;{ }^{13} \mathrm{C}$ NMR $\left(101 \mathrm{MHz}, \mathrm{CDCl}_{2} \mathrm{CDCl}_{2}\right) \delta 155.12,149.22$, 147.21, 146.83, 145.62, 145.47, 145.30, 145.24, 145.11, 144.75, 144.53, 144.34, 144.30, 144.20, $143.59,143.52,141.91,141.73,141.68,141.32,141.19,141.08,140.76,140.56,140.19,139.54$, 138.02, 135.90, 135.31, 134.20, 130.89, 130.02, 127.68, 127.10, , 126.55, 115.91, 114.23, 109.17, 104.33, 79.63, 69.61, 64.83, 59.81; FT-IR $v / \mathrm{cm}^{-1}$ (KBr) 2920, 2846, 1722, 1610, 1510, 1466, 1445, 1380, 1189, 1033, 995, 847, 812, 733, 694, 530; UV-vis $\left(\mathrm{CHCl}_{3}\right) \lambda_{\max } / \mathrm{nm}(\log \varepsilon) 256$ (5.00), 315 (4.55), 405 (3.72), 465 (3.37); MALDI-TOF MS $m / z$ calcd for $\mathrm{C}_{77} \mathrm{H}_{14} \mathrm{~N}_{2} \mathrm{O}_{3}[\mathrm{M}]^{-} 1014.1010$, found 1014.1021.

\section{Synthesis and spectral data of compound $2 \mathrm{k}$}

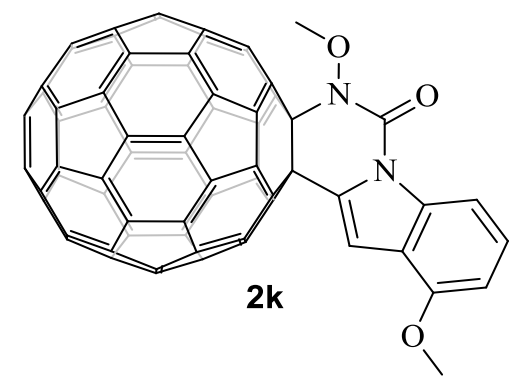

Preparation of $2 \mathrm{k}$ : By following the general procedure, the reaction of $\mathrm{C}_{60}(35.9 \mathrm{mg}, 0.05 \mathrm{mmol})$ with $1 \mathrm{k}(33.0 \mathrm{mg}, 0.15 \mathrm{mmol}), \mathrm{Pd}(\mathrm{OAc})_{2}(2.1 \mathrm{mg}, 0.01 \mathrm{mmol}), \mathrm{K}_{2} \mathrm{~S}_{2} \mathrm{O}_{8}(54.2 \mathrm{mg}, 0.20 \mathrm{mmol})$, and TFA $(0.2 \mathrm{~mL})$ in ODCB $(5 \mathrm{~mL})$ at $100{ }^{\circ} \mathrm{C}$ for $48 \mathrm{~h}$ afforded recovered $\mathrm{C}_{60}(18.3 \mathrm{mg}, 51 \%)$ and $\mathbf{2 k}$ (15.4 mg, 33\%): amorphous brown solid; ${ }^{1} \mathrm{H} \mathrm{NMR}\left(400 \mathrm{MHz}, \mathrm{CDCl}_{2} \mathrm{CDCl}_{2}\right) \delta 8.17$ (d, $J=8.3 \mathrm{~Hz}$, 
1H), $7.45(\mathrm{t}, J=8.2 \mathrm{~Hz}, 1 \mathrm{H}), 7.44(\mathrm{~s}, 1 \mathrm{H}), 6.82(\mathrm{~d}, J=8.1 \mathrm{~Hz}, 1 \mathrm{H}), 4.13(\mathrm{~s}, 3 \mathrm{H}), 3.93(\mathrm{~s}, 3 \mathrm{H}) ;{ }^{13} \mathrm{C}$ NMR (101 MHz, $\left.\mathrm{CDCl}_{2} \mathrm{CDCl}_{2}\right) \delta 152.09,149.44,147.22,146.83,145.63,145.46,145.30,145.24$, $145.11,144.75,144.54,144.34,144.29,144.19,143.62,143.51,141.89,141.73,141.67,141.32$, $141.21,141.12,140.75,140.58,140.19,139.53,138.00,137.22,135.36,131.98,126.13,119.39$, 107.90, 106.55, 103.81, 79.69, 64.77, 59.77, 54.66; FT-IR v/cm ${ }^{-1}$ (KBr) 2923, 2855, 1725, 1610, $1430,1368,1336,1298,1227,1101,1039,995,842,762,744,527 ; \mathrm{UV}$-vis $\left(\mathrm{CHCl}_{3}\right) \lambda_{\max } / \mathrm{nm}(\log$ ع) 256 (5.06), 317 (4.75), 429 (3.60), 477 (3.22); MALDI-TOF MS $m / z$ calcd for $\mathrm{C}_{71} \mathrm{H}_{10} \mathrm{~N}_{2} \mathrm{O}_{3}$ [M] 938.0697, found 938.0688.

\section{Synthesis and spectral data of compound 21}

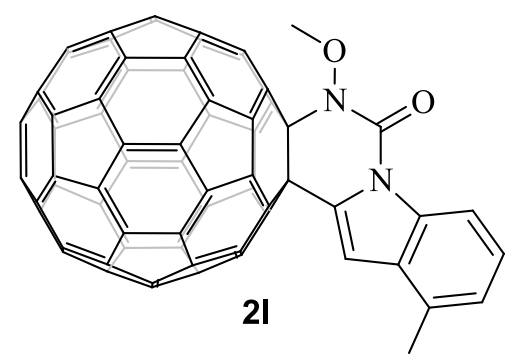

Preparation of 21: By following the general procedure, the reaction of $\mathrm{C}_{60}(35.9 \mathrm{mg}, 0.05 \mathrm{mmol})$ with 11 (30.8 mg, $0.15 \mathrm{mmol}), \mathrm{Pd}(\mathrm{OAc})_{2}(2.2 \mathrm{mg}, 0.01 \mathrm{mmol}), \mathrm{K}_{2} \mathrm{~S}_{2} \mathrm{O}_{8}$ (40.4 mg, $0.15 \mathrm{mmol}$ ), and TFA $(0.2 \mathrm{~mL})$ in ODCB $(5 \mathrm{~mL})$ at $100{ }^{\circ} \mathrm{C}$ for $36 \mathrm{~h}$ afforded recovered $\mathrm{C}_{60}(20.0 \mathrm{mg}, 56 \%)$ and $2 \mathrm{l}$ (14.0 mg, 31\%): amorphous brown solid; ${ }^{1} \mathrm{H} \mathrm{NMR}\left(400 \mathrm{MHz}, \mathrm{CDCl}_{2} \mathrm{CDCl}_{2}\right) \delta 8.40(\mathrm{~d}, J=8.2 \mathrm{~Hz}$, $1 \mathrm{H}), 7.41(\mathrm{t}, J=7.9 \mathrm{~Hz} 1 \mathrm{H}), 7.32(\mathrm{~s}, 1 \mathrm{H}), 7.19(\mathrm{~d}, J=7.4 \mathrm{~Hz}, 1 \mathrm{H}), 4.13(\mathrm{~s}, 3 \mathrm{H}), 2.55(\mathrm{~s}, 3 \mathrm{H}) ;{ }^{13} \mathrm{C}$ $\operatorname{NMR}\left(101 \mathrm{MHz}, \mathrm{CDCl}_{2} \mathrm{CDCl}_{2}\right) \delta 149.45,147.21,146.84,145.63,145.47,145.30,145.24,145.20$, $145.11,144.78,144.53,144.35,144.30,144.20,143.60,143.53,141.90,141.74,141.68,141.33$, $141.20,141.12,140.77,140.58,140.20,139.58,138.02,136.00,135.35,133.08,130.11,128.84$, 125.13, 123.98, 112.57, 107.88, 79.70, 64.76, 59.90, 17.74; FT-IR v/cm-1 (KBr) 2920, 2852, 1716, $1554,1510,1418,1357,1330,1277,1030,842,765,577,527 ; \mathrm{UV}$-vis $\left(\mathrm{CHCl}_{3}\right) \lambda_{\max } / \mathrm{nm}(\log \varepsilon)$ 255 (5.02), 313 (4.61), 409 (3.77), 467 (3.49); MALDI-TOF MS m/z calcd for $\mathrm{C}_{71} \mathrm{H}_{10} \mathrm{~N}_{2} \mathrm{O}_{2}$ [M] ${ }^{-}$ 922.0748, found 922.0762.

\section{Synthesis and spectral data of compound $2 \mathrm{~m}$}




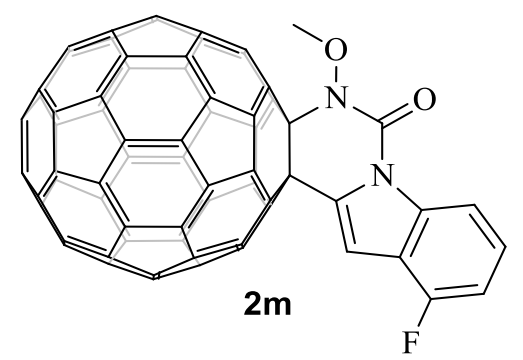

Preparation of $\mathbf{2 m}$ : By following the general procedure, the reaction of $\mathrm{C}_{60}(36.0 \mathrm{mg}, 0.05 \mathrm{mmol})$ with $1 \mathrm{~m}$ (31.6 mg, $0.15 \mathrm{mmol}), \mathrm{Pd}(\mathrm{OAc})_{2}(1.1 \mathrm{mg}, 0.005 \mathrm{mmol}), \mathrm{K}_{2} \mathrm{~S}_{2} \mathrm{O}_{8}(27.0 \mathrm{mg}, 0.10 \mathrm{mmol})$, and TFA $(0.2 \mathrm{~mL})$ in ODCB $(5 \mathrm{~mL})$ at $100{ }^{\circ} \mathrm{C}$ for $28 \mathrm{~h}$ afforded recovered $\mathrm{C}_{60}(18.2 \mathrm{mg}, 50 \%)$ and 2m (14.7 mg, 32\%): amorphous brown solid; ${ }^{1} \mathrm{H} \mathrm{NMR}\left(400 \mathrm{MHz}, \mathrm{CS}_{2} / \mathrm{CDCl}_{2} \mathrm{CDCl}_{2}, 3: 1\right) \delta 8.35$ (d, $J=8.3 \mathrm{~Hz}, 1 \mathrm{H}), 7.47-7.41$ (m, 1H), 7.42 (s, 1H), 7.04 (t, $J=8.7 \mathrm{~Hz}, 1 \mathrm{H}), 4.17$ (s, 3H); ${ }^{13} \mathrm{C}$ NMR (101 MHz, $\left.\mathrm{CS}_{2} / \mathrm{CDCl}_{2} \mathrm{CDCl}_{2}, 3: 1\right) \delta 154.70$ (d, $\left.J=250.4 \mathrm{~Hz}\right), 148.68,147.22,146.84$, 145.67, 145.52, 145.34, 145.29, 145.15, 145.05, 144.80, 144.51, 144.47, 144.32, 144.24, 143.63, 143.56, 141.97, 141.81, 141.75, 141.37, 141.25, 141.10, 140.81, 140.66, 140.24, 139.64, 138.09, $137.91(\mathrm{~d}, J=8.9 \mathrm{~Hz}), 135.26,133.35,125.82(\mathrm{~d}, J=7.0 \mathrm{~Hz}), 118.09$ (d, $J=22.1 \mathrm{~Hz}), 111.23(\mathrm{~d}$, $J=3.9 \mathrm{~Hz}), 108.74$ (d, $J=18.2 \mathrm{~Hz}), 104.80,79.63,64.30,59.62$; FT-IR $v / \mathrm{cm}^{-1}(\mathrm{KBr}) 2923,2852$, $1713,1628,1489,1430,1377,1265,1236,1101,1015,977,871,842,768,524$; UV-vis $\left(\mathrm{CHCl}_{3}\right)$ $\lambda_{\max } / \mathrm{nm}(\log \varepsilon) 254$ (5.08), 316 (4.64), 418 (3.80), 454 (3.62); MALDI-TOF MS $m / z$ calcd for $\mathrm{C}_{70} \mathrm{H}_{7} \mathrm{FN}_{2} \mathrm{O}_{2}[\mathrm{M}]^{-}$926.0497, found 926.0488 .

\section{Synthesis and spectral data of compound $2 n$}

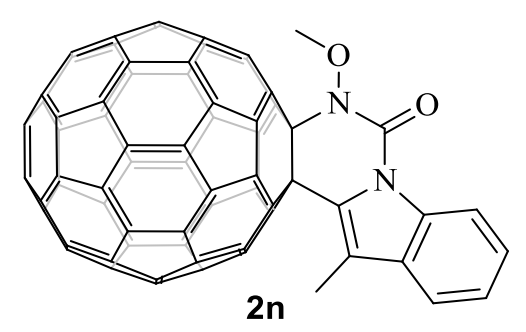

Preparation of $2 \mathrm{n}$ : By following the general procedure, the reaction of $\mathrm{C}_{60}(36.1 \mathrm{mg}, 0.05 \mathrm{mmol})$ with $1 \mathrm{n}$ (30.7 mg, $0.15 \mathrm{mmol}), \mathrm{Pd}(\mathrm{OAc})_{2}(1.2 \mathrm{mg}, 0.005 \mathrm{mmol}), \mathrm{K}_{2} \mathrm{~S}_{2} \mathrm{O}_{8}(27.1 \mathrm{mg}, 0.10 \mathrm{mmol})$, and TFA $(0.2 \mathrm{~mL})$ in ODCB $(5 \mathrm{~mL})$ at $100{ }^{\circ} \mathrm{C}$ for $28 \mathrm{~h}$ afforded recovered $\mathrm{C}_{60}(22.1 \mathrm{mg}, 61 \%)$ and 2n (9.2 mg, 20\%): amorphous brown solid; ${ }^{1} \mathrm{H} \mathrm{NMR}\left(400 \mathrm{MHz}, \mathrm{CS}_{2} / \mathrm{CDCl}_{2} \mathrm{CDCl}_{2}, 2: 3\right) \delta 8.51$ (d, $J=8.1 \mathrm{~Hz}, 1 \mathrm{H}), 7.64(\mathrm{~d}, J=7.6 \mathrm{~Hz}, 1 \mathrm{H}), 7.52\left(\mathrm{td}, J_{1}=7.4 \mathrm{~Hz}, J_{2}=1.2 \mathrm{~Hz}, 1 \mathrm{H}\right), 7.41\left(\mathrm{td}, J_{1}=7.7\right.$ $\left.\mathrm{Hz}, J_{2}=1.0 \mathrm{~Hz}, 1 \mathrm{H}\right), 4.08(\mathrm{~s}, 3 \mathrm{H}), 2.58(\mathrm{~s}, 3 \mathrm{H})$; The ${ }^{13} \mathrm{C}$ NMR spectrum of $2 \mathbf{n}$ with good $\mathrm{S} / \mathrm{N}$ ratio 
could not be obtained due to its low solubility. FT-IR $v / \mathrm{cm}^{-1}(\mathrm{KBr}) 2920,2846,1719,1516,1418$, 1380, 1330, 1027, 877, 842, 794, 768, 530; UV-vis $\left(\mathrm{CHCl}_{3}\right) \lambda_{\max } / \mathrm{nm}(\log \varepsilon) 256$ (5.00), 315 (4.56), 417 (3.60), 460 (3.34); MALDI-TOF MS m/z calcd for $\mathrm{C}_{71} \mathrm{H}_{10} \mathrm{~N}_{2} \mathrm{O}_{2}$ [M] 922.0748, found 922.0749 .

\section{Synthesis and spectral data of compounds 3a-d} Synthesis and spectral data of compound $3 a$

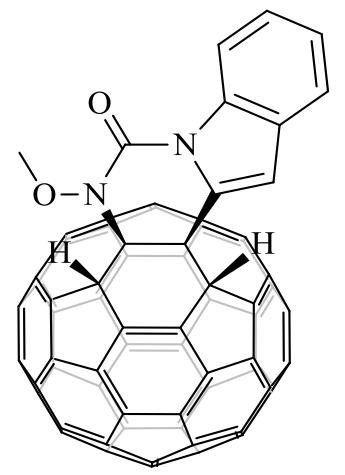

$3 a$

$20.0 \mathrm{mg}(0.022 \mathrm{mmol})$ of $\mathbf{2 a}$ was electroreduced by controlled potential electrolysis (CPE) at $1.14 \mathrm{~V}$ vs saturated calomel electrode (SCE) in $30 \mathrm{~mL}$ of ODCB containing $0.1 \mathrm{M} n$ butylammonium perchlorate (TBAP) under argon atmosphere at room temperature. The electrolysis was terminated when the theoretical number of coulombs required for a full conversion of $\mathbf{2 a}$ to $2 \mathbf{a}^{2-}$ was reached. Then, the dianionic $2 \mathbf{a}$ was reacted with TFA (5 $\left.\mu \mathrm{L}, 0.066 \mathrm{mmol}\right)$ at ambient temperature for $15 \mathrm{~min}$. The reaction mixture was filtered through a silica gel (200-300 mesh) plug with $\mathrm{CS}_{2} / \mathrm{CH}_{2} \mathrm{Cl}_{2}(1: 1, \mathrm{v} / \mathrm{v})$ to remove the electrolyte. After evaporation in vacuo, the residue was separated by silica gel (400-500 mesh) column with $\mathrm{CS}_{2} / \mathrm{CH}_{2} \mathrm{Cl}_{2}$ (4:1, v/v) to afford 3a (17.3 mg, 79\%) as an amorphous brown solid. ${ }^{1} \mathrm{H}$ NMR (400 MHz, $\mathrm{CS}_{2}$ with DMSO- $d_{6}$ as the external deuterium lock and containing TMS as the reference) $\delta 8.41(\mathrm{~d}, J=8.5 \mathrm{~Hz}, 1 \mathrm{H}), 7.63(\mathrm{~d}$, $J=7.2 \mathrm{~Hz}, 1 \mathrm{H}), 7.40\left(\mathrm{ddd}, J_{1}=7.6 \mathrm{~Hz}, J_{2}=7.2 \mathrm{~Hz}, J_{3}=1.1 \mathrm{~Hz}, 1 \mathrm{H}\right), 7.31\left(\mathrm{ddd}, J_{1}=8.5 \mathrm{~Hz}, J_{2}=\right.$ $\left.7.6 \mathrm{~Hz}, J_{3}=1.1 \mathrm{~Hz}, 1 \mathrm{H}\right), 7.27(\mathrm{~s}, 1 \mathrm{H}), 6.80(\mathrm{~d}, J=1.6 \mathrm{~Hz}, 1 \mathrm{H}), 6.46(\mathrm{~d}, J=1.6 \mathrm{~Hz}, 1 \mathrm{H}), 4.36(\mathrm{~s}$, $3 \mathrm{H}) ;{ }^{13} \mathrm{C}$ NMR $\left(101 \mathrm{MHz}, \mathrm{CS}_{2}\right.$ with DMSO- $d_{6}$ as the external deuterium lock and containing TMS as the reference) $\delta 149.84,149.06,148.22,148.14,147.69,147.41,147.31,147.05,146.86,145.97$, $145.95,145.93,145.58,145.48,145.40,145.37,145.00,144.85,144.46,144.04,143.91,143.85$, $143.83,143.76,143.69,143.51,143.26,143.18,143.13,143.04,142.95,142.93,142.81,142.61$, 
$142.27,142.11,142.06,141.75,141.73,141.70,141.46,141.43,141.24,140.93,140.66,140.63$, $140.18,139.85,139.82$, 138.06, 137.98, 135.86, 135.52, 134.37, 129.05, 124.72, 124.30, 120.01, 115.04, 105.52, 76.69, 63.75, 59.66, 56.24, 55.57; FT-IR v/cm ${ }^{-1}$ (KBr) 2920, 2846, 1719, 1657 , 1451, 1363, 1265, 1095, 1018, 877, 803, 744, 527; UV-vis $\left(\mathrm{CHCl}_{3}\right) \lambda_{\max } / \mathrm{nm}(\log \varepsilon) 255(5.00)$, 296 (4.68), 331 (4.46), 429 (3.85); MALDI-TOF MS m/z calcd for $\mathrm{C}_{70} \mathrm{H}_{10} \mathrm{~N}_{2} \mathrm{O}_{2}[\mathrm{M}]^{-} 910.0748$, found 910.0749 .

\section{Synthesis and spectral data of compound $3 \mathrm{~b}$}

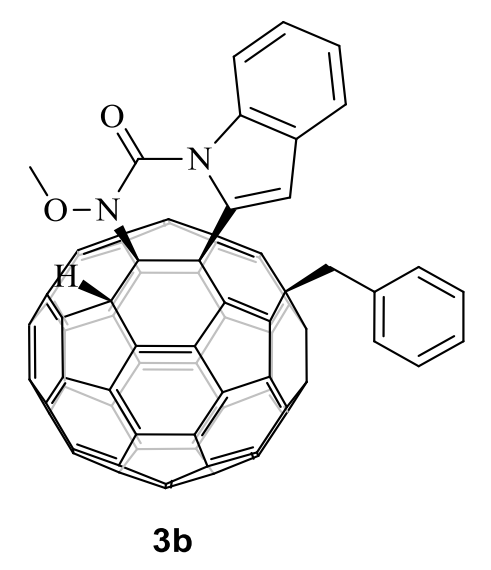

$20.0 \mathrm{mg}(0.022 \mathrm{mmol})$ of $2 \mathrm{a}$ was electroreduced by CPE at $-1.14 \mathrm{~V}$ vs SCE in $25 \mathrm{~mL}$ of ODCB containing 0.1 M TBAP under argon atmosphere at room temperature. The electrolysis was terminated when the theoretical number of coulombs required for a full conversion of $\mathbf{2 a}$ to $\mathbf{2} \mathbf{a}^{2-}$ was reached. Then, the dianionic $2 \mathbf{a}$ was reacted with benzyl bromide $(8 \mu \mathrm{L}, 0.066 \mathrm{mmol})$ for $5 \mathrm{~h}$ and then TFA $(5 \mu \mathrm{L}, 0.066 \mathrm{mmol})$ at room temperature for $30 \mathrm{~min}$. The reaction mixture was filtered through a silica gel (200-300 mesh) plug with $\mathrm{CS}_{2} / \mathrm{CH}_{2} \mathrm{Cl}_{2}(1: 1, \mathrm{v} / \mathrm{v})$ to remove the electrolyte. After evaporation in vacuo, the residue was separated by silica gel (400-500 mesh) column with $\mathrm{CS}_{2} / \mathrm{CH}_{2} \mathrm{Cl}_{2}(4: 1, \mathrm{v} / \mathrm{v})$ to afford $3 \mathbf{b}(16.4 \mathrm{mg}, 75 \%)$ as an amorphous brown solid and recovered $2 \mathrm{a}(1.2 \mathrm{mg}, 6 \%) .{ }^{1} \mathrm{H}$ NMR $\left(400 \mathrm{MHz}, \mathrm{CS}_{2}\right.$ with DMSO- $d_{6}$ as the external deuterium lock and containing TMS as the reference) $\delta 8.44(\mathrm{~d}, J=8.2 \mathrm{~Hz}, 1 \mathrm{H}), 7.56(\mathrm{~d}, J=7.8 \mathrm{~Hz}, 1 \mathrm{H})$, $7.38\left(\mathrm{td}, J_{1}=7.4 \mathrm{~Hz}, J_{2}=0.9 \mathrm{~Hz}, 1 \mathrm{H}\right), 7.34-7.25(\mathrm{~m}, 3 \mathrm{H}), 7.24-7.15(\mathrm{~m}, 3 \mathrm{H}), 7.10(\mathrm{~s}, 1 \mathrm{H}), 6.52$ (s, 1H), 4.32 (s, 3H), 4.24 (d, $J=12.8 \mathrm{~Hz} 1 \mathrm{H}), 4.13(\mathrm{~d}, J=12.8 \mathrm{~Hz}, 1 \mathrm{H}) ;{ }^{13} \mathrm{C}$ NMR $(101 \mathrm{MHz}$, $\mathrm{CS}_{2}$ with DMSO- $d_{6}$ as the external deuterium lock and reference) $\delta 157.76,157.22,151.29,150.41$, $148.90,148.47,148.28,147.88,147.33,147.26,147.01,146.89,146.82,146.72,146.03,145.65$, $145.38,145.18,145.17,144.79,144.74,144.61,144.57,144.47,144.30,144.20,144.16,143.93$, 
$143.80,143.77,143.62,143.56,143.50,143.23,143.20,143.15,143.02,142.93,142.22,141.99$, 141.30, 141.02, 140.68, 140.42, 140.39, 140.06, 140.04, 140.00, 139.81, 139.51, 139.08, 135.32, 135.04, 134.59, 133.77, 129.63, 129.07, 128.64, 127.10, 126.10, 124.45, 123.20, 119.76, 115.29, 105.03, 71.56, 62.96, 58.46, 58.33, 57.21, 44.92; FT-IR v/cm ${ }^{-1}$ (KBr) 2923, 2849, 1722, 1489 , 1445, 1363, 1330, 1260, 1121, 1036, 1018, 839, 744, 697, 524; UV-vis $\left(\mathrm{CHCl}_{3}\right) \lambda_{\max } / \mathrm{nm}(\log \varepsilon)$ 246 (4.98), 327 (4.74), 435 (3.95); MALDI-TOF MS $m / z$ calcd for $\mathrm{C}_{77} \mathrm{H}_{16} \mathrm{~N}_{2} \mathrm{O}_{2}$ [M] $]^{-} 1000.1217$, found 1000.1201 .

\section{Synthesis and spectral data of compound 3c}

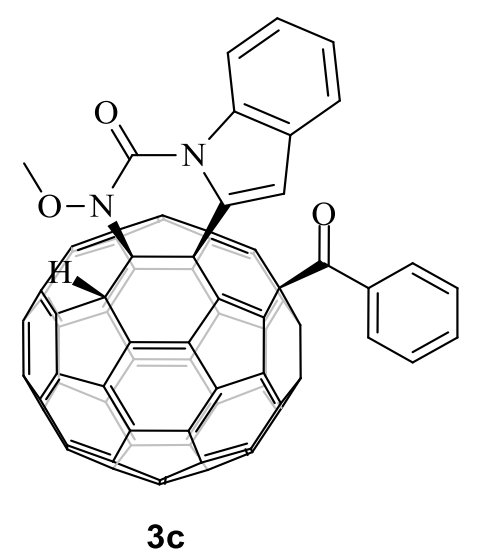

$20.0 \mathrm{mg}(0.022 \mathrm{mmol})$ of $\mathbf{2 a}$ was electroreduced by CPE at $-1.14 \mathrm{~V}$ vs SCE in $25 \mathrm{~mL}$ of ODCB containing 0.1 M TBAP under argon atmosphere at room temperature. The electrolysis was terminated when the theoretical number of coulombs required for a full conversion of $\mathbf{2 a}$ to $\mathbf{2 a}^{2-}$ was reached. Then, the dianionic $2 \mathbf{a}$ was reacted with benzoyl chloride ( $26 \mu \mathrm{L}, 0.22 \mathrm{mmol})$ in the presence of $\mathrm{NaH}(57-63 \%$ oil dispersion, $17.6 \mathrm{mg}, 0.44 \mathrm{mmol})$ at $0{ }^{\circ} \mathrm{C}$ for $24 \mathrm{~h}$ and then TFA $(5$ $\mathrm{uL}, 0.066 \mathrm{mmol})$ at $0{ }^{\circ} \mathrm{C}$ for $30 \mathrm{~min}$. The reaction mixture was filtered through a silica gel (200300 mesh) plug with $\mathrm{CS}_{2} / \mathrm{CH}_{2} \mathrm{Cl}_{2}(1: 1, \mathrm{v} / \mathrm{v})$ to remove the electrolyte. After evaporation in vacuo, the residue was separated by silica gel (400-500 mesh) column with $\mathrm{CS}_{2} / \mathrm{CH}_{2} \mathrm{Cl}_{2}$ (4:1, v/v) to afford 3c (9.2 mg, 41\%) as an amorphous brown solid and recovered $2 \mathbf{a}(2.1 \mathrm{mg}, 11 \%)$. ${ }^{1} \mathrm{H} \mathrm{NMR}$ $\left(400 \mathrm{MHz}, \mathrm{CDCl}_{2} \mathrm{CDCl}_{2}\right) \delta 8.39(\mathrm{~d}, J=8.2 \mathrm{~Hz}, 1 \mathrm{H}), 8.32(\mathrm{~d}, J=7.4 \mathrm{~Hz}, 2 \mathrm{H}), 7.61$ (t, $J=7.2 \mathrm{~Hz}$, 1H), 7.56-7.48 (m, 4H), 7.43 (t, $J=7.5 \mathrm{~Hz}, 1 \mathrm{H}), 7.33$ (t, $J=7.5 \mathrm{~Hz}, 1 \mathrm{H}), 6.74(\mathrm{~s}, 1 \mathrm{H}), 4.24$ (s, $3 \mathrm{H}) ;{ }^{13} \mathrm{C} \mathrm{NMR}\left(101 \mathrm{MHz}, \mathrm{CDCl}_{2} \mathrm{CDCl}_{2}\right) \delta 194.76,152.40,151.51,150.31,149.84,148.95,148.00$, $147.51,147.43,147.36,147.18,146.90,146.78,146.40,146.22,146.13,146.07,145.86,145.68$, $145.24,145.04,145.00,144.92,144.62,144.56,144.37,144.27,144.00,143.87,143.78,143.74$, 
$143.71,143.43,143.26,143.10,143.02,142.80,141.75,141.71,141.43,141.36,141.00,140.99$, $140.74,140.66,140.53,140.40,140.33$, 139.32, 139.28, 139.12, 137.23, 136.21, 135.96, 134.92, $134.04,132.78,128.58,128.06,128.02,124.78,123.32,120.34,114.57,106.83,77.70,68.37$, 64.65, 55.52, 53.56; FT-IR $v / \mathrm{cm}^{-1}(\mathrm{KBr}) 2923,2852,1728,1663,1633,1510,1472,1386,1221$, 1118, 1030, 1001, 800, 750, 635, 527; UV-vis $\left(\mathrm{CHCl}_{3}\right) \lambda_{\max } / \mathrm{nm}(\log \varepsilon) 260$ (5.12), 328 (4.14), 404 (3.01), 440 (2.65), 704 (1.95); MALDI-TOF MS $m / z$ calcd for $\mathrm{C}_{77} \mathrm{H}_{14} \mathrm{~N}_{2} \mathrm{O}_{3}$ [M] $]^{-} 1014$. 1010, found 1014.1019.

\section{Synthesis and spectral data of compound 3d}

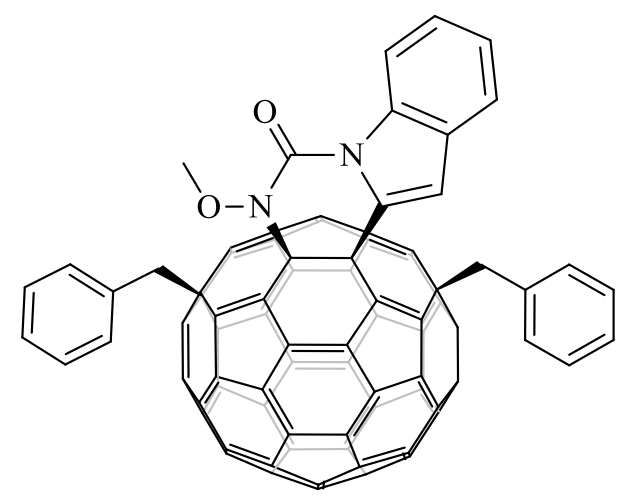

3d

$20.0 \mathrm{mg}(0.022 \mathrm{mmol})$ of $\mathbf{2 a}$ was electroreduced by CPE at $-1.14 \mathrm{~V}$ vs SCE in $25 \mathrm{~mL}$ of ODCB containing 0.1 M TBAP under argon atmosphere at room temperature. The electrolysis was terminated when the theoretical number of coulombs required for a full conversion of $\mathbf{2 a}$ to $\mathbf{2} \mathbf{a}^{2-}$ was reached. Then, the dianionic $\mathbf{2 a}$ was reacted with benzyl bromide $(26.2 \mu \mathrm{L}, 0.22 \mathrm{mmol})$ and $\mathrm{NaH}(57-63 \%$ oil dispersion, $17.6 \mathrm{mg}, 0.44 \mathrm{mmol})$ at $40{ }^{\circ} \mathrm{C}$ for $12 \mathrm{~h}$. The reaction mixture was filtered through a silica gel (200-300 mesh) plug with $\mathrm{CS}_{2} / \mathrm{CH}_{2} \mathrm{Cl}_{2}(1: 1, \mathrm{v} / \mathrm{v})$ to remove the electrolyte. After evaporation in vacuo, the residue was separated by silica gel (400-500 mesh) column with $\mathrm{CS}_{2} / \mathrm{CH}_{2} \mathrm{Cl}_{2}(4: 1, \mathrm{v} / \mathrm{v})$ to afford 3d $(12.7 \mathrm{mg}, 53 \%)$ as an amorphous brown solid. ${ }^{1} \mathrm{H}$ NMR (400 MHz, $\mathrm{CS}_{2}$ with DMSO- $d_{6}$ as the external deuterium lock and containing TMS as the reference) $\delta 8.35(\mathrm{~d}, J=8.2 \mathrm{~Hz}, 1 \mathrm{H}), 7.61(\mathrm{~d}, J=7.7 \mathrm{~Hz}, 1 \mathrm{H}), 7.43-7.37(\mathrm{~m}, 1 \mathrm{H}), 7.34-7.29(\mathrm{~m}$, 3H), 7.28-7.19 (m, 8H), 7.16 (d, J=1.3 Hz, 1H), 4.16-4.00 (m, 5H), 3.96-3.91 (m, 2H); ${ }^{13} \mathrm{C} \mathrm{NMR}$ $\left(101 \mathrm{MHz}, \mathrm{CS}_{2}\right.$ with DMSO- $d_{6}$ as the external deuterium lock and reference) $\delta 156.12,154.56$, $153.27,152.12,151.23,150.81,150.68,149.60$, 148.50, 148.43, 147.45, 147.32, 147.27, 147.11, $146.62,146.36,146.26,146.21,146.14,146.09$, 145.97, 145.73, 145.56, 145.39, 145.09, 144.92, 
$144.56,144.20,144.09,143.79,143.45,143.41,143.14,143.10,143.06,142.96,142.63,142.48$, $142.43,142.38,142.19,142.03,142.00,141.82$, 141.74, 141.22, 141.17, 141.13, 141.00, 139.63, 139.35, 138.78, 137.02, 136.80, 136.30, 135.78, 134.18, 133.84, 133.56, 130.65, 129.74, 129.45, $128.54,127.34,127.23,126.42,126.27,124.80,123.32,120.01,115.38,106.81,70.58,62.45$, 57.37, 57.05, 53.18, 45.73, 45.50; FT-IR $v / \mathrm{cm}^{-1}$ (KBr) 2919, 2849, 1736, 1656, 1633, 1471, 1453, 1341, 1123, 1038, 1015, 835, 750, 694, 526; UV-vis $\left(\mathrm{CHCl}_{3}\right) \lambda_{\max } / \mathrm{nm}(\log \varepsilon) 239$ (4.93), 271 (4.81), 332 (4.51), 410 (4.00), 439 (3.93); MALDI-TOF MS m/z calcd for $\mathrm{C}_{83} \mathrm{H}_{19} \mathrm{~N}_{2} \mathrm{O}$ [M-OMe] 1059.1503, found 1059.1516.

\section{Scaled-up synthesis of 2 a}

A mixture of $\mathrm{C}_{60}(360.0 \mathrm{mg}, 0.5 \mathrm{mmol}), \mathbf{1 a}(285.1 \mathrm{mg}, 1.5 \mathrm{mmol}), \mathrm{Pd}(\mathrm{OAc})_{2}(11.3 \mathrm{mg}, 0.05 \mathrm{mmol})$, $\mathrm{K}_{2} \mathrm{~S}_{2} \mathrm{O}_{8}(270.1 \mathrm{mg}, 1.0 \mathrm{mmol})$, and TFA $(0.8 \mathrm{~mL})$ was dissolved in ODCB $(20 \mathrm{~mL})$. Then the solution was stirred at $100{ }^{\circ} \mathrm{C}$ for $36 \mathrm{~h}$. The resulting solution was evaporated in vacuo and then separated on a silica gel column with $\mathrm{CS}_{2} / \mathrm{CH}_{2} \mathrm{Cl}_{2}(4: 1 \mathrm{v} / \mathrm{v})$ as the eluent to give recovered $\mathrm{C}_{60}$ (222.3 $\mathrm{mg}, 62 \%)$ and $\mathbf{2 a}(113.0 \mathrm{mg}, 25 \%)$.

\section{Mechanistic study}

\section{$5.1 \mathrm{D} / \mathrm{H}$ exchange experiment of $1 \mathrm{a}$}

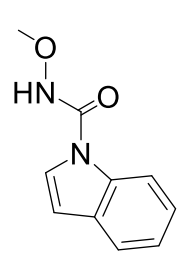

$1 \mathrm{a}$

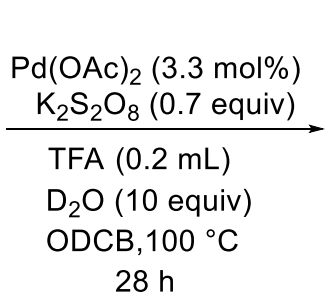

$28 \mathrm{~h}$

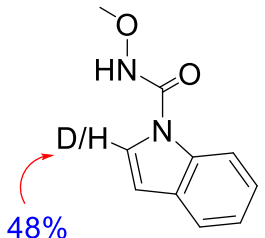

[D]-1a

Substrate 1a $(28.6 \mathrm{mg}, 0.15 \mathrm{mmol})$ was treated with $\mathrm{Pd}(\mathrm{OAc})_{2}(1.1 \mathrm{mg}, 0.005 \mathrm{mmol}), \mathrm{K}_{2} \mathrm{~S}_{2} \mathrm{O}_{8}$ (27.0 mg, $0.10 \mathrm{mmol})$, TFA $(0.2 \mathrm{~mL})$, and $\mathrm{D}_{2} \mathrm{O}(27 \mu \mathrm{L}, 1.5 \mathrm{mmol})$ in ODCB $(5 \mathrm{~mL})$ at $100{ }^{\circ} \mathrm{C}$ for $28 \mathrm{~h}$. The solvent was removed in vacuo, and the residue was diluted with $20 \mathrm{~mL}$ of EtOAc, filtered through a celite pad, washed with saturated $\mathrm{NH}_{4} \mathrm{Cl}$ solution, and dried over anhydrous $\mathrm{Na}_{2} \mathrm{SO}_{4}$. The solution was concentrated, and the resulting residue was purified by column chromatography on silica gel to provide the deuterated product. The deuterium incorporation at the $\mathrm{C} 2$ atom was $48 \%$ based on the ${ }^{1} \mathrm{H}$ NMR integrals. This $\mathrm{D} / \mathrm{H}$ exchange experiment of $1 \mathrm{a}$ indicated that the carboxamide-directed $\mathrm{C}-\mathrm{H}$ activation indeed occurred to give the palladacycle intermediate, which underwent deuterium incorporation at the $\mathrm{C} 2$ atom in the presence of $\mathrm{D}_{2} \mathrm{O}$. 


\subsection{Kinetic isotope effect (KIE) study}

2-Deuterium indole was prepared according to the procedure reported in the literatue. ${ }^{1}[\mathbf{D}]-\mathbf{1 a}$ was synthesized with $100 \%$ D-incorporation from 2-deuterium indole by following the reported procedure. $^{2}$ The NMR spectral data matched the published data. ${ }^{3}$

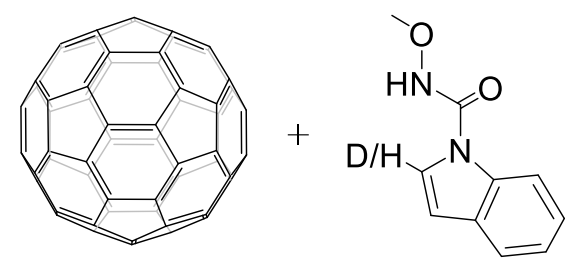

1a or [D]-1a

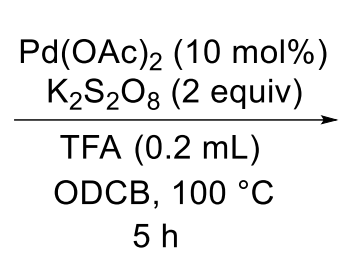

$\mathrm{KIE}=1.23$

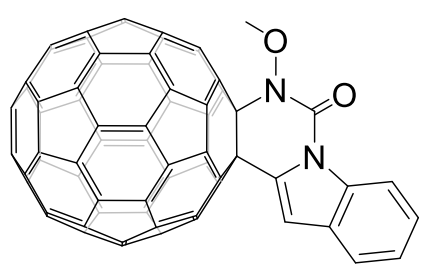

2a

Two sets of reactions were carried out in a parallel manner. By following the general procedure for the synthesis of $\mathbf{2}$, the reaction of $\mathrm{C}_{60}(36.0 \mathrm{mg}, 0.05 \mathrm{mmol})$ with $\mathbf{1 a}(28.5 \mathrm{mg}, 0.15 \mathrm{mmol})$, $\mathrm{Pd}(\mathrm{OAc})_{2}(1.2 \mathrm{mg}, 0.005 \mathrm{mmol}), \mathrm{K}_{2} \mathrm{~S}_{2} \mathrm{O}_{8}(27.1 \mathrm{mg}, 0.10 \mathrm{mmol})$, and TFA $(0.2 \mathrm{~mL})$ in ODCB (5 $\mathrm{mL})$ at $100{ }^{\circ} \mathrm{C}$ for $5 \mathrm{~h}$ afforded $\mathbf{2 a}(4.3 \mathrm{mg}, 9 \%)$.

By following the general procedure, the reaction of $\mathrm{C}_{60}(36.0 \mathrm{mg}, 0.05 \mathrm{mmol})$ with [D]-1a (28.5 $\mathrm{mg}, 0.15 \mathrm{mmol}), \mathrm{Pd}(\mathrm{OAc})_{2}(1.2 \mathrm{mg}, 0.005 \mathrm{mmol}), \mathrm{K}_{2} \mathrm{~S}_{2} \mathrm{O}_{8}(27.2 \mathrm{mg}, 0.10 \mathrm{mmol})$, and TFA $(0.2$ $\mathrm{mL})$ in ODCB $(5 \mathrm{~mL})$ at $100{ }^{\circ} \mathrm{C}$ for $5 \mathrm{~h}$ afforded $2 \mathrm{a}(3.5 \mathrm{mg}, 7 \%)$.

The KIE was 1.23 , as calculated from the ratio of the product yields for the two parallel experiments.

\section{References}

(1) Sevov, C. S.; Hartwig, J. F. J. Am. Chem. Soc. 2013, 135, 2116.

(2) Zhang, W.; Wei, J.; Fu, S.; Lin, D.; Jiang, H.; Zeng, W. Org. Lett. 2015, 17, 1349.

(3) Zheng, J.; Zhang, Y.; Cui, S. Org. Lett. 2014, 16, 3560. 
7. NMR spectra of compounds $2 \mathrm{a}-\mathrm{n}, 3 \mathrm{3}-\mathrm{d}$, and $[\mathrm{D}]-\mathbf{- 1 a}$

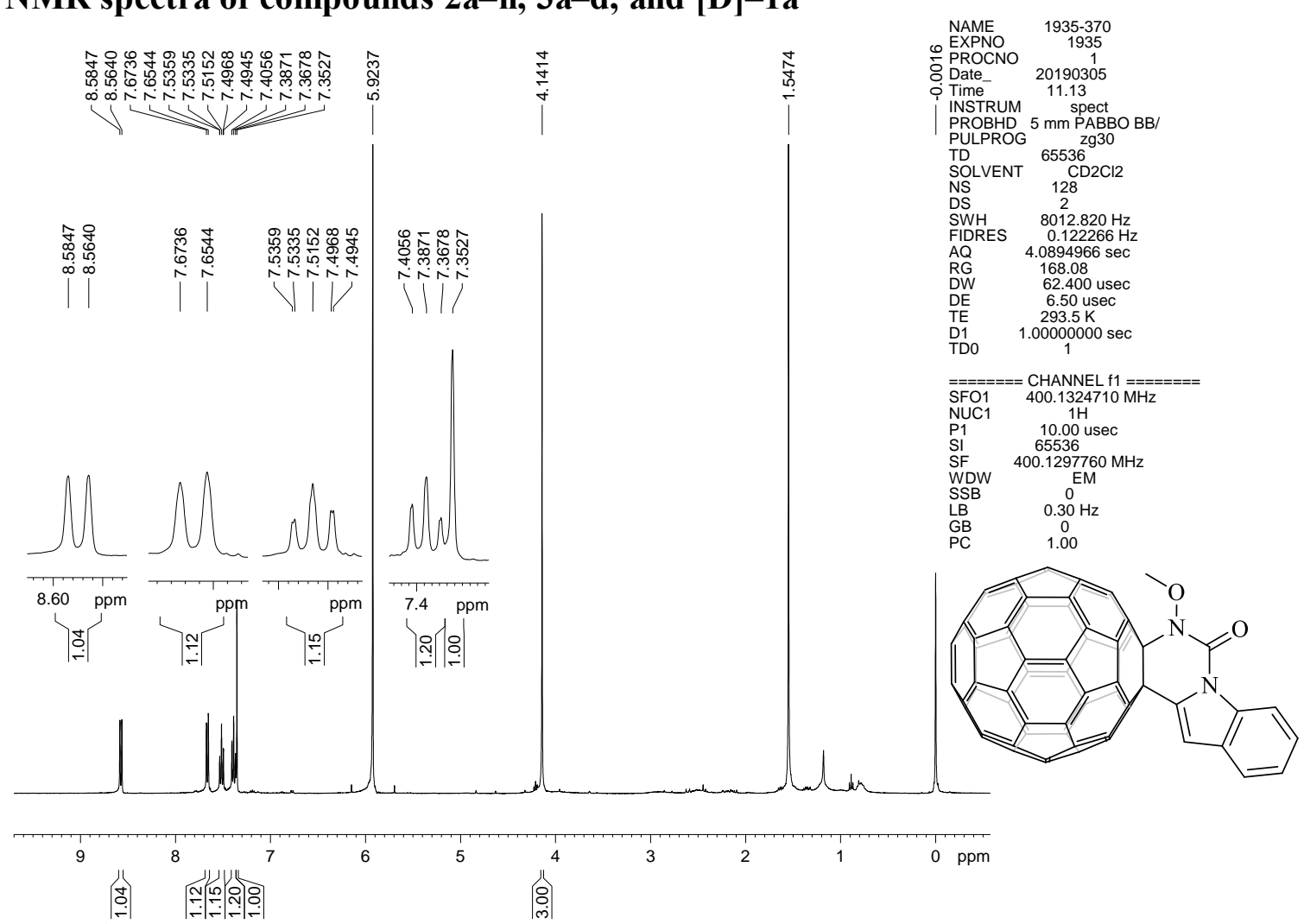

Figure S1 ${ }^{1} \mathrm{H}$ NMR (400 MHz, $\mathrm{CS}_{2} / \mathrm{CDCl}_{2} \mathrm{CDCl}_{2} 2: 3$ ) of Compound 2a

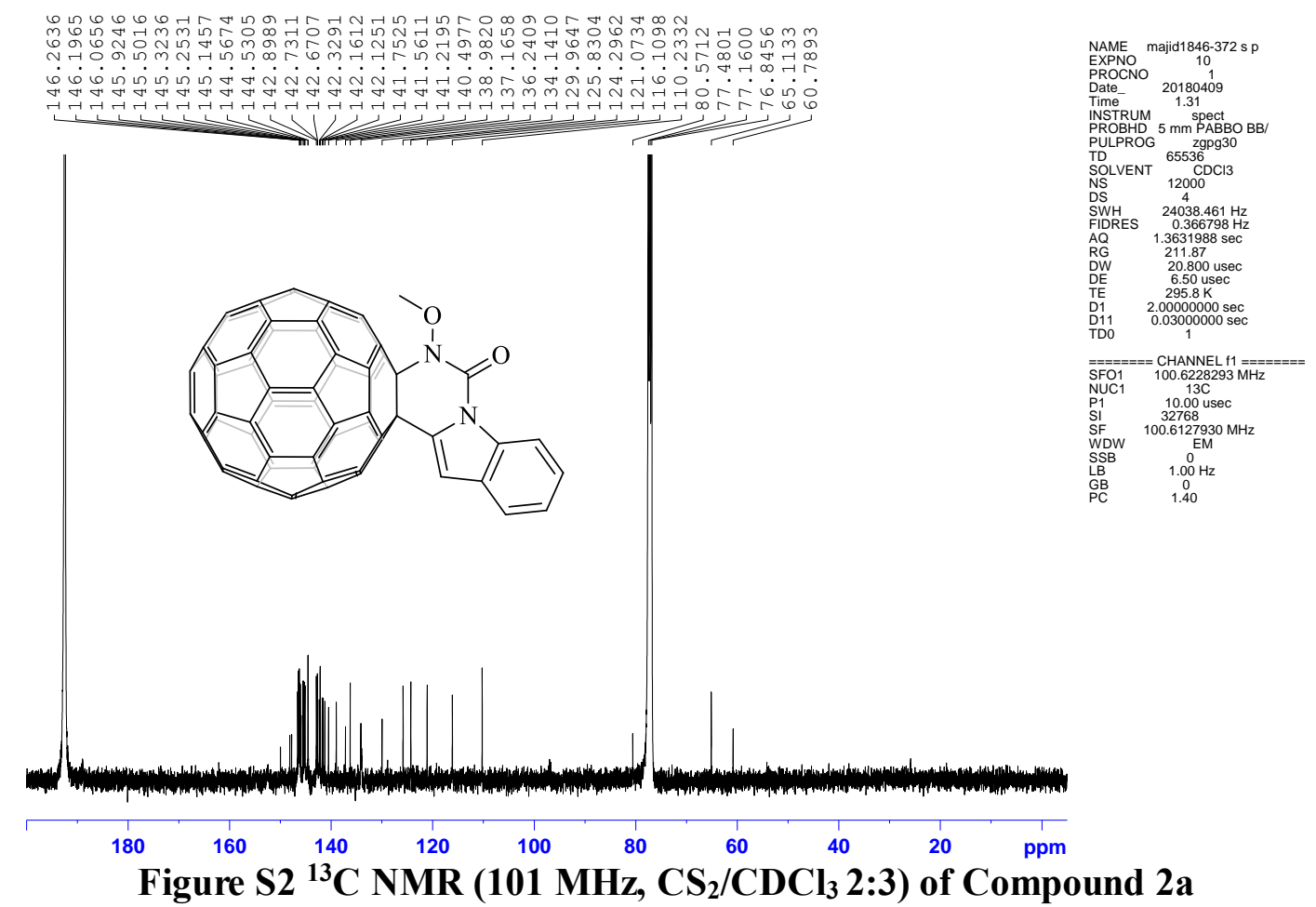



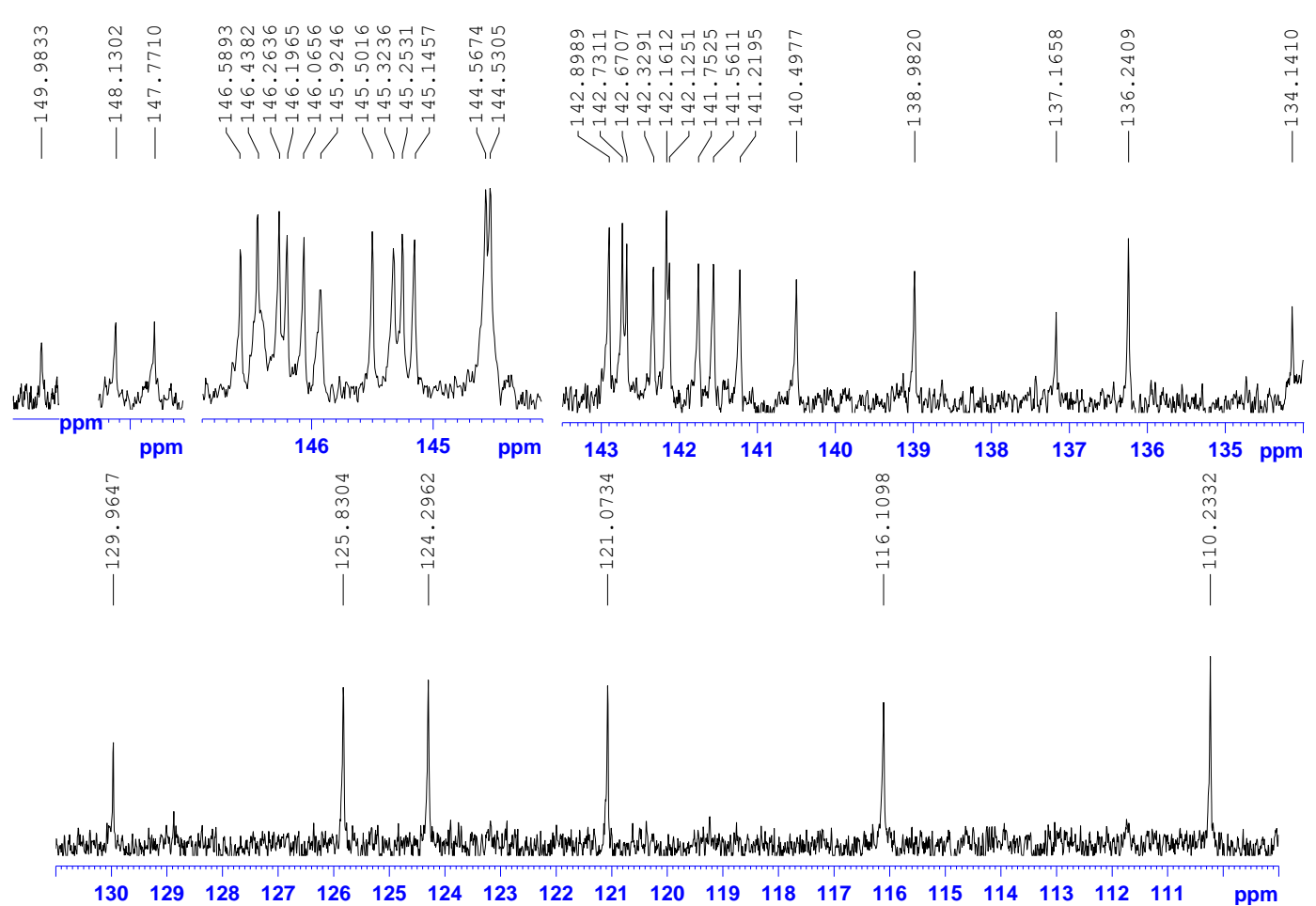

Figure S3 Expanded $\left.{ }^{13} \mathrm{C} \mathrm{NMR} \mathrm{(101} \mathrm{MHz}, \mathrm{CS}_{2} / \mathrm{CDCl}_{3} 2: 3\right)$ of Compound 2a

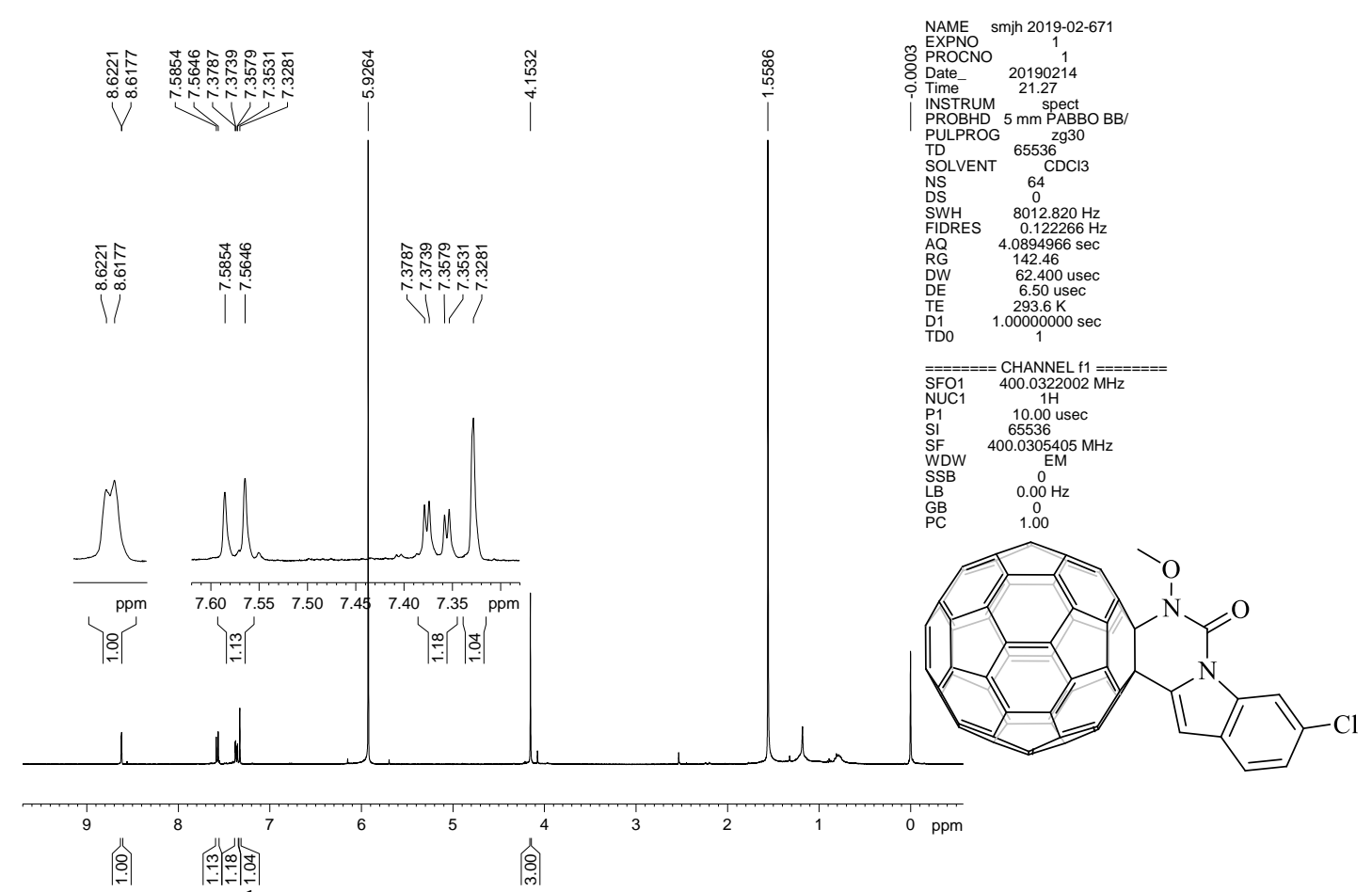

Figure S4 ${ }^{1} \mathrm{H}$ NMR (400 MHz, $\mathrm{CS}_{2} / \mathrm{CDCl}_{2} \mathrm{CDCl}_{2} 2: 3$ ) of Compound $2 \mathrm{~b}$ 


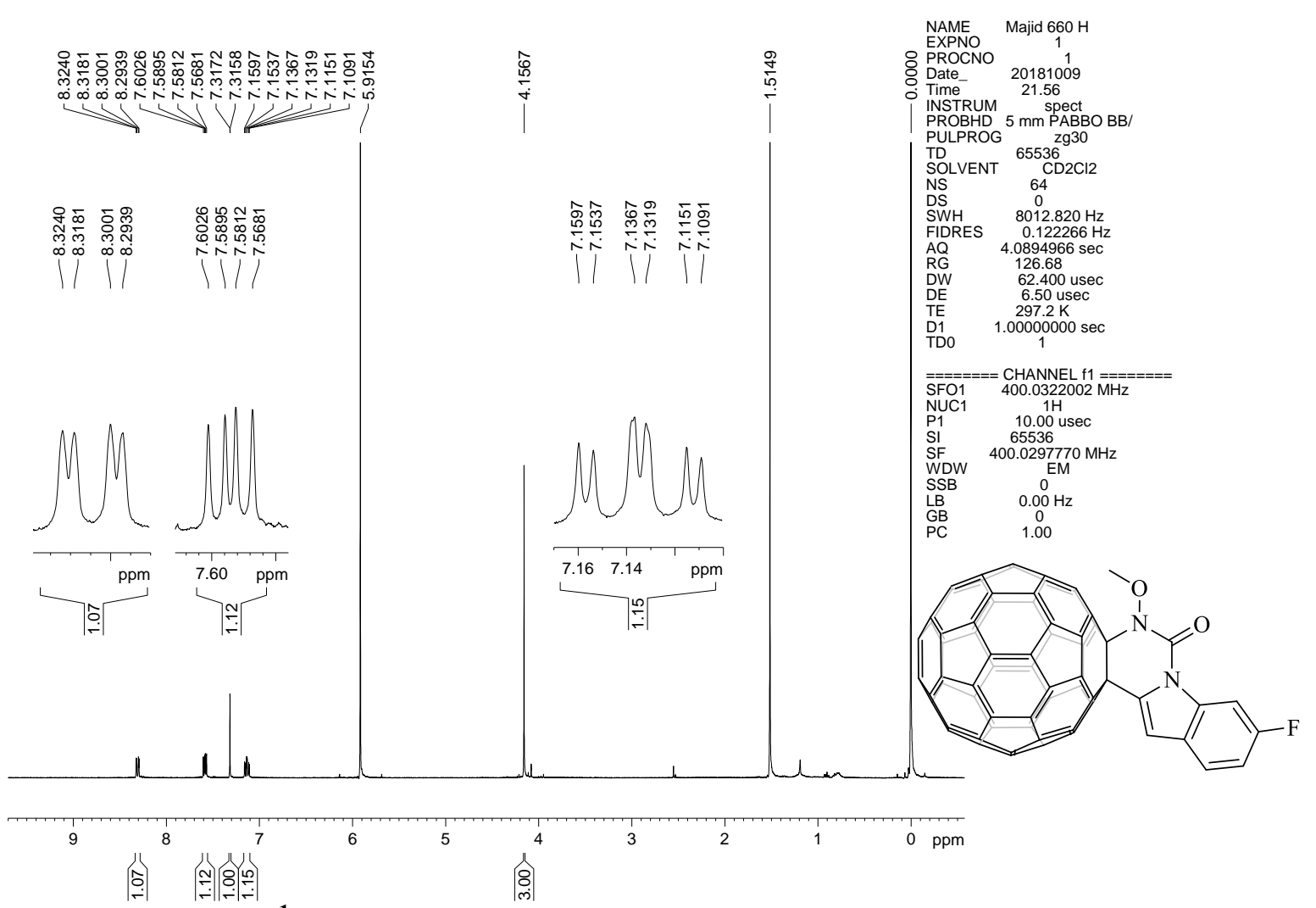

Figure S5 ${ }^{1} \mathrm{H}$ NMR (400 MHz, $\mathrm{CS}_{2} / \mathrm{CDCl}_{2} \mathrm{CDCl}_{2} 2: 3$ ) of Compound 2c

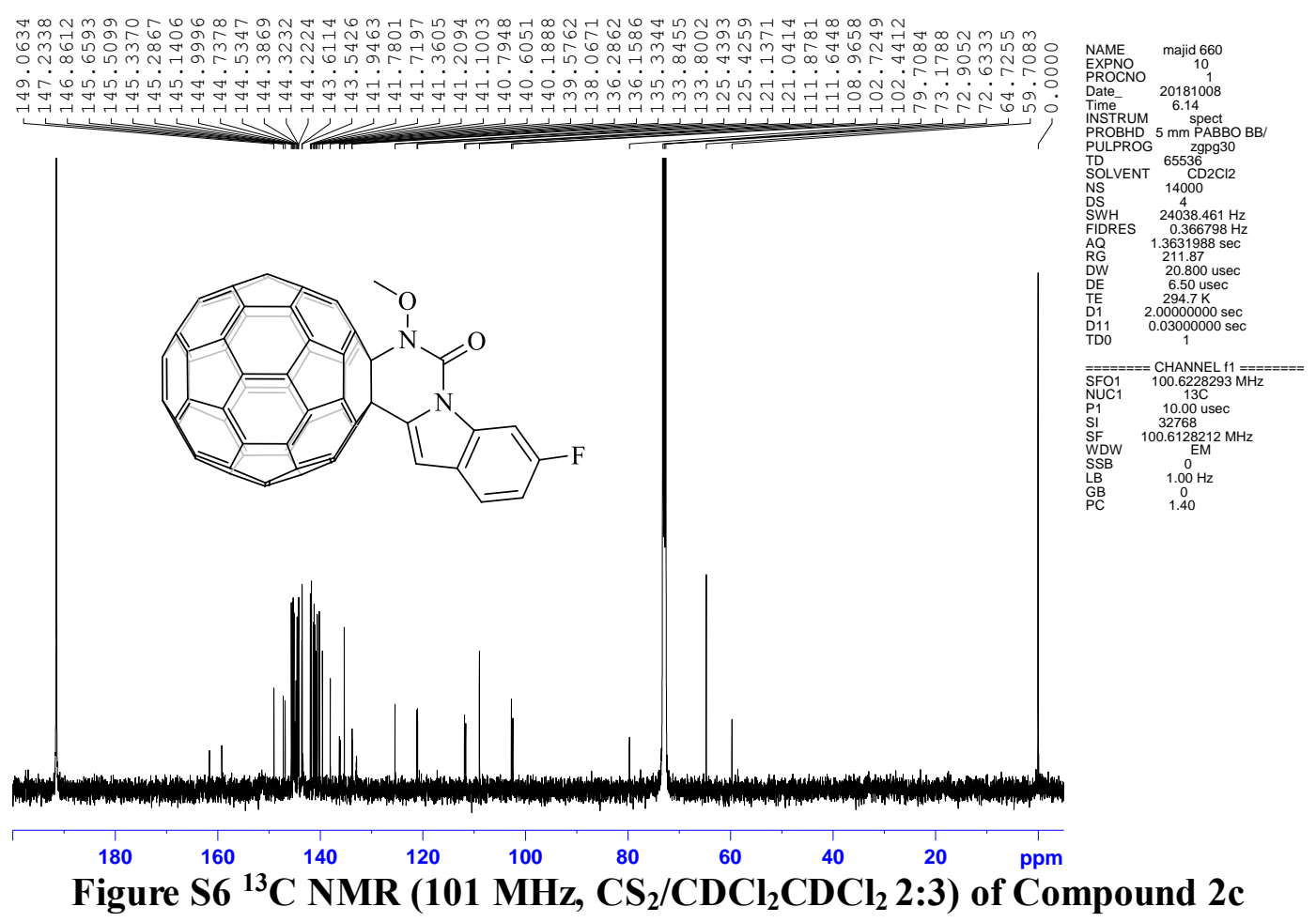



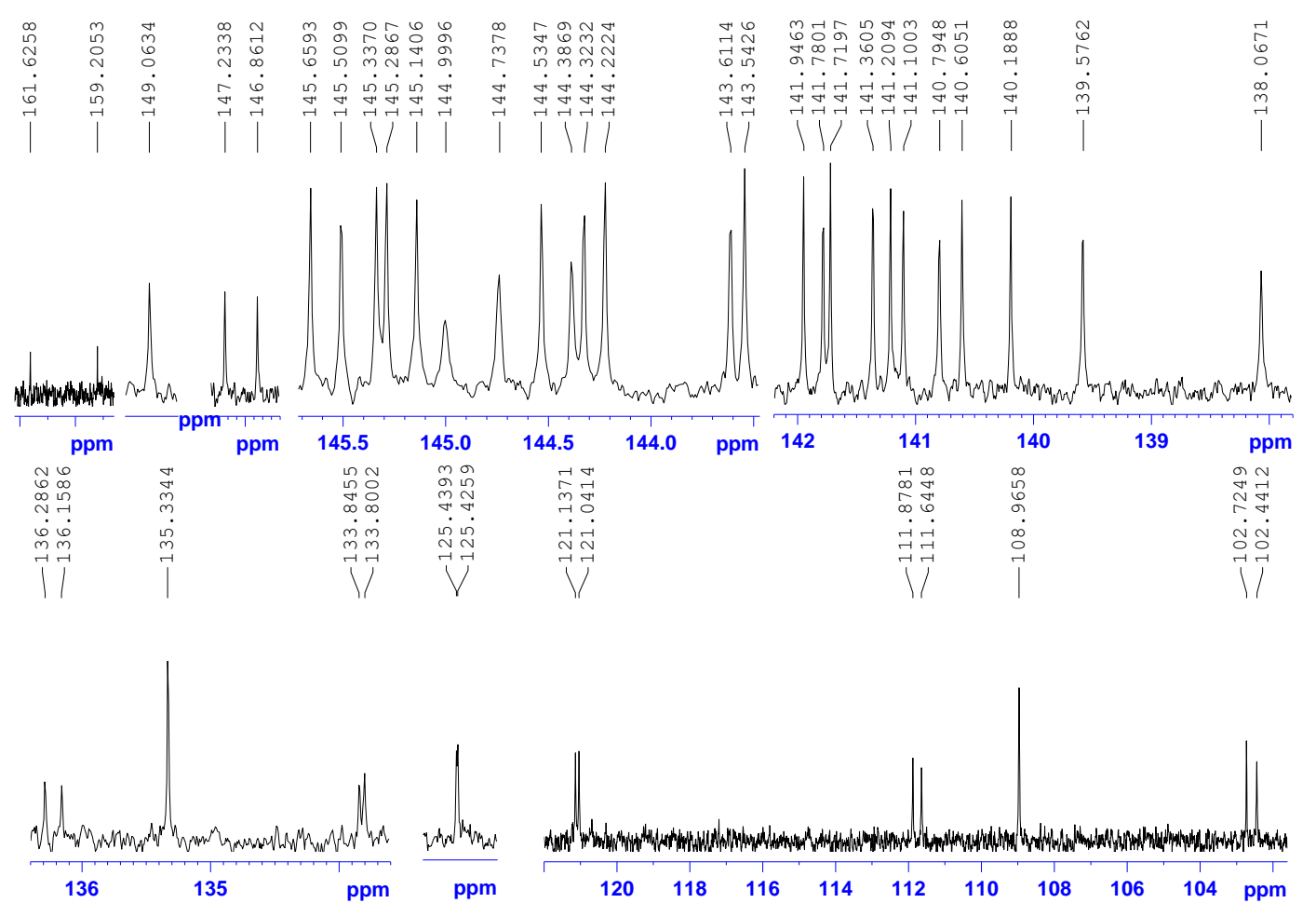

Figure S7 Expanded ${ }^{13} \mathrm{C}$ NMR (101 MHz, $\left.\mathrm{CS}_{2} / \mathrm{CDCl}_{2} \mathrm{CDCl}_{2} 2: 3\right)$ of $\mathrm{Compound} 2 \mathrm{c}$

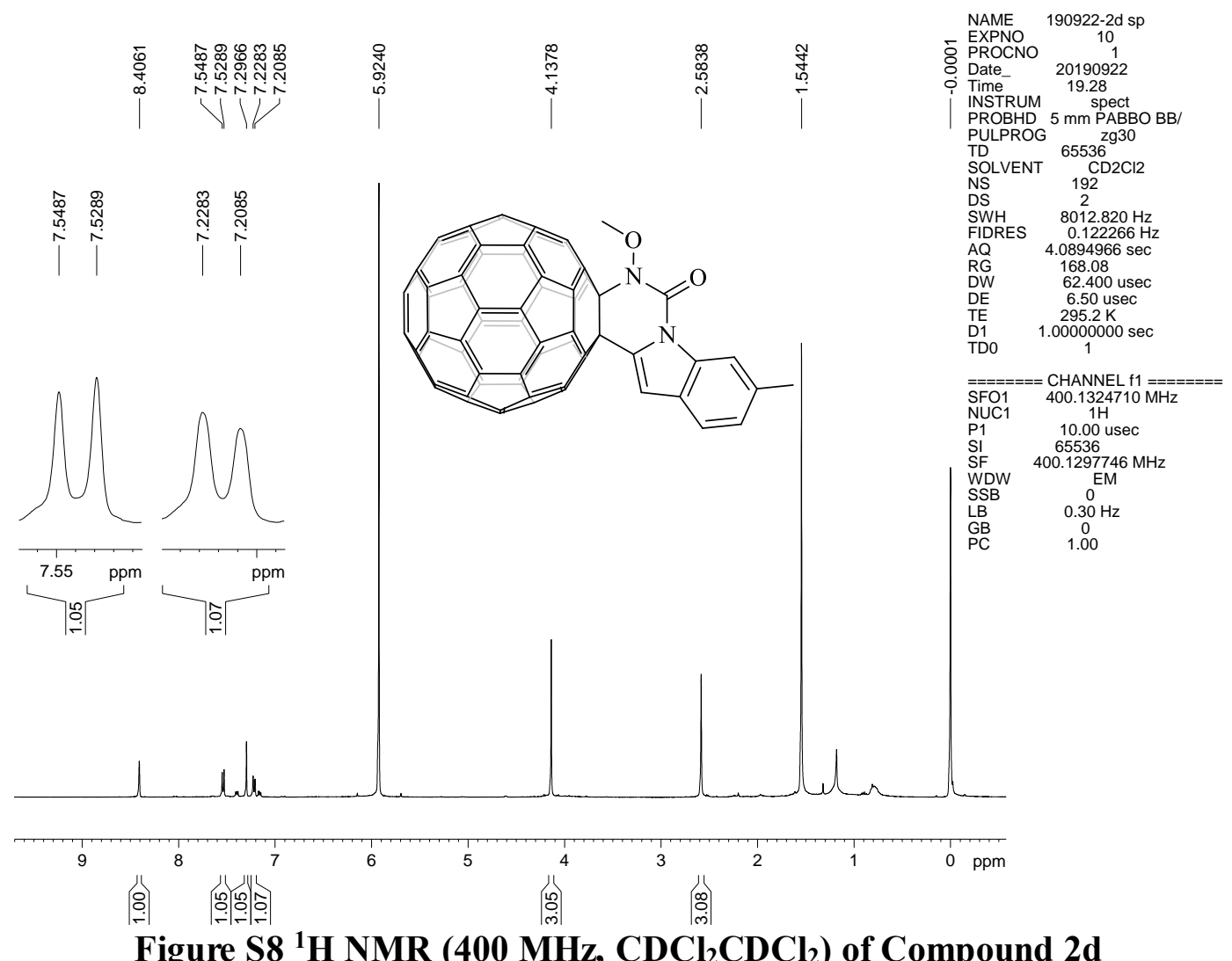



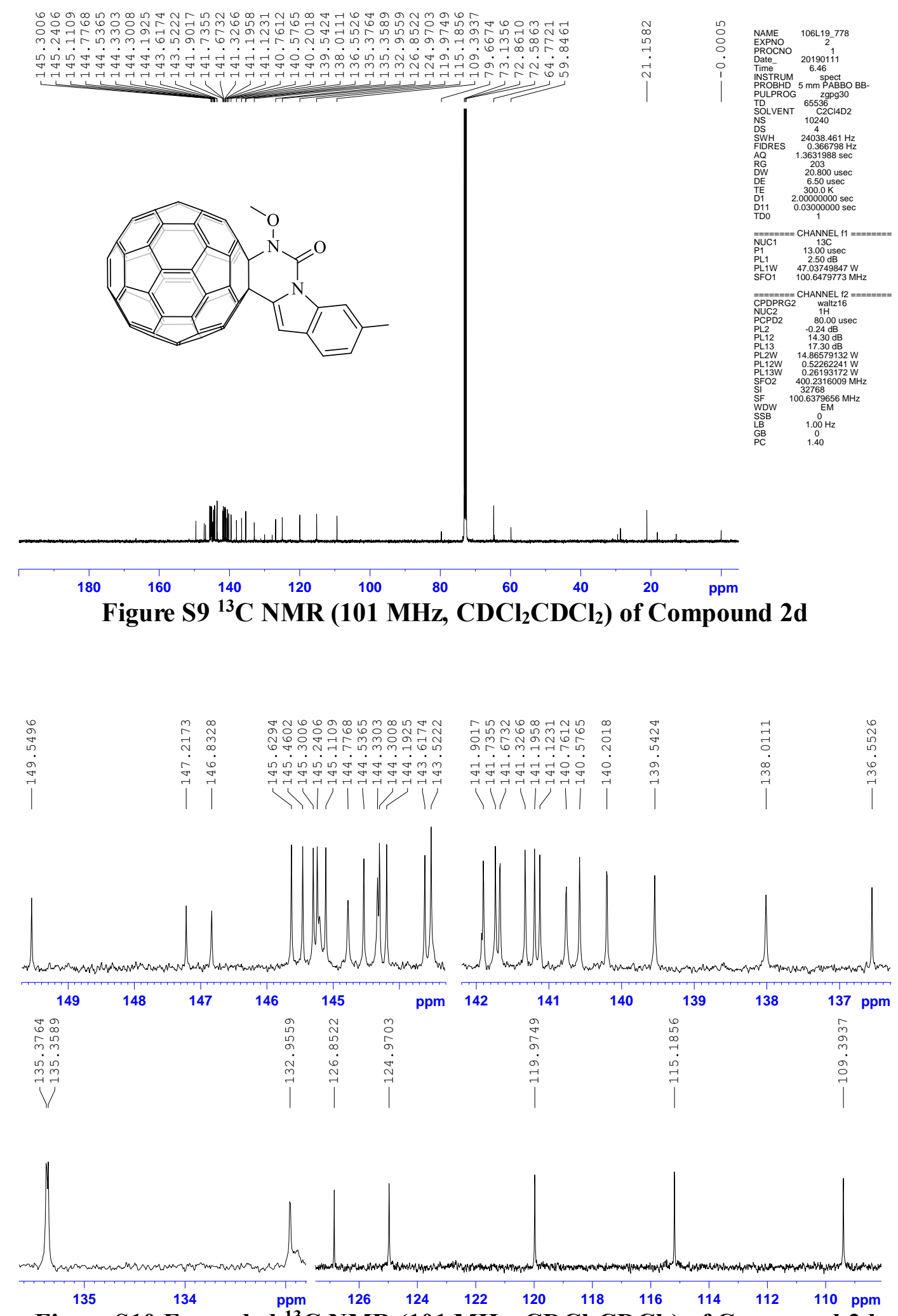

Figure S10 Expanded ${ }^{13} \mathrm{C}$ NMR (101 $\left.\mathrm{MHz}, \mathrm{CDCl}_{2} \mathrm{CDCl}_{2}\right)$ of Compound $2 \mathrm{~d}$ 


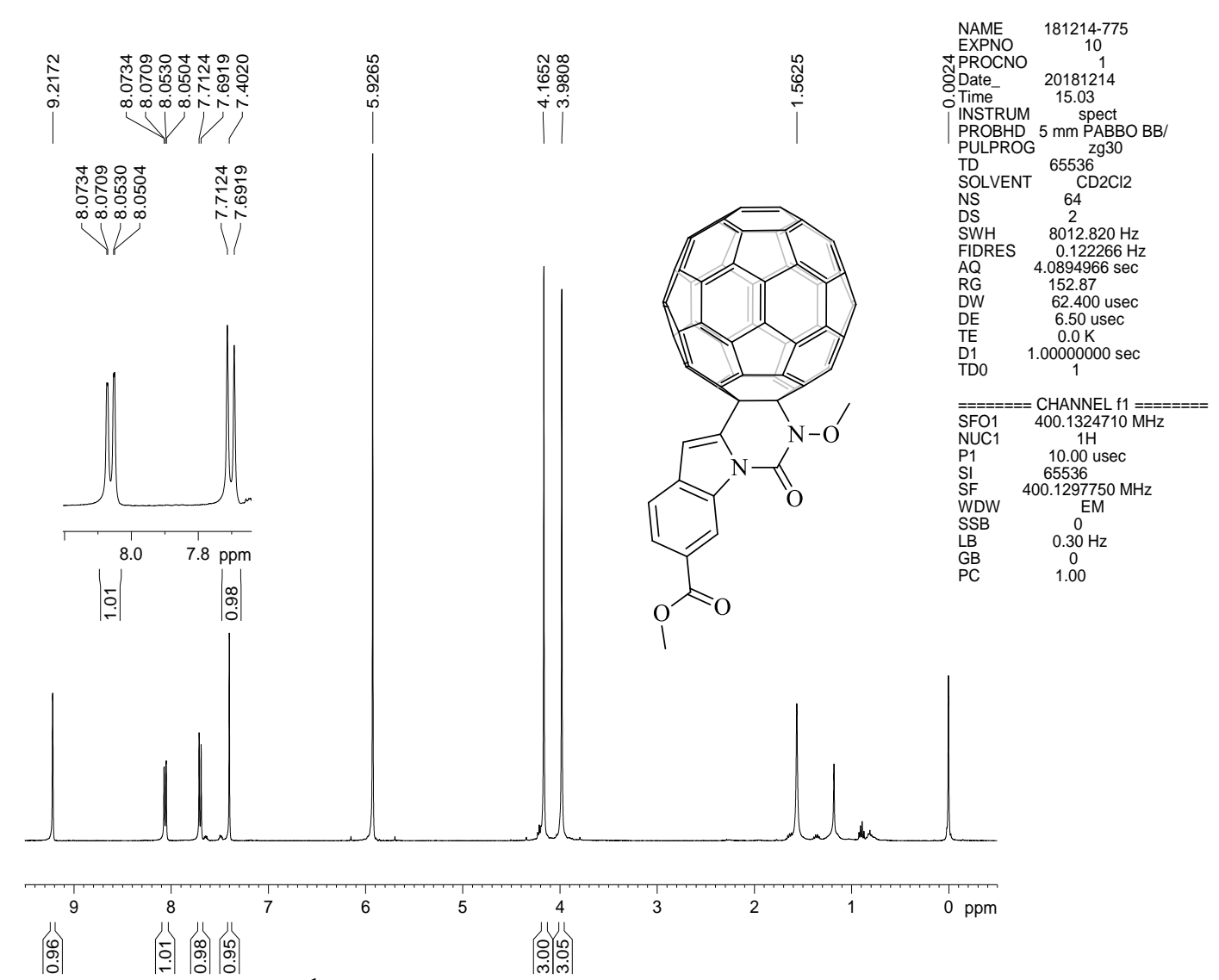

Figure S11 ${ }^{1} \mathrm{H}$ NMR (400 $\left.\mathrm{MHz}, \mathrm{CDCl}_{2} \mathrm{CDCl}_{2}\right)$ of Compound $2 \mathrm{e}$

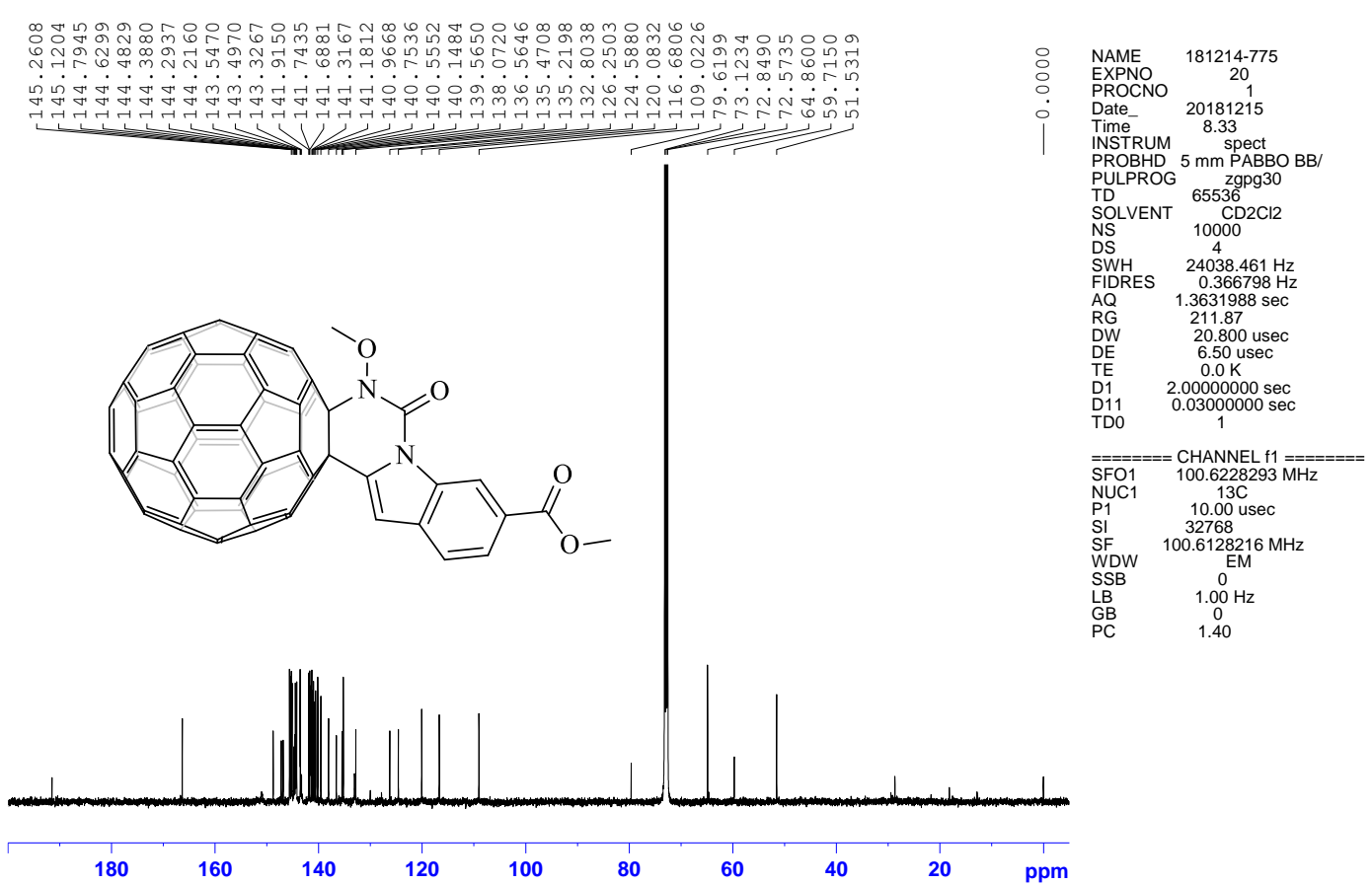

Figure $\mathrm{S12}^{13} \mathrm{C}$ NMR (101 $\left.\mathrm{MHz}, \mathrm{CDCl}_{2} \mathrm{CDCl}_{2}\right)$ of $\mathrm{Compound} 2 \mathrm{e}$ 

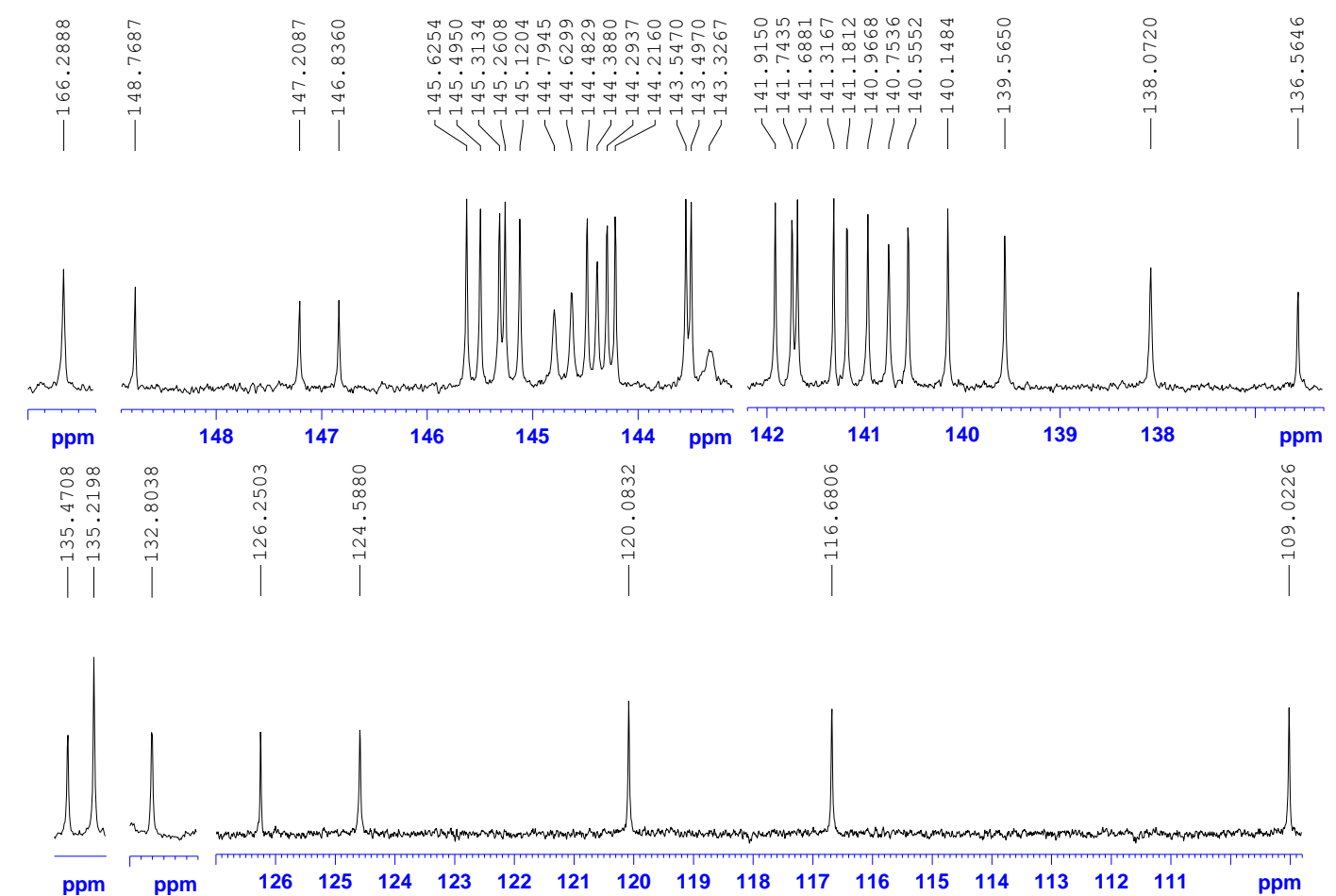
Figure S13 Expanded ${ }^{13} \mathrm{C}$ NMR (101 $\left.\mathrm{MHz}, \mathrm{CDCl}_{2} \mathrm{CDCl}_{2}\right)$ of Compound $2 \mathrm{e}$

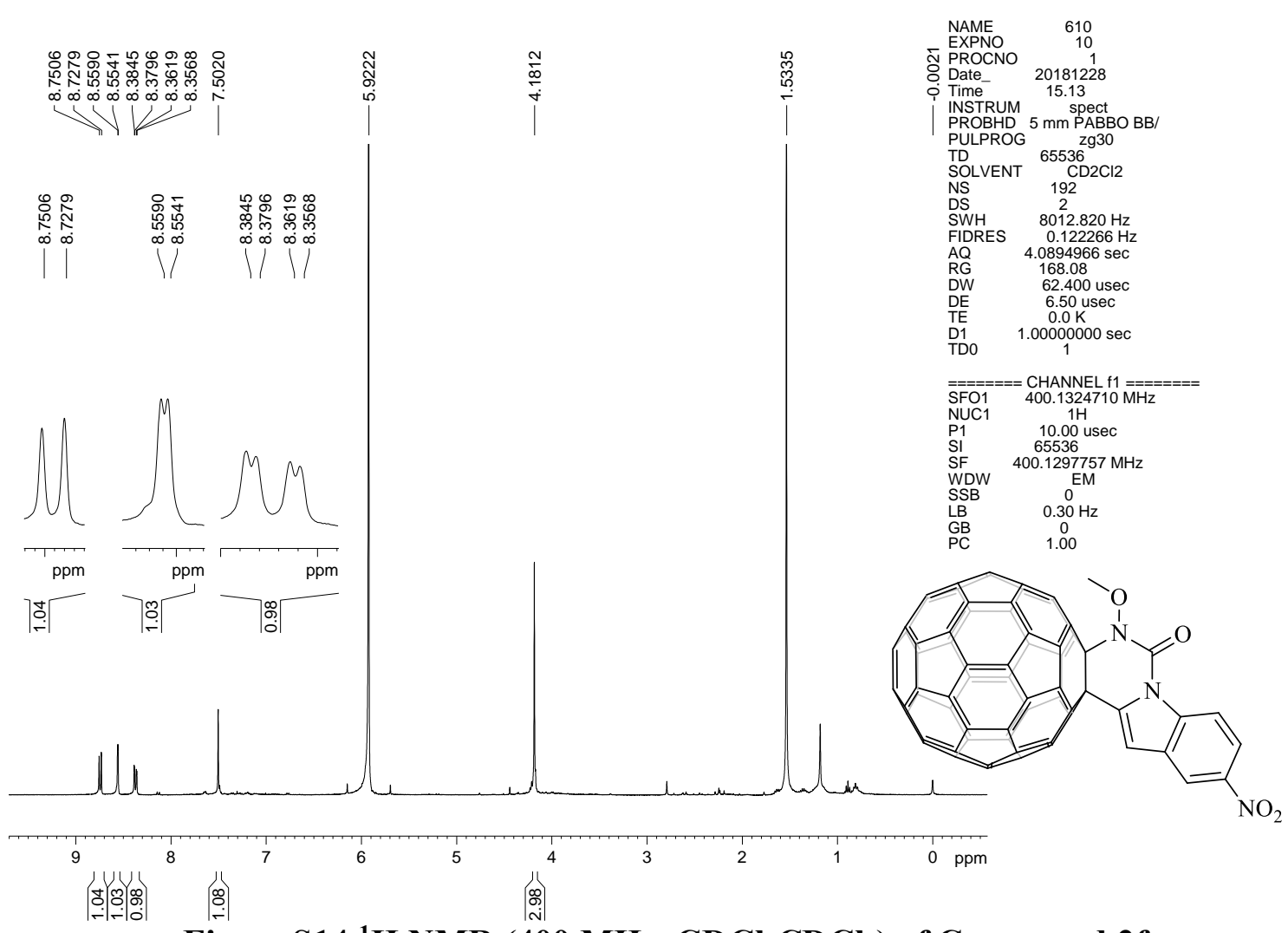

Figure S14 ${ }^{1} \mathrm{H}$ NMR (400 $\left.\mathrm{MHz}, \mathrm{CDCl}_{2} \mathrm{CDCl}_{2}\right)$ of Compound $2 \mathrm{f}$ 


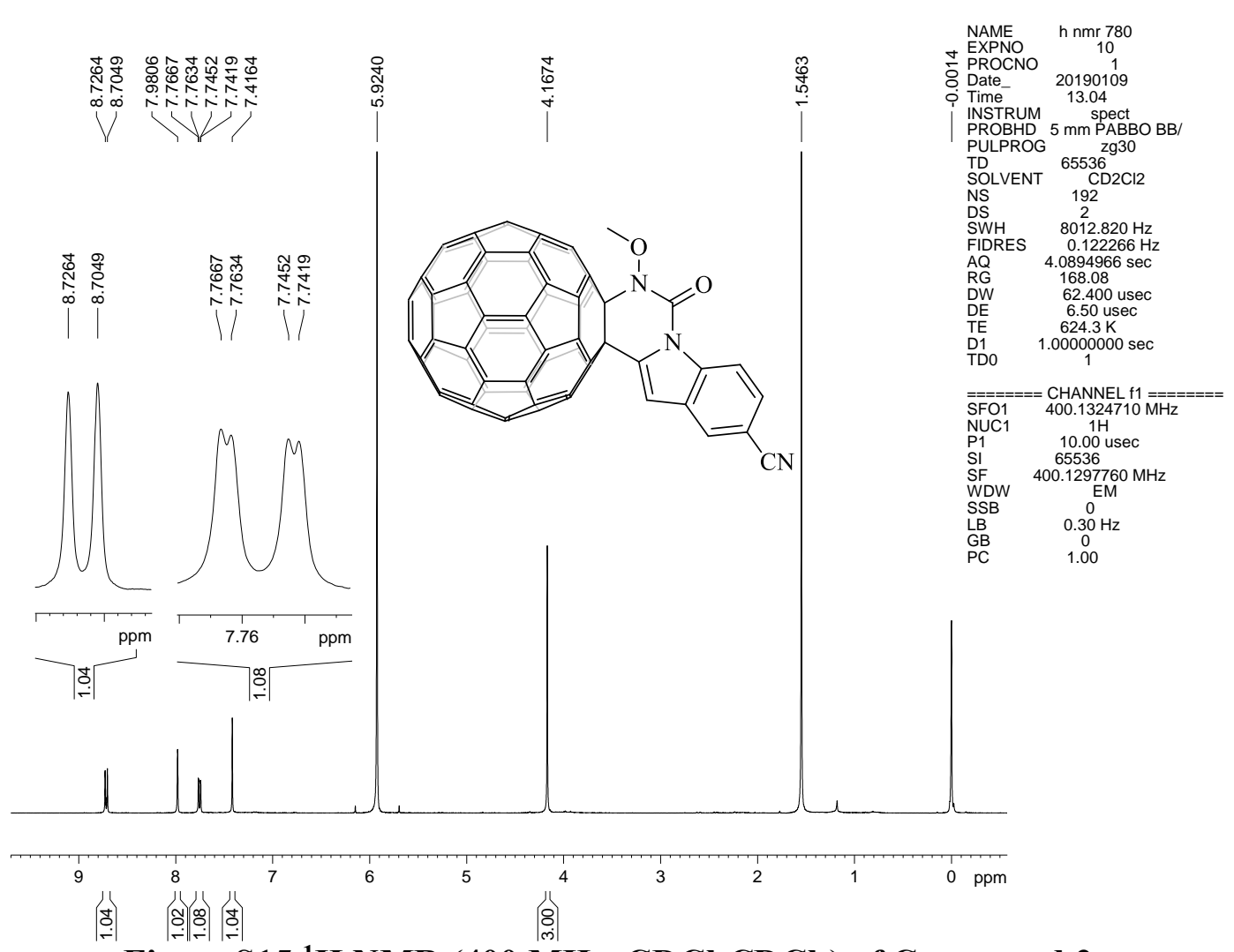

Figure $\mathrm{S}^{15}{ }^{1} \mathrm{H}$ NMR (400 MHz, $\left.\mathrm{CDCl}_{2} \mathrm{CDCl}_{2}\right)$ of $\mathrm{Compound} 2 \mathrm{~g}$

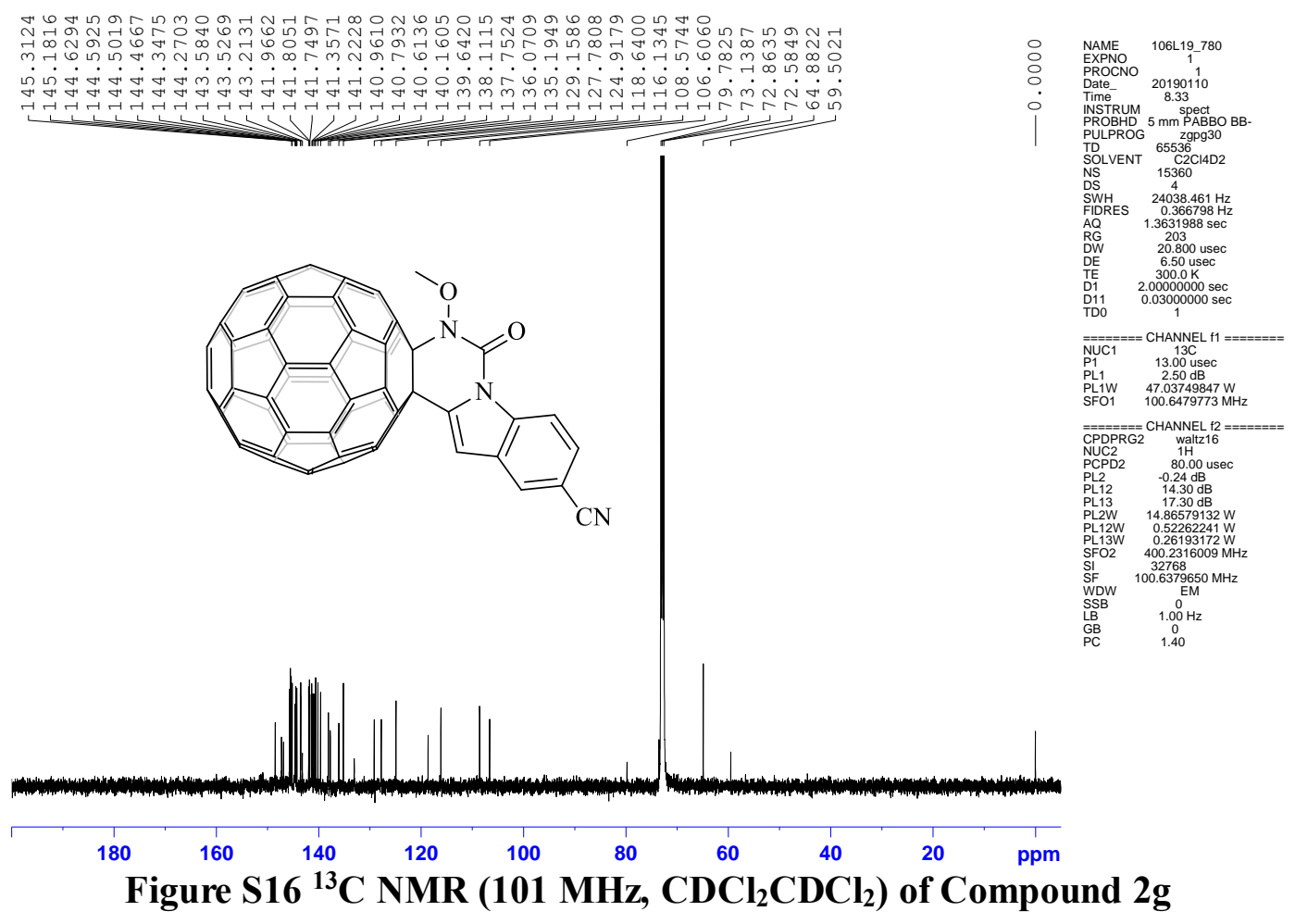



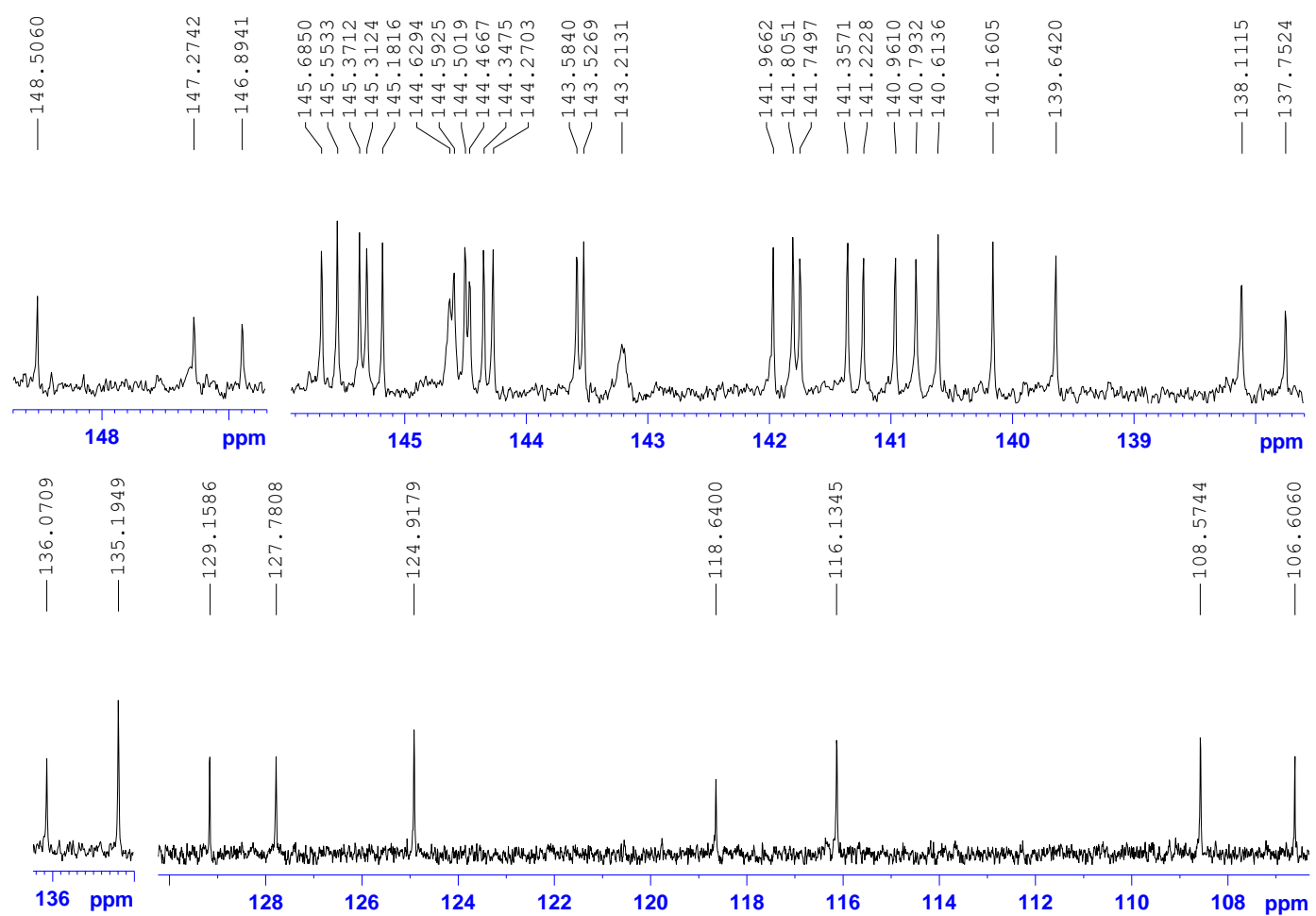
Figure S17 Expanded ${ }^{13} \mathrm{C}$ NMR (101 $\left.\mathrm{MHz}, \mathrm{CDCl}_{2} \mathrm{CDCl}_{2}\right)$ of $\mathrm{Compound} 2 \mathrm{~g}$

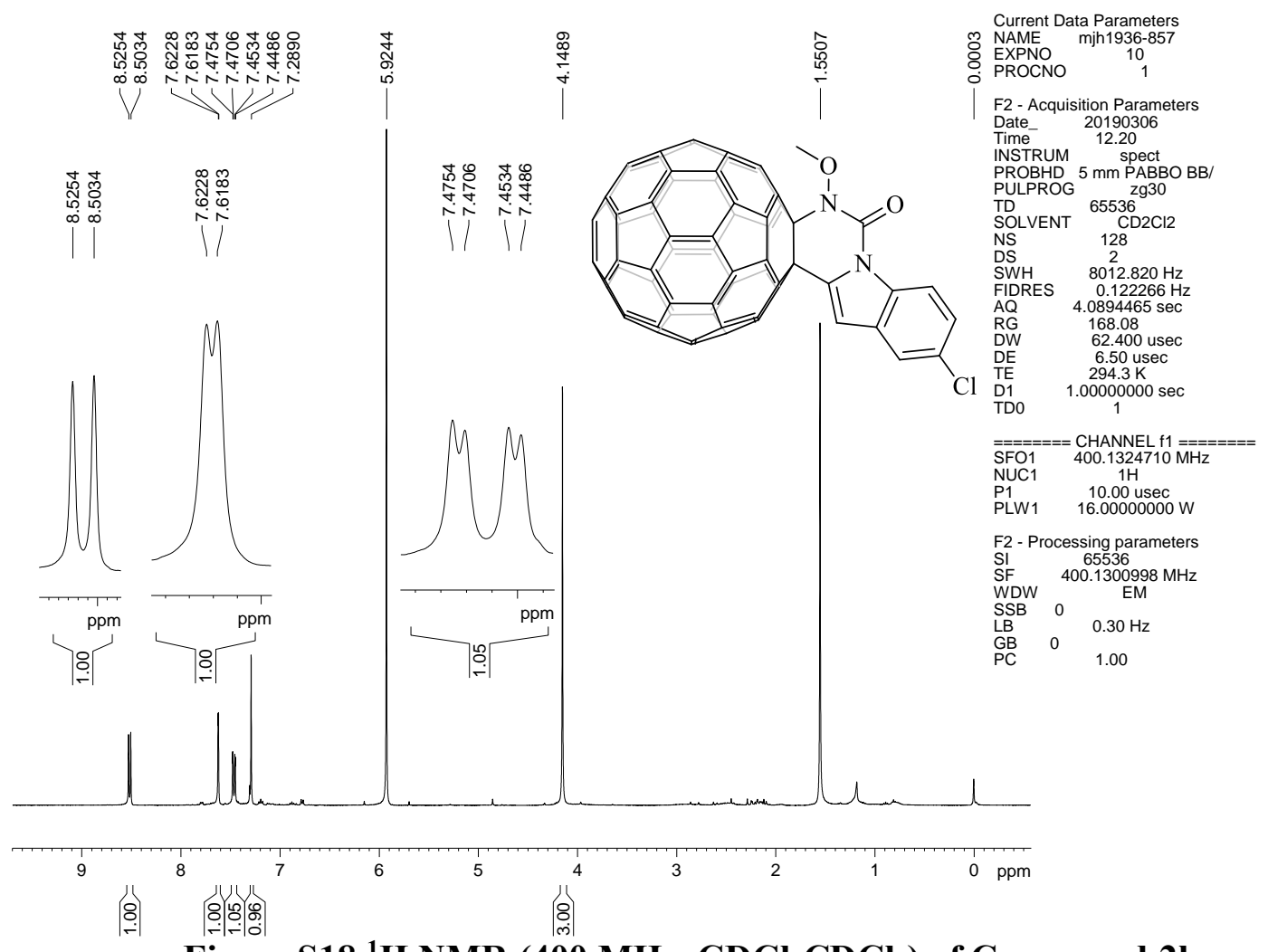

Figure S18 ${ }^{1} \mathrm{H}$ NMR (400 $\left.\mathrm{MHz}, \mathrm{CDCl}_{2} \mathrm{CDCl}_{2}\right)$ of Compound $2 \mathrm{~h}$ 

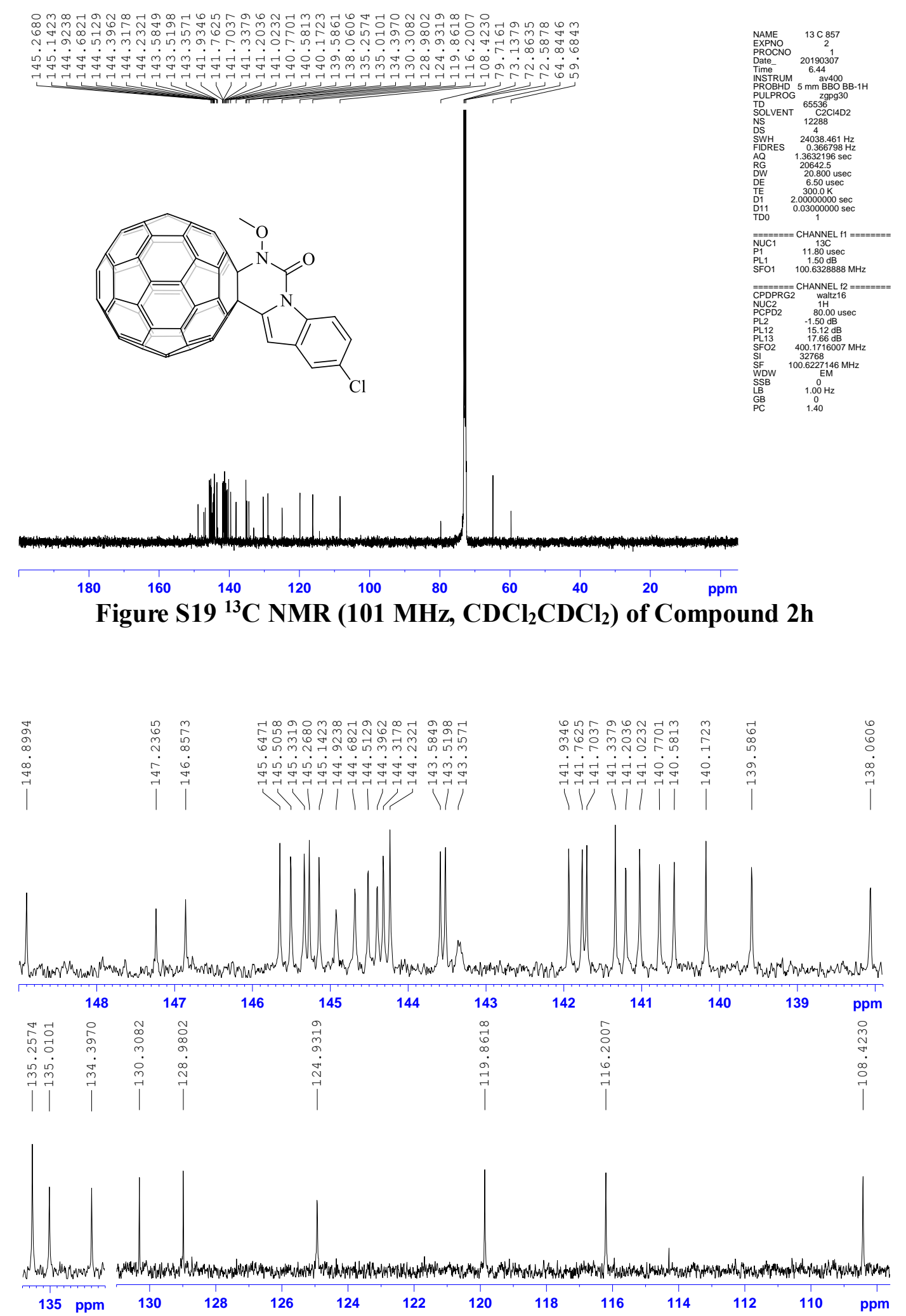

Figure S20 Expanded ${ }^{13} \mathrm{C}$ NMR (101 $\left.\mathrm{MHz}, \mathrm{CDCl}_{2} \mathrm{CDCl}_{2}\right)$ of Compound $2 \mathrm{~h}$ 

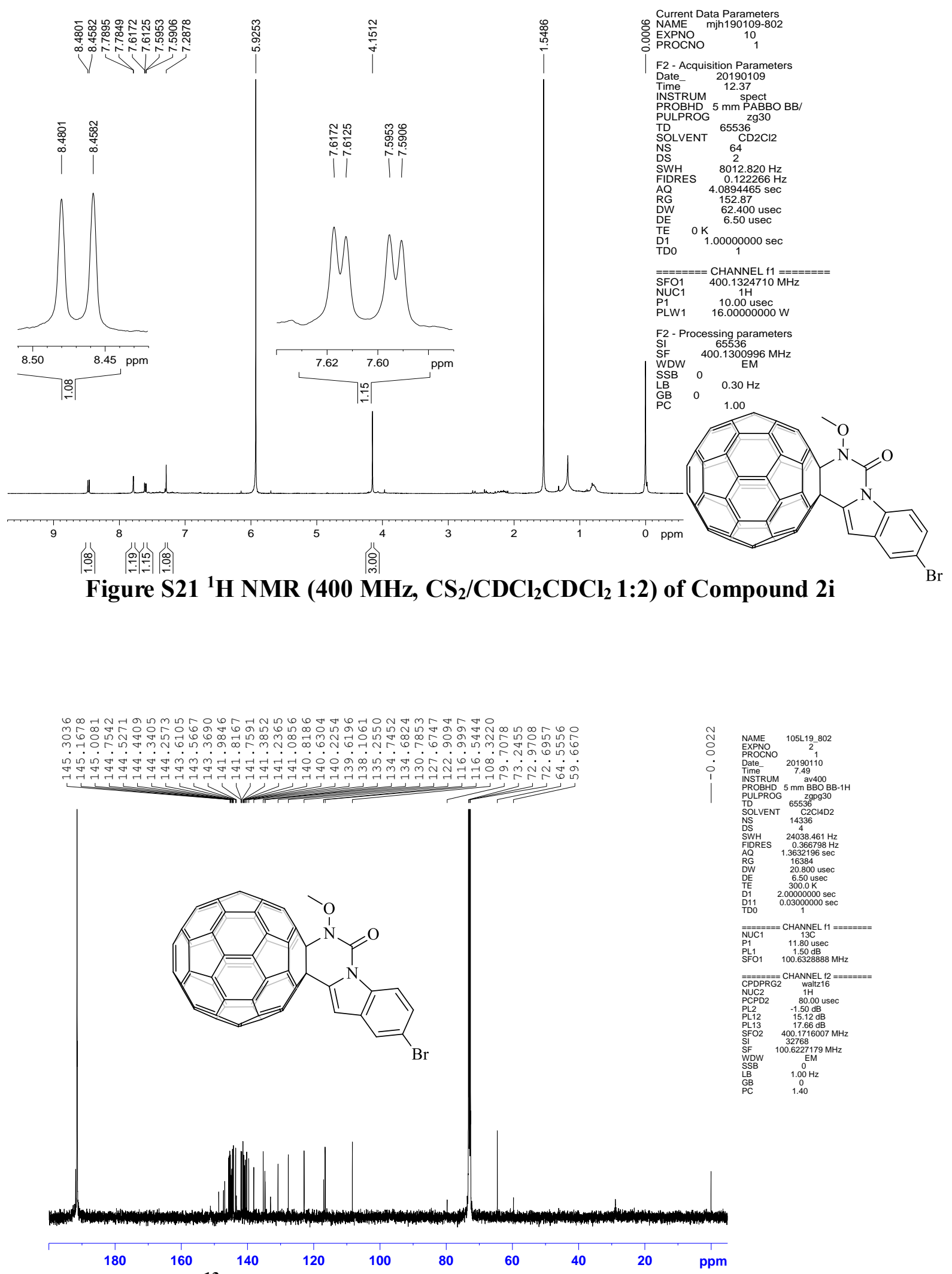

Figure S22 ${ }^{13} \mathrm{C}$ NMR (101 MHz, $\left.\mathrm{CS}_{2} / \mathrm{CDCl}_{2} \mathrm{CDCl}_{2} 1: 2\right)$ of $\mathrm{Compound} 2 \mathrm{i}$ 


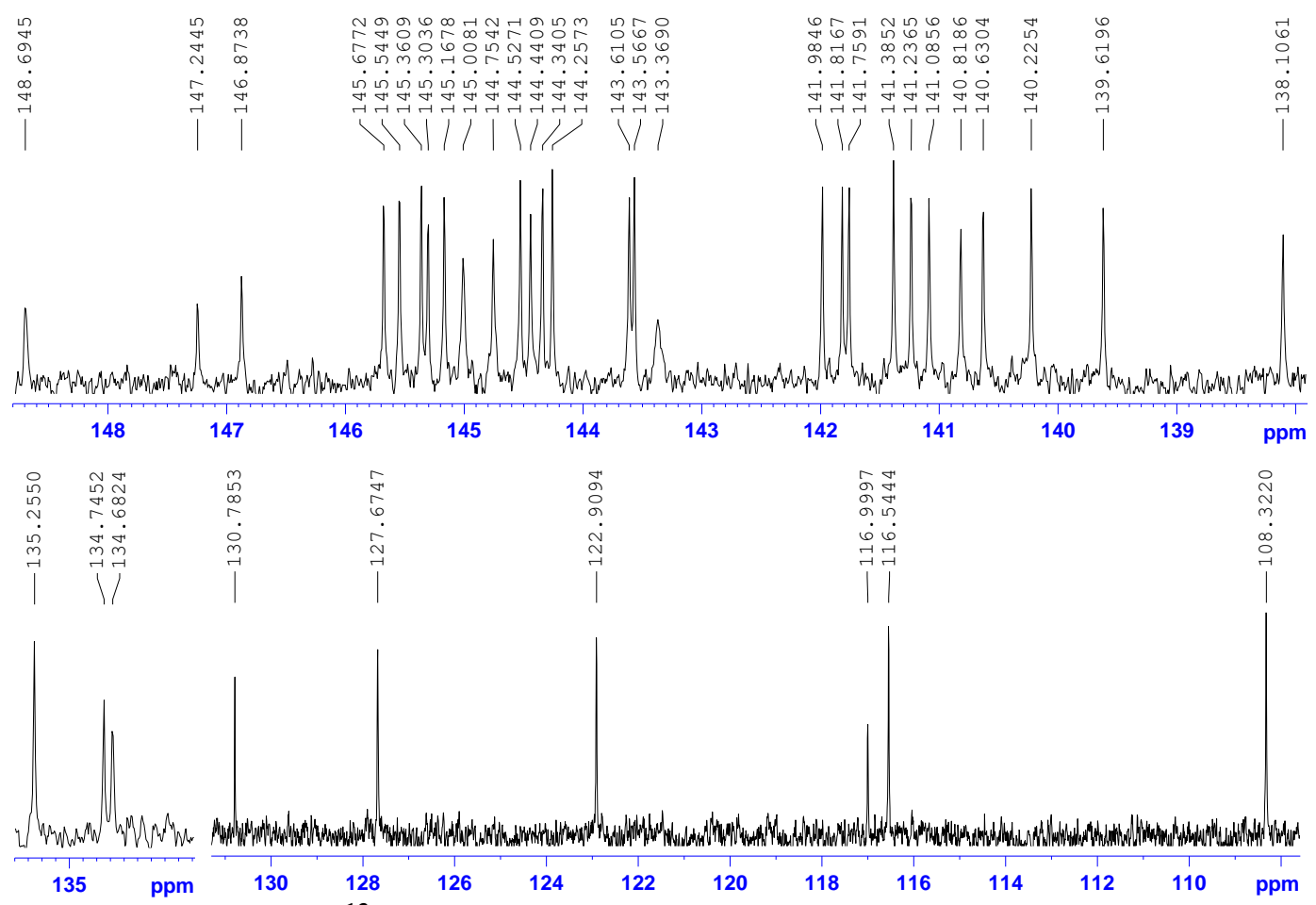

Figure S23 Expanded ${ }^{13} \mathrm{C}$ NMR (101 $\mathrm{MHz}, \mathrm{CS}_{2} / \mathrm{CDCl}_{2} \mathrm{CDCl}_{2}$ 1:2) of Compound $2 \mathrm{i}$

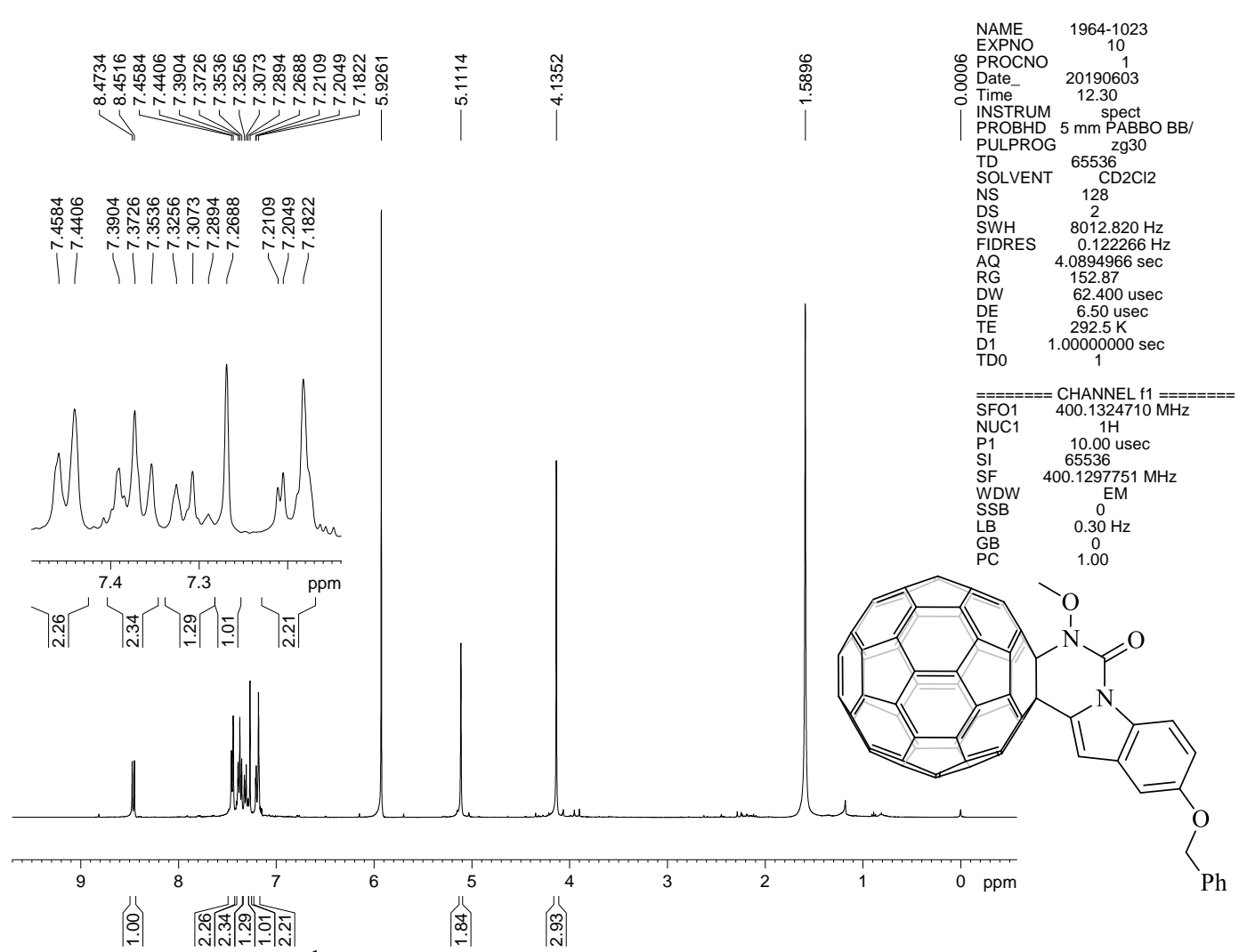

Figure $\mathrm{S24}^{1} \mathrm{H}$ NMR (400 $\left.\mathrm{MHz}, \mathrm{CDCl}_{2} \mathrm{CDCl}_{2}\right)$ of Compound $2 \mathrm{j}$ 


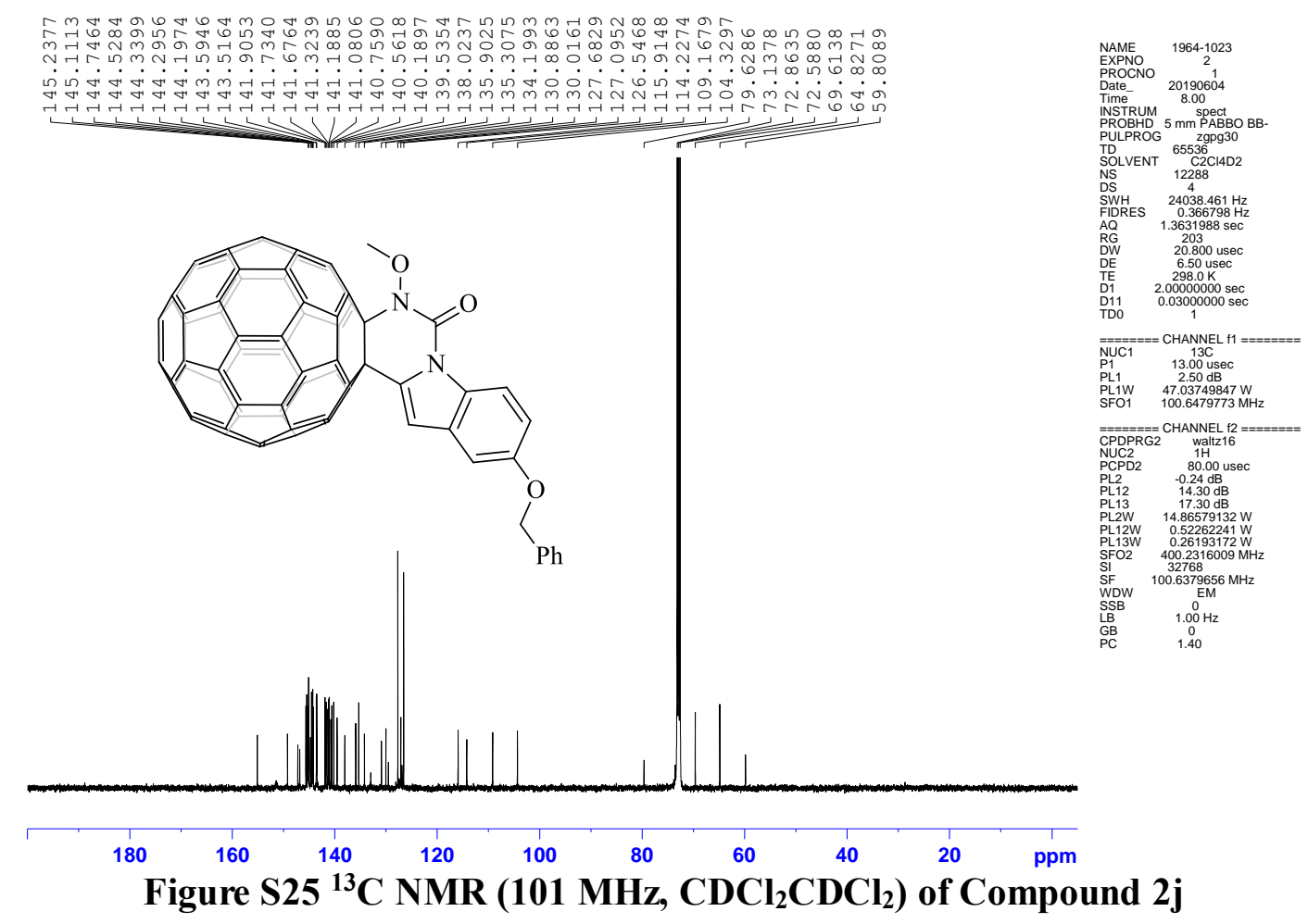

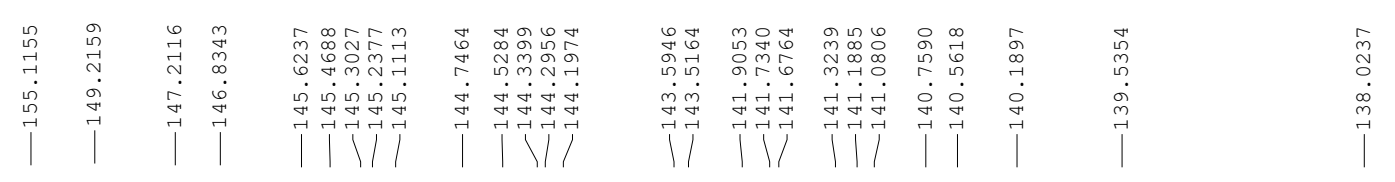
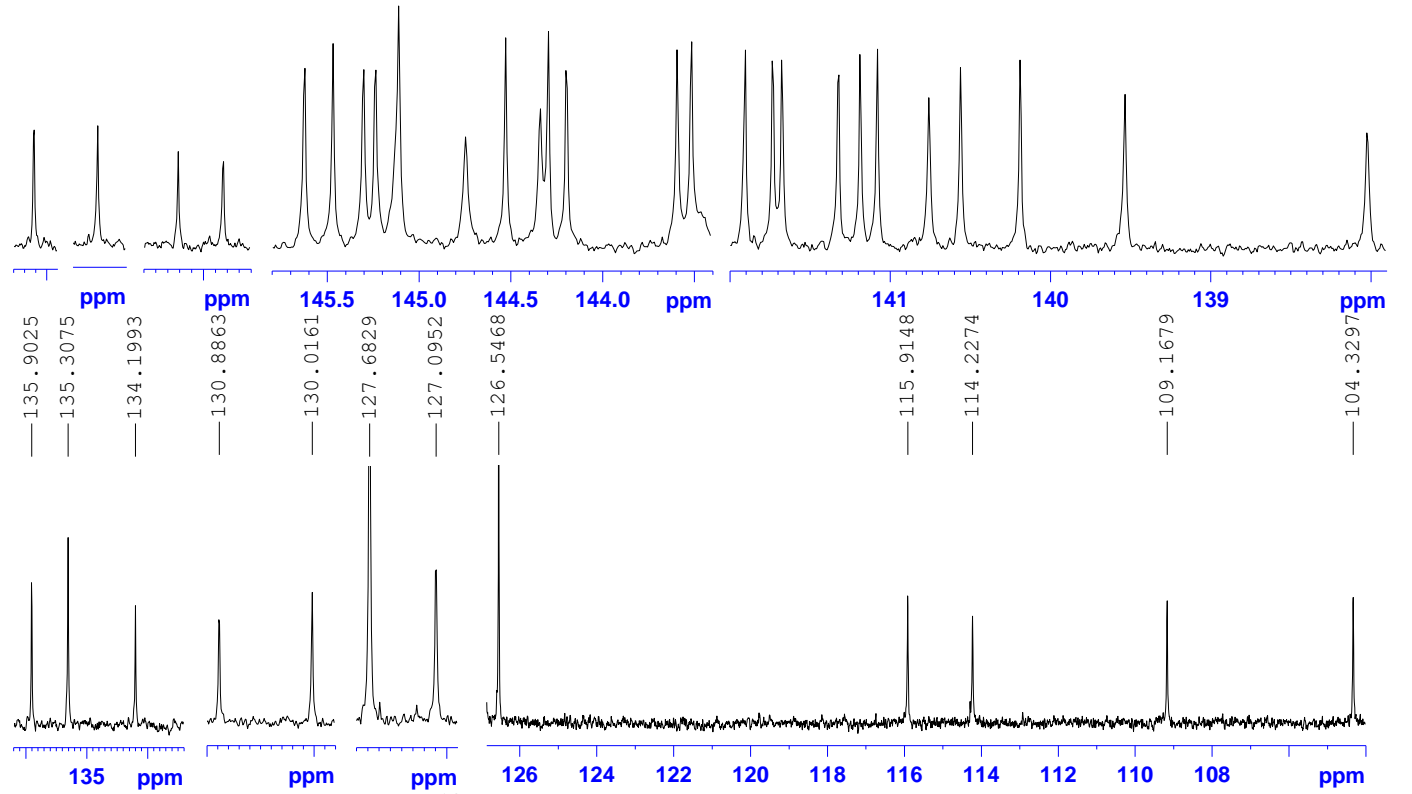

Figure S26 Expanded ${ }^{13} \mathrm{C}$ NMR (101 $\left.\mathrm{MHz}, \mathrm{CDCl}_{2} \mathrm{CDCl}_{2}\right)$ of Compound $2 \mathrm{j}$ 

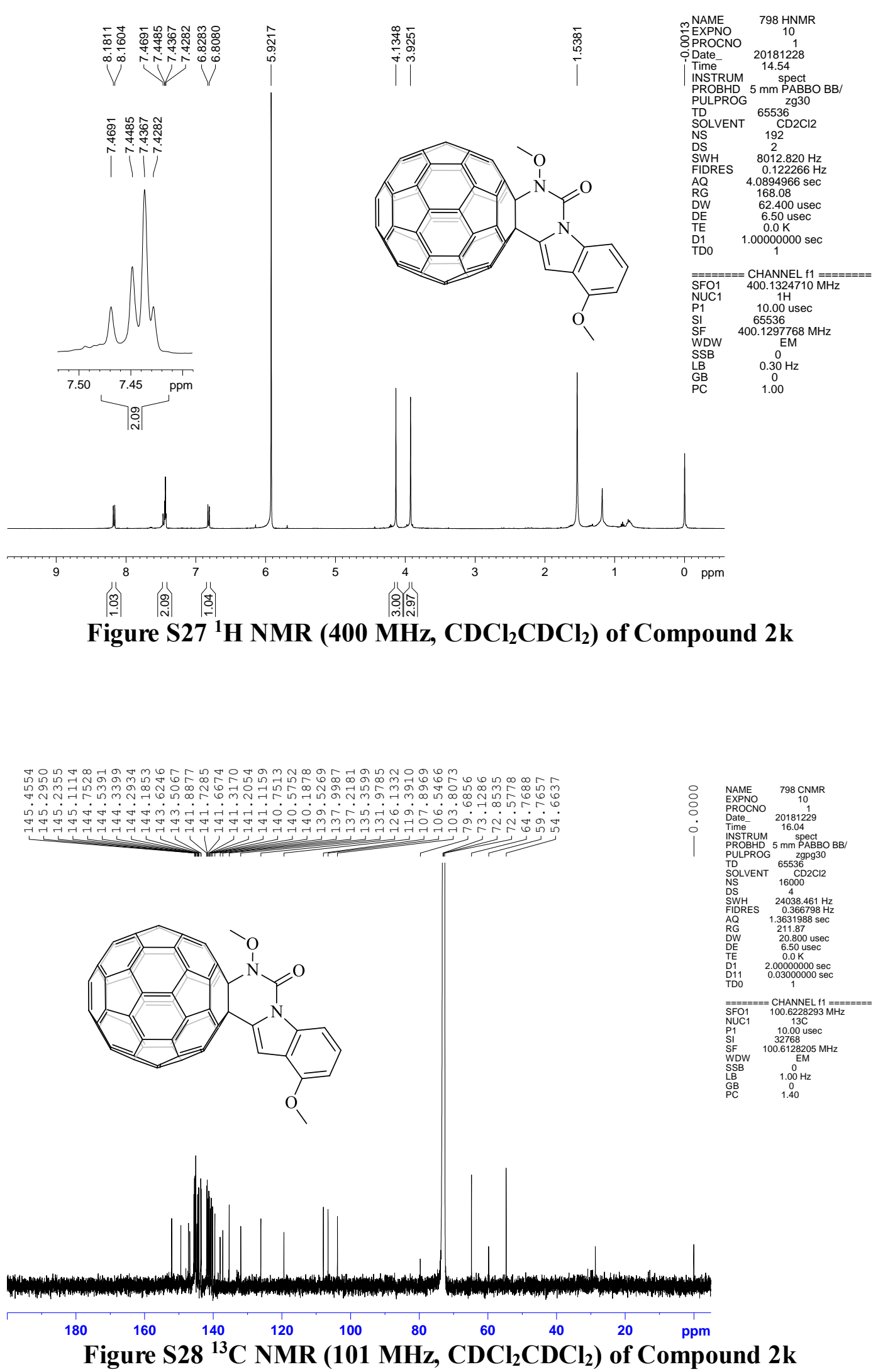

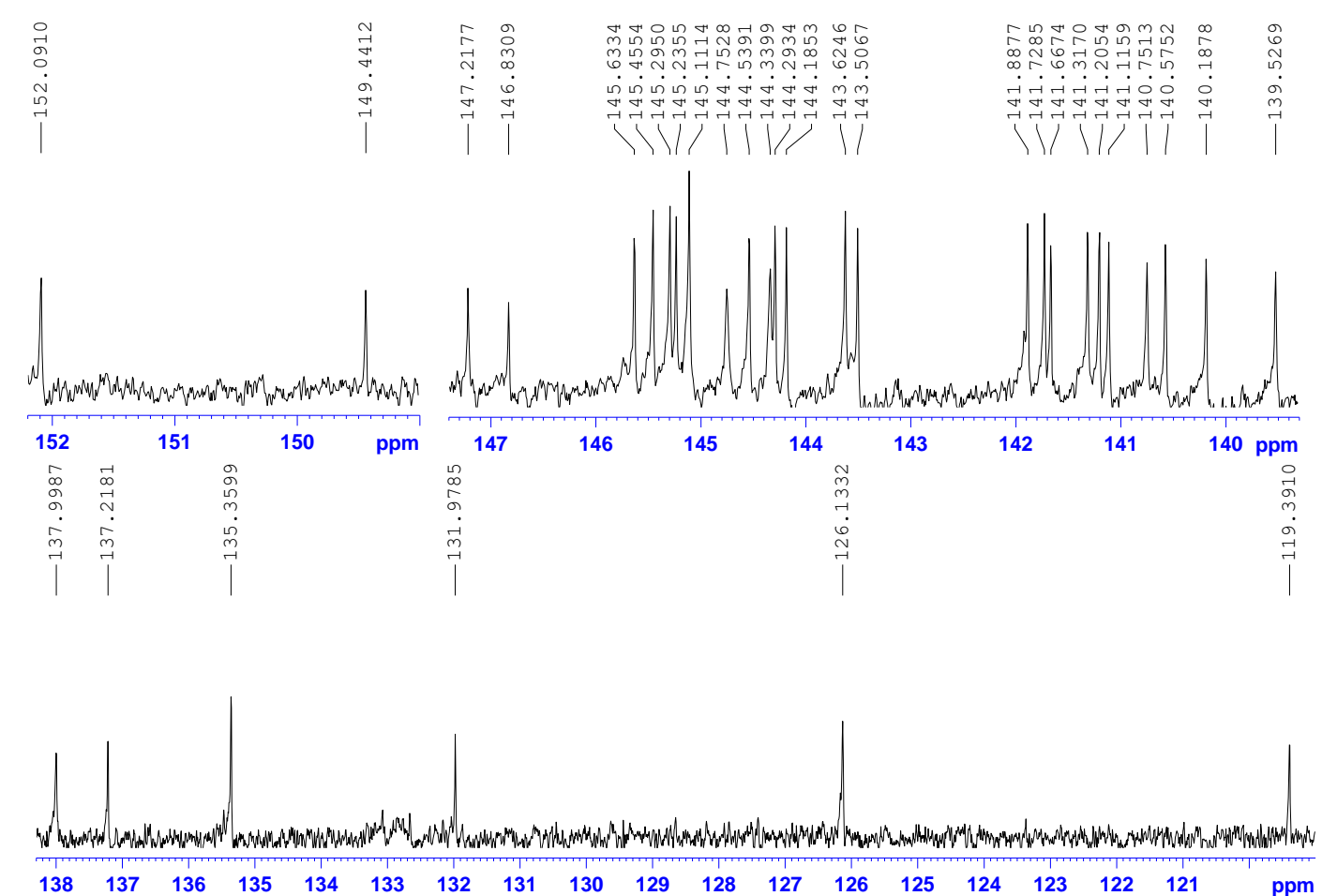
Figure S29 Expanded ${ }^{13} \mathrm{C}$ NMR (101 $\left.\mathrm{MHz}, \mathrm{CDCl}_{2} \mathrm{CDCl}_{2}\right)$ of Compound $2 \mathrm{k}$

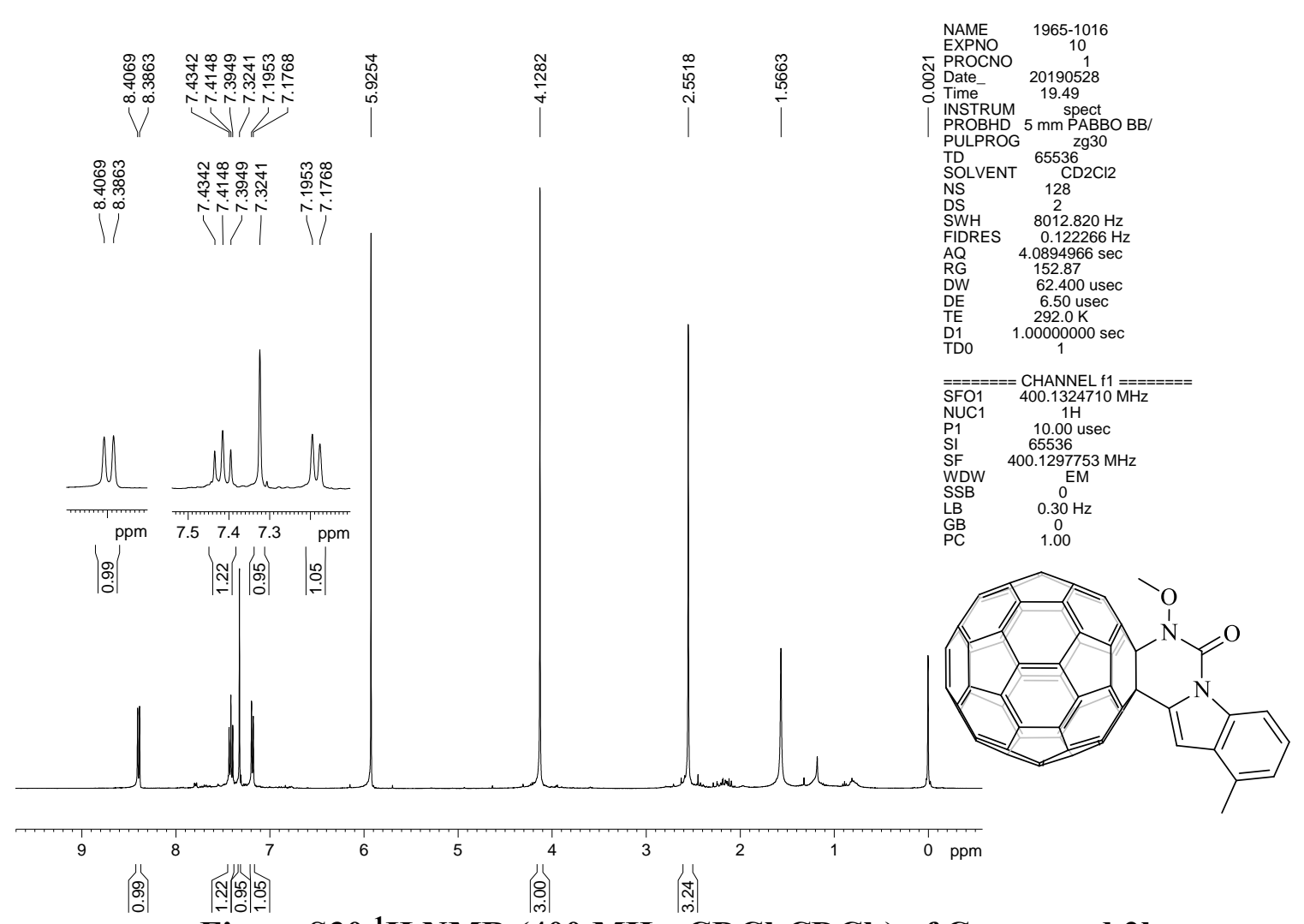

Figure $\mathrm{S30}^{1} \mathrm{H}$ NMR $\left(400 \mathrm{MHz}, \mathrm{CDCl}_{2} \mathrm{CDCl}_{2}\right)$ of Compound $2 \mathrm{I}$ 

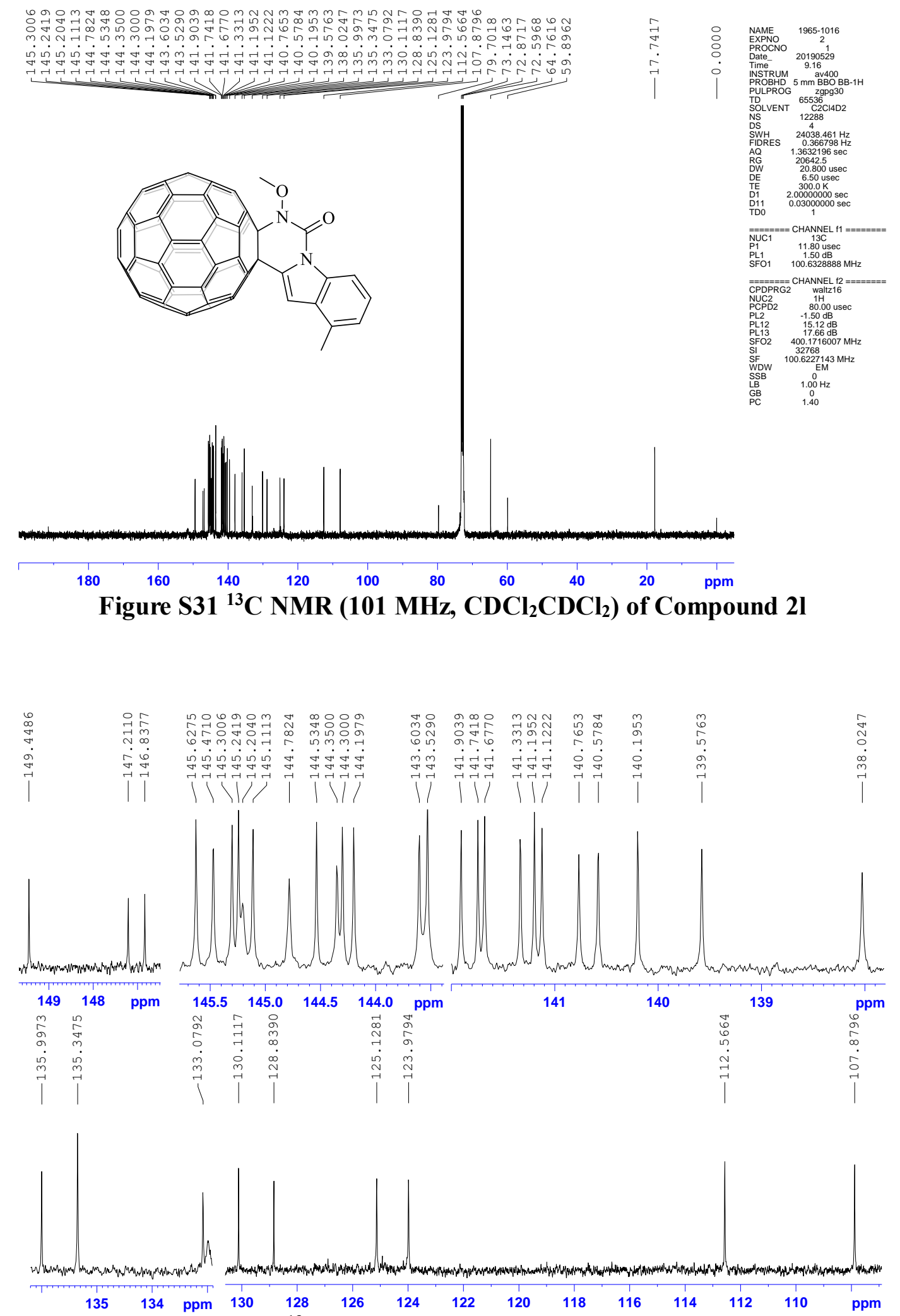

Figure S32 Expanded ${ }^{13} \mathrm{C}$ NMR (101 $\left.\mathrm{MHz}, \mathrm{CDCl}_{2} \mathrm{CDCl}_{2}\right)$ of Compound $2 \mathrm{I}$ 


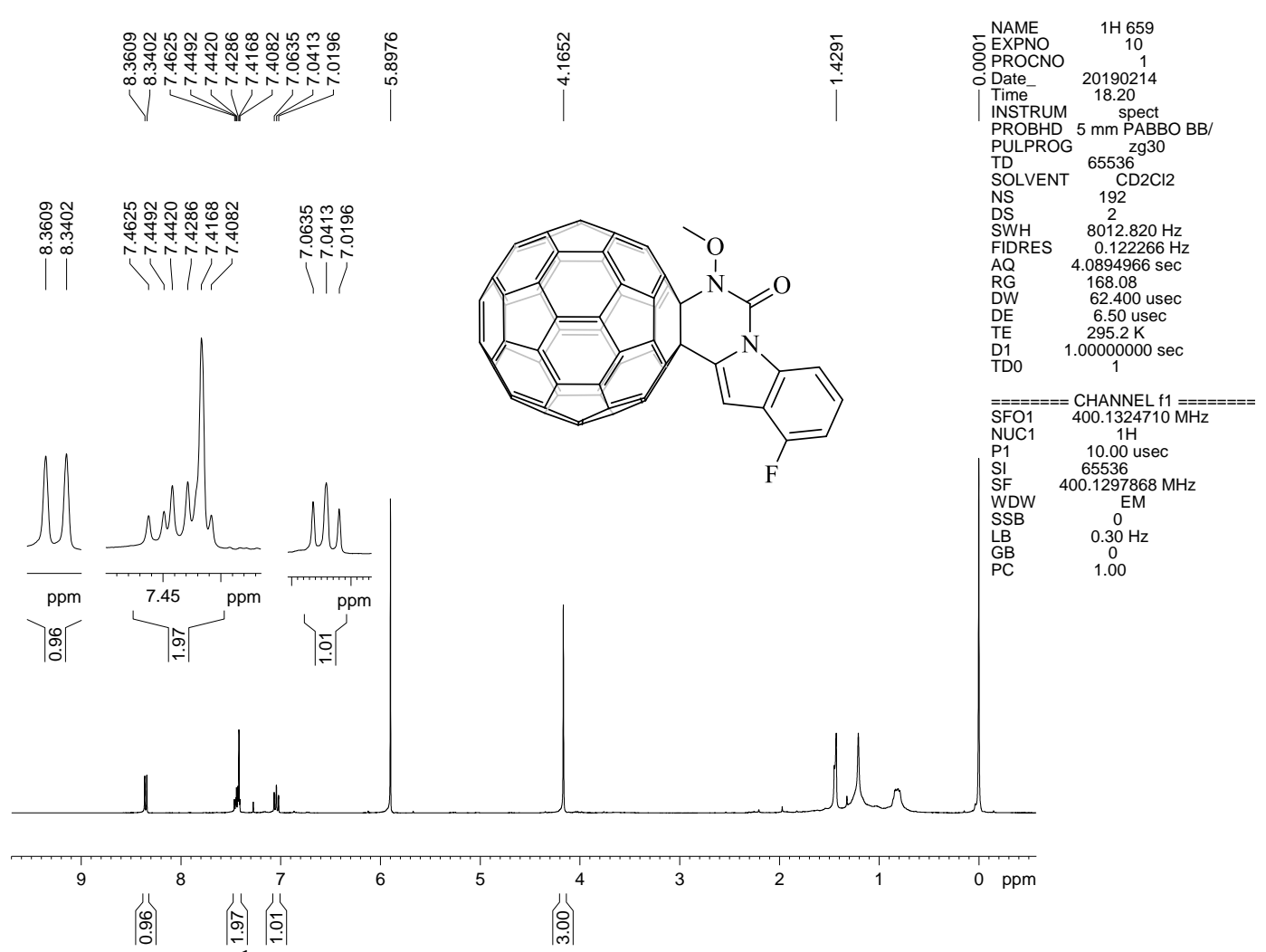

Figure S33 ${ }^{1} \mathrm{H}$ NMR (400 MHz, $\mathrm{CS}_{2} / \mathrm{CDCl}_{2} \mathrm{CDCl}_{2} 3: 1$ ) of Compound $2 \mathrm{~m}$

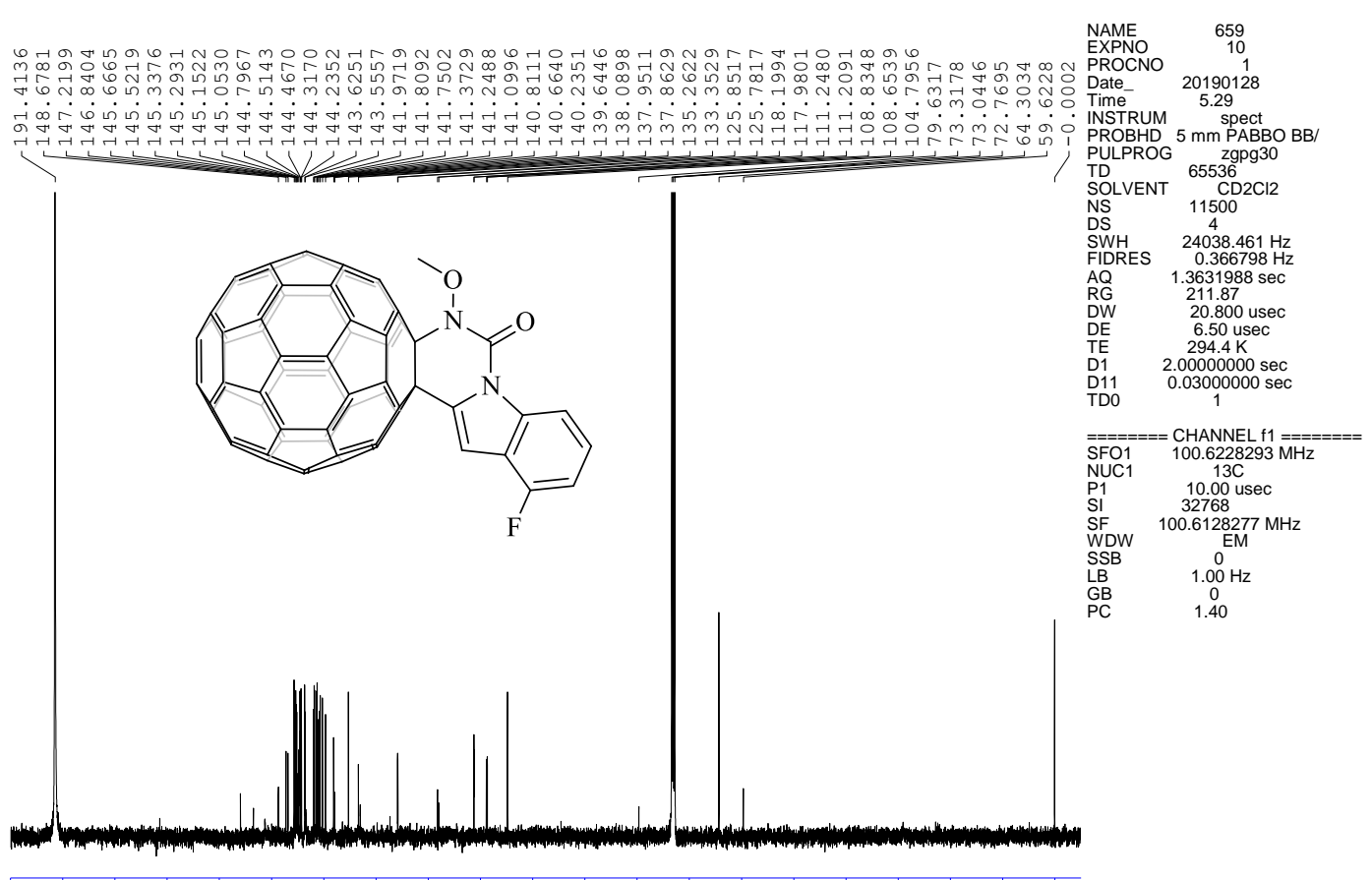

$\begin{array}{llllllllll}180 & 160 & 140 & 120 & 100 & 80 & 60 & 40 & 20 & \mathrm{ppm}\end{array}$

Figure $\mathrm{S34}^{13} \mathrm{C}$ NMR (101 MHz, $\left.\mathrm{CS}_{2} / \mathrm{CDCl}_{2} \mathrm{CDCl}_{2} 3: 1\right)$ of Compound $2 \mathrm{~m}$ 

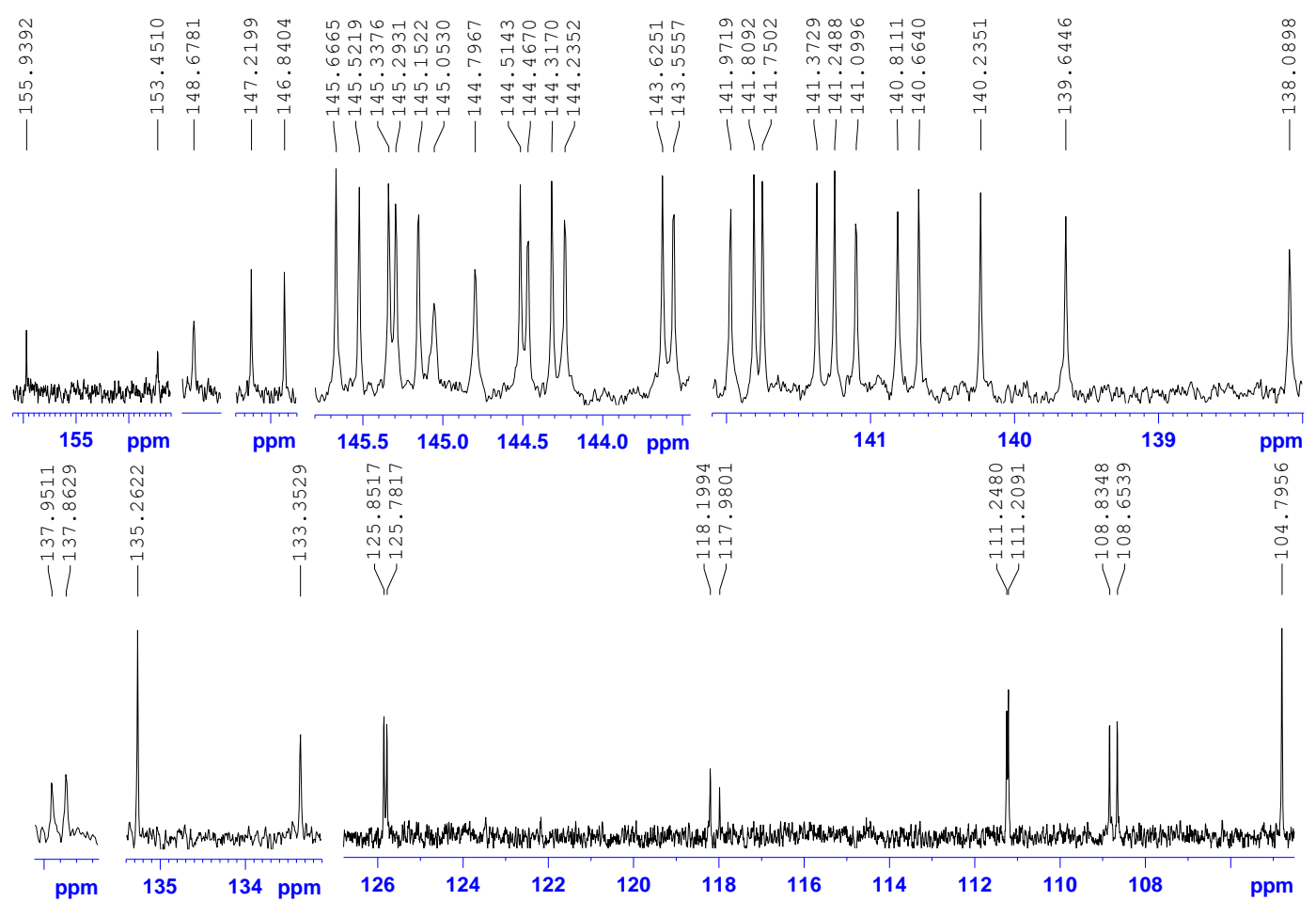

Figure S35 Expanded ${ }^{13} \mathrm{C}$ NMR (101 $\mathrm{MHz}, \mathrm{CS}_{2} / \mathrm{CDCl}_{2} \mathrm{CDCl}_{2}$ 3:1) of Compound $2 \mathrm{~m}$

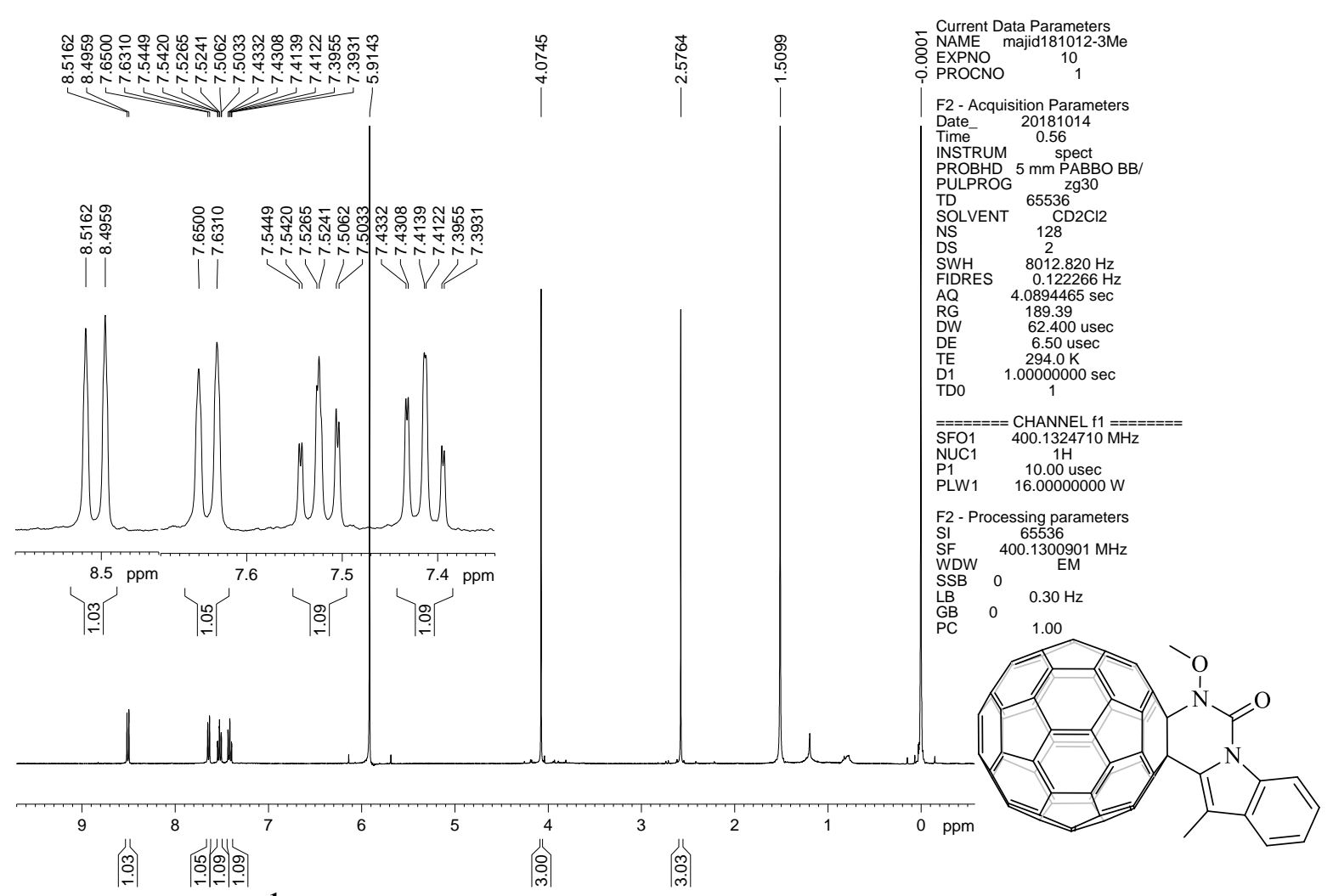

Figure S36 ${ }^{1} \mathrm{H}$ NMR (400 MHz, $\mathrm{CS}_{2} / \mathrm{CDCl}_{2} \mathrm{CDCl}_{2}$ 2:3) of Compound 2n 


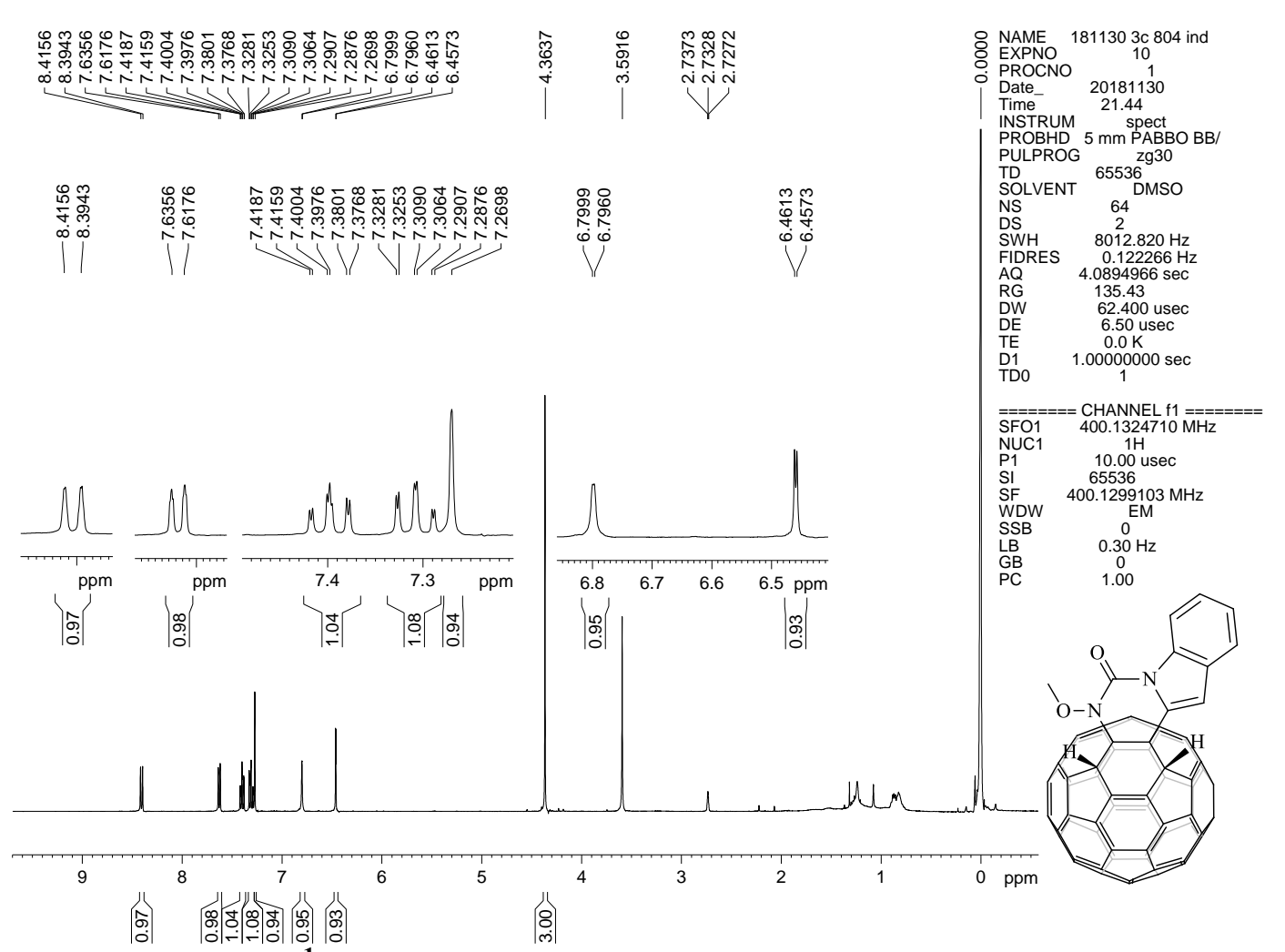

Figure S37 ${ }^{1} \mathrm{H}$ NMR (400 MHz, CS $2 / \mathrm{DMSO}_{-} d_{6}$ ) of Compound 3a

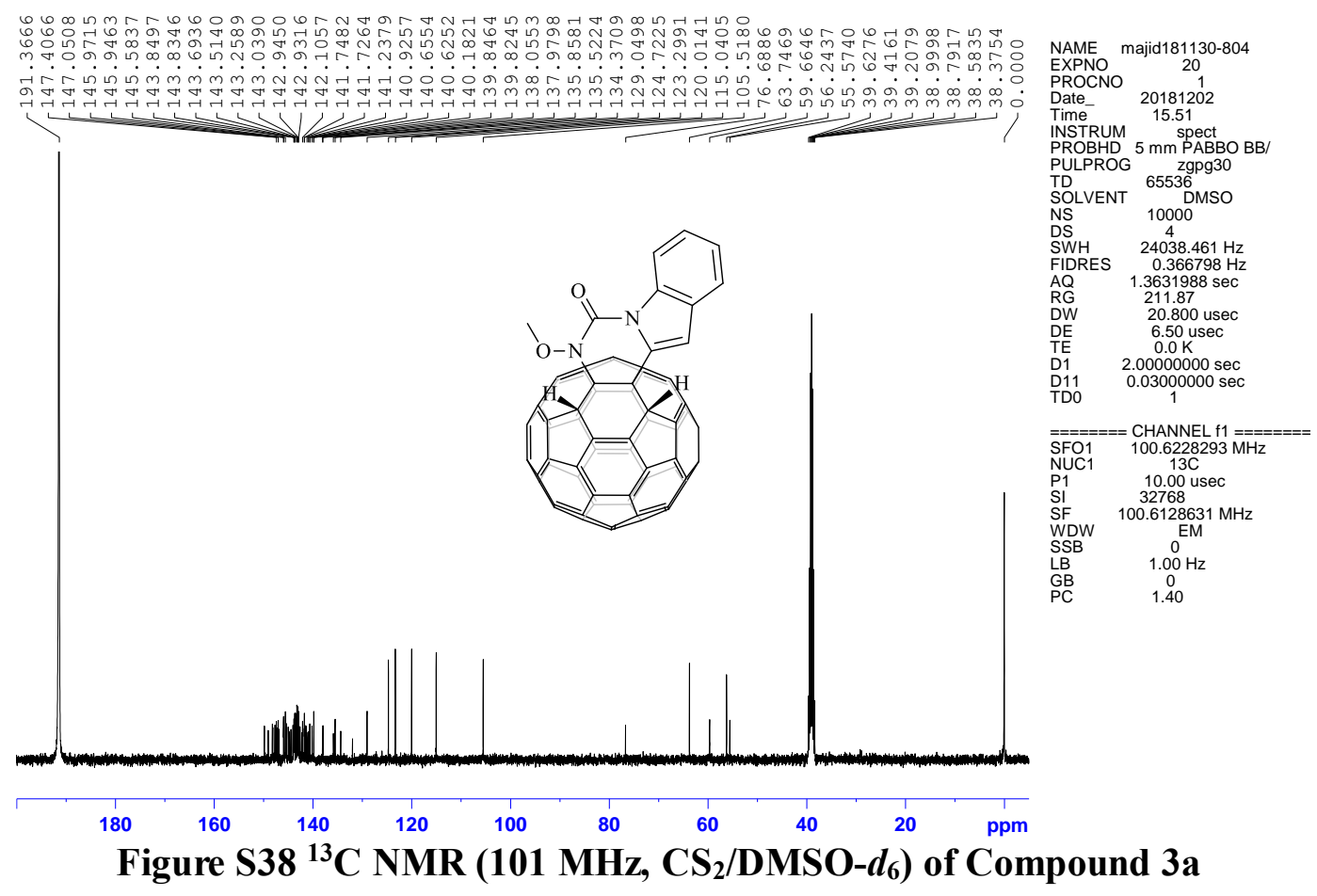



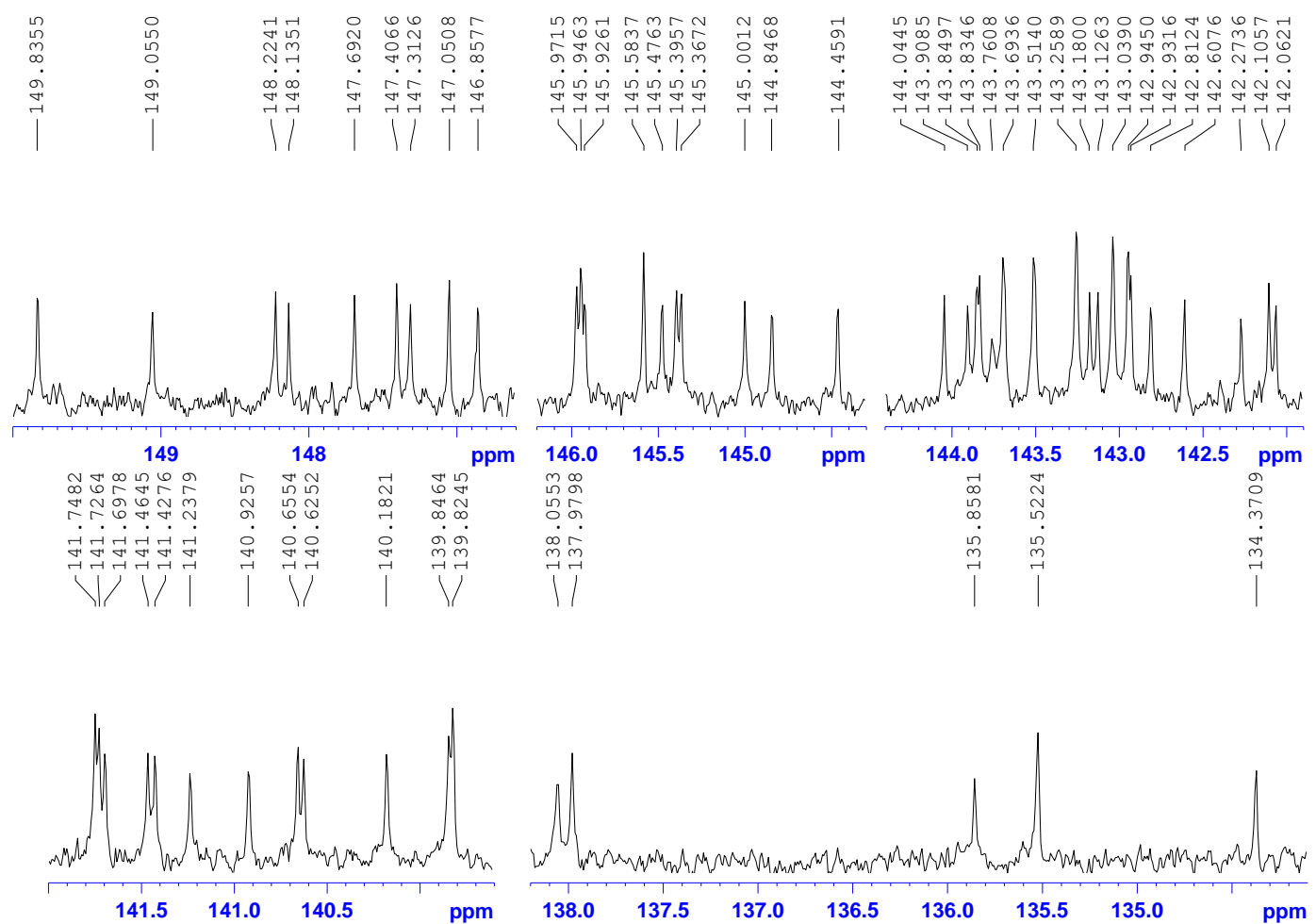

Figure S39 Expanded ${ }^{13} \mathrm{C}$ NMR (101 MHz, $\left.\mathrm{CS}_{2} / \mathrm{DMSO}-d_{6}\right)$ of Compound 3a
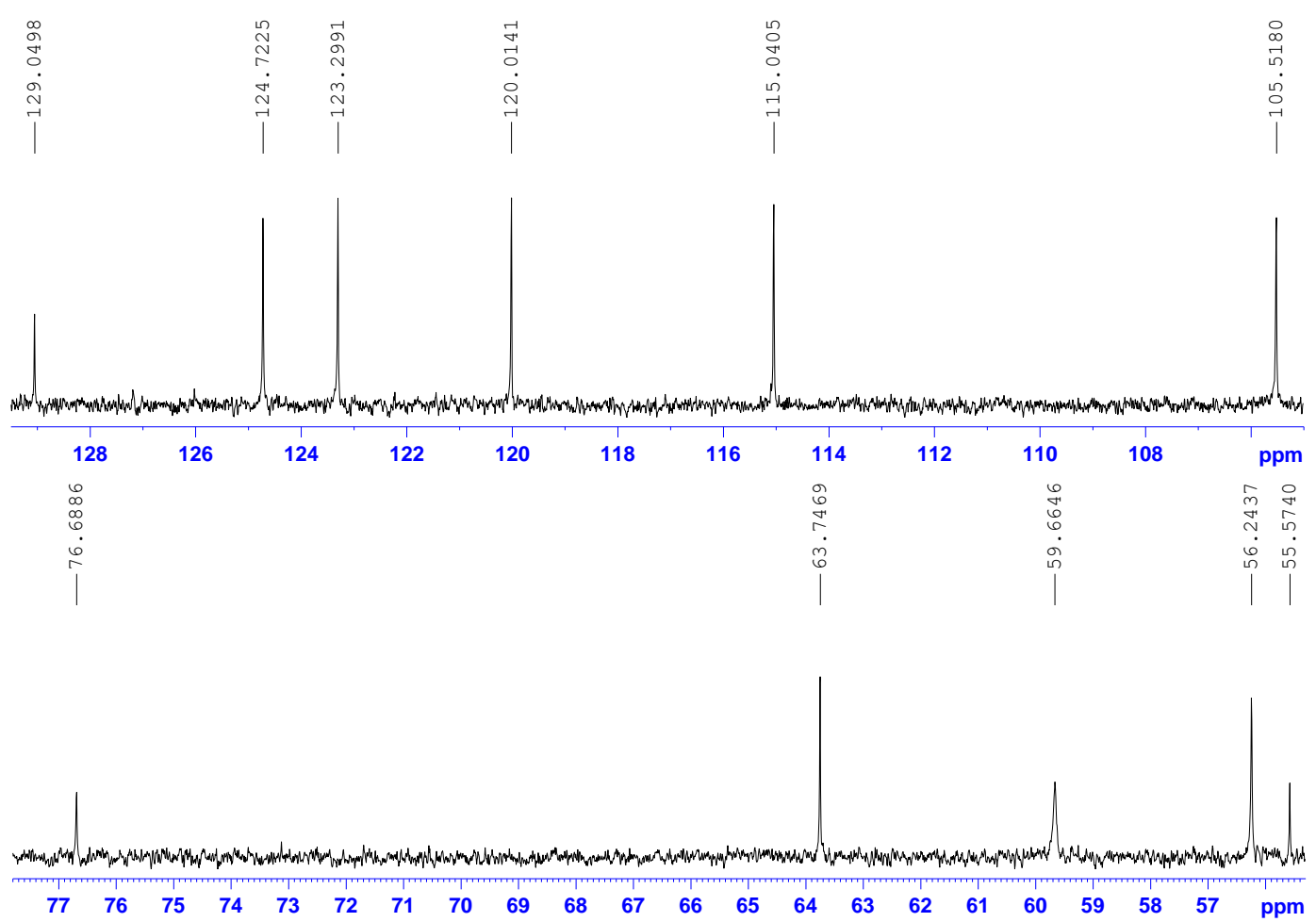

Figure S40 Expanded ${ }^{13} \mathrm{C}$ NMR (101 MHz, CS $\left.2 / D M S O-d_{6}\right)$ of Compound 3a 


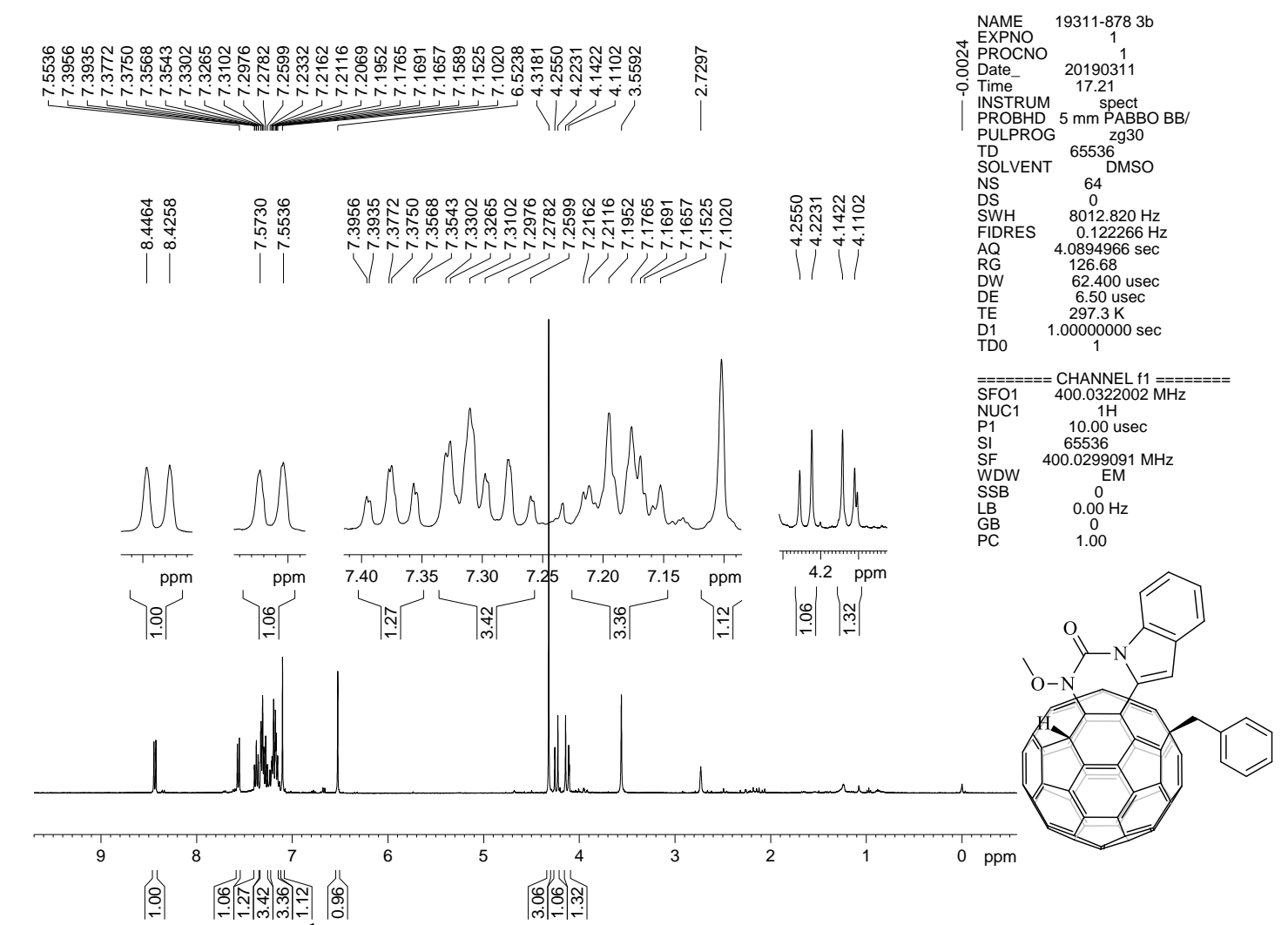

Figure S41 ${ }^{1} \mathrm{H}$ NMR (400 MHz, $\mathrm{CS}_{2} / \mathrm{DMSO}-d_{6}$ ) of Compound 3b

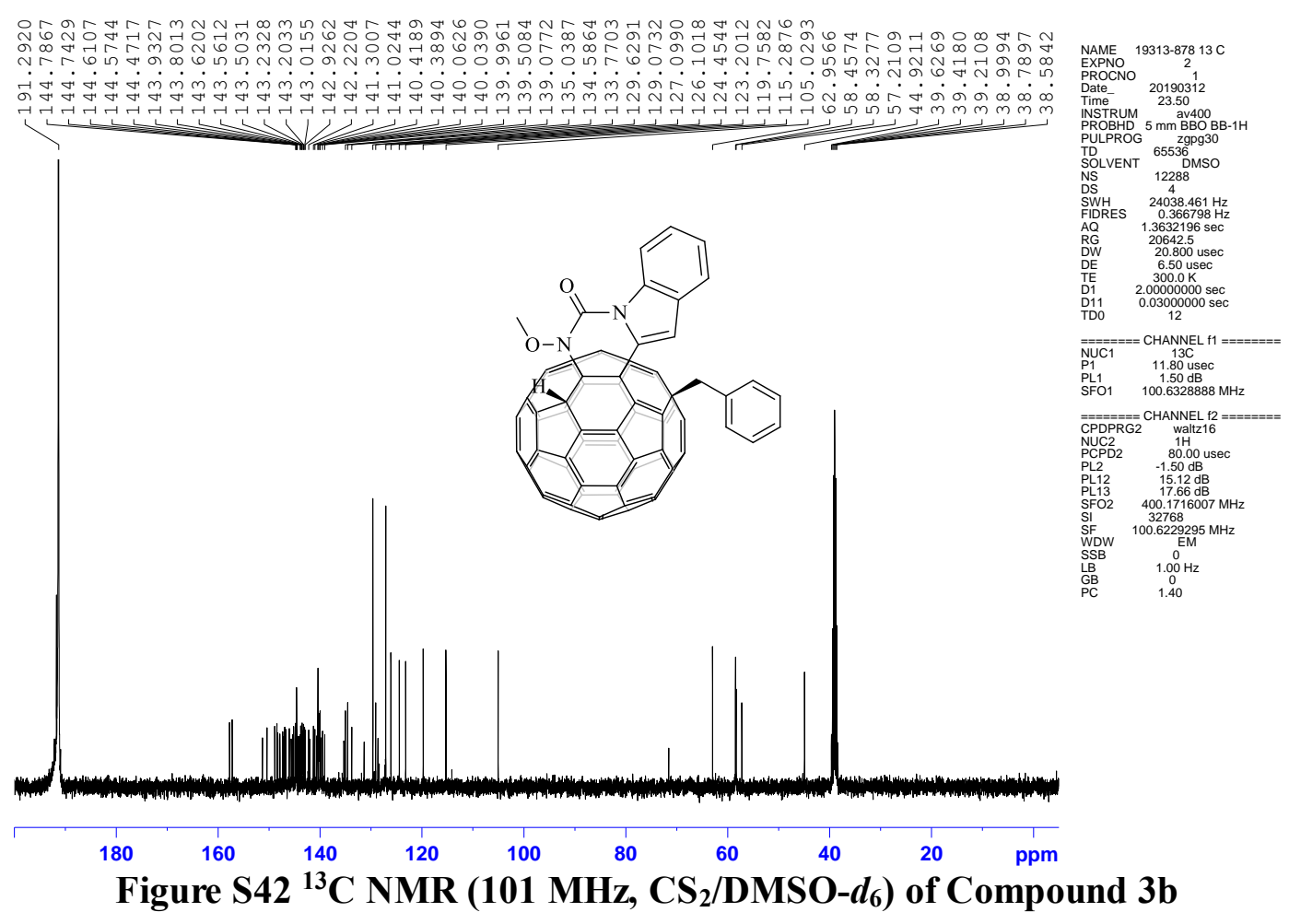



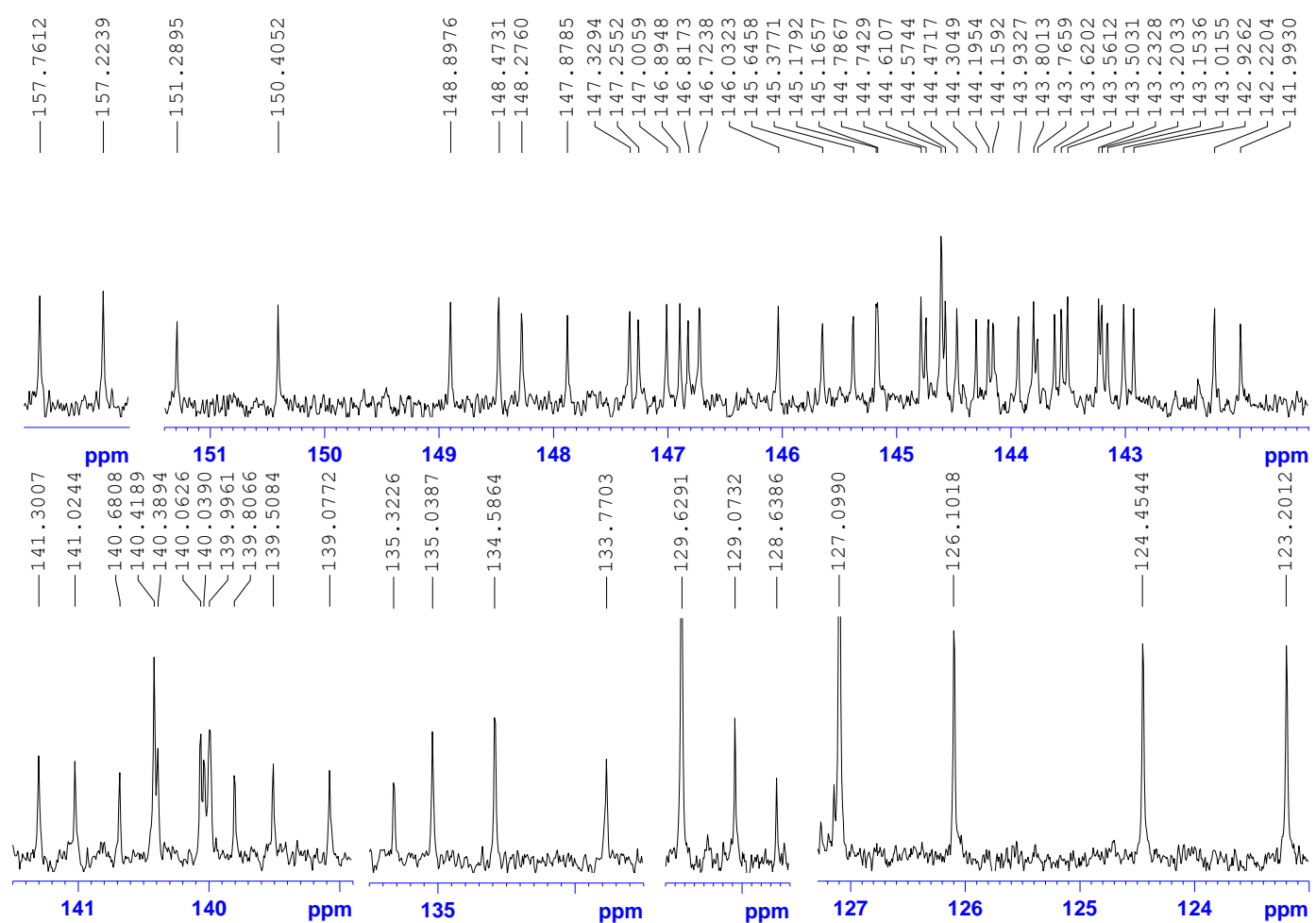

Figure S43 Expanded ${ }^{13} \mathrm{C}$ NMR (101 MHz, CS $2 / \mathrm{DMSO}^{\left.-d_{6}\right)}$ of Compound 3b

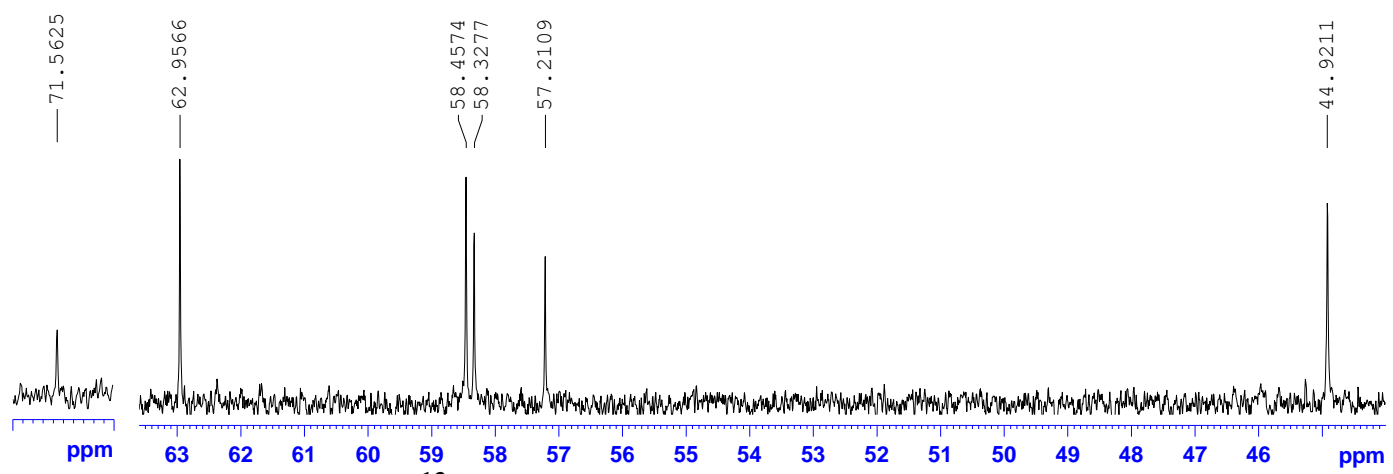

Figure S44 Expanded ${ }^{13} \mathrm{C}$ NMR (101 MHz, CS $\left./ \mathrm{DMSO}_{2} d_{6}\right)$ of Compound 3b 


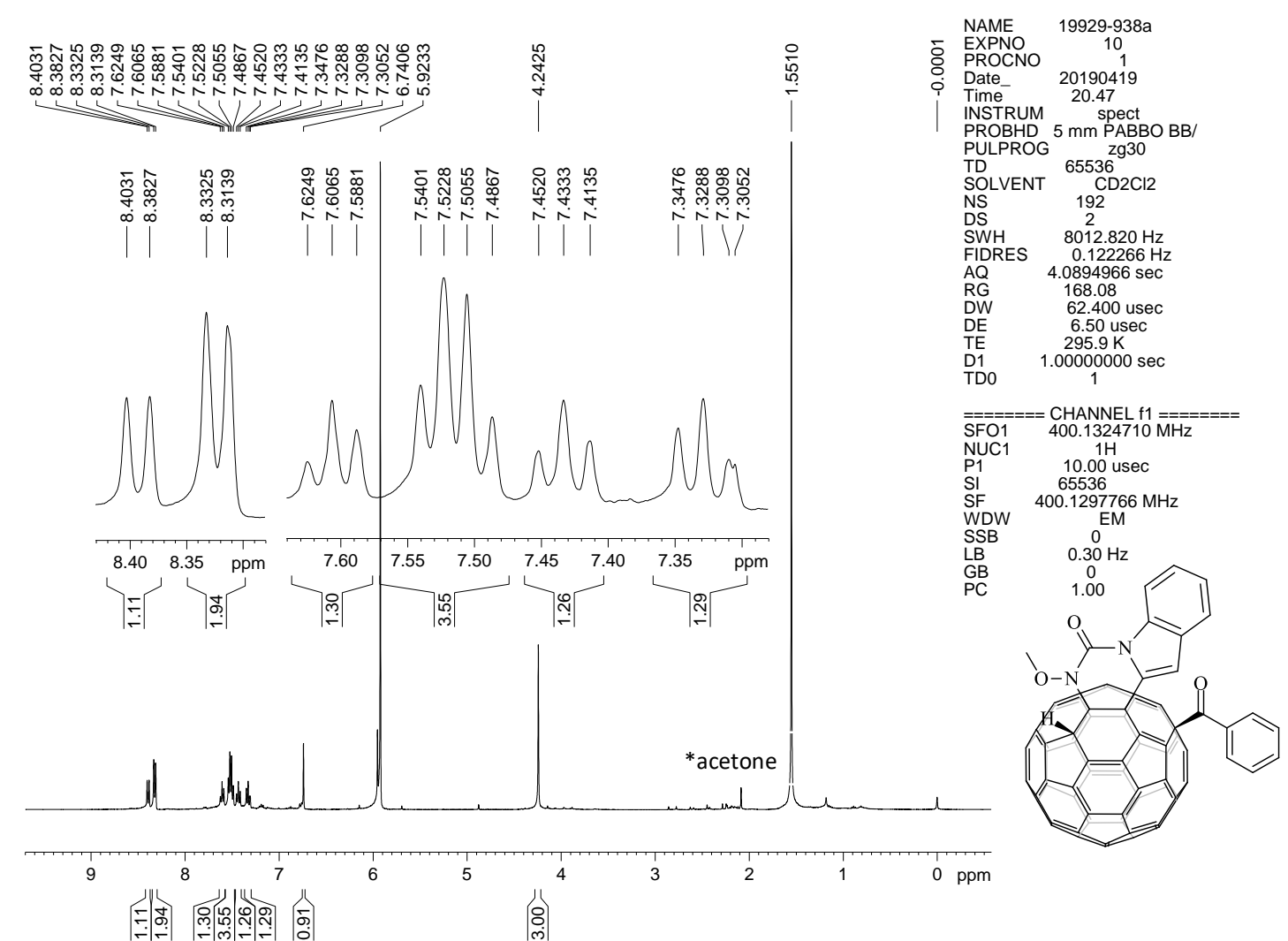

Figure S45 ${ }^{1} \mathrm{H}$ NMR (400 $\left.\mathrm{MHz}, \mathrm{CDCl}_{2} \mathrm{CDCl}_{2}\right)$ of Compound $3 \mathrm{c}$

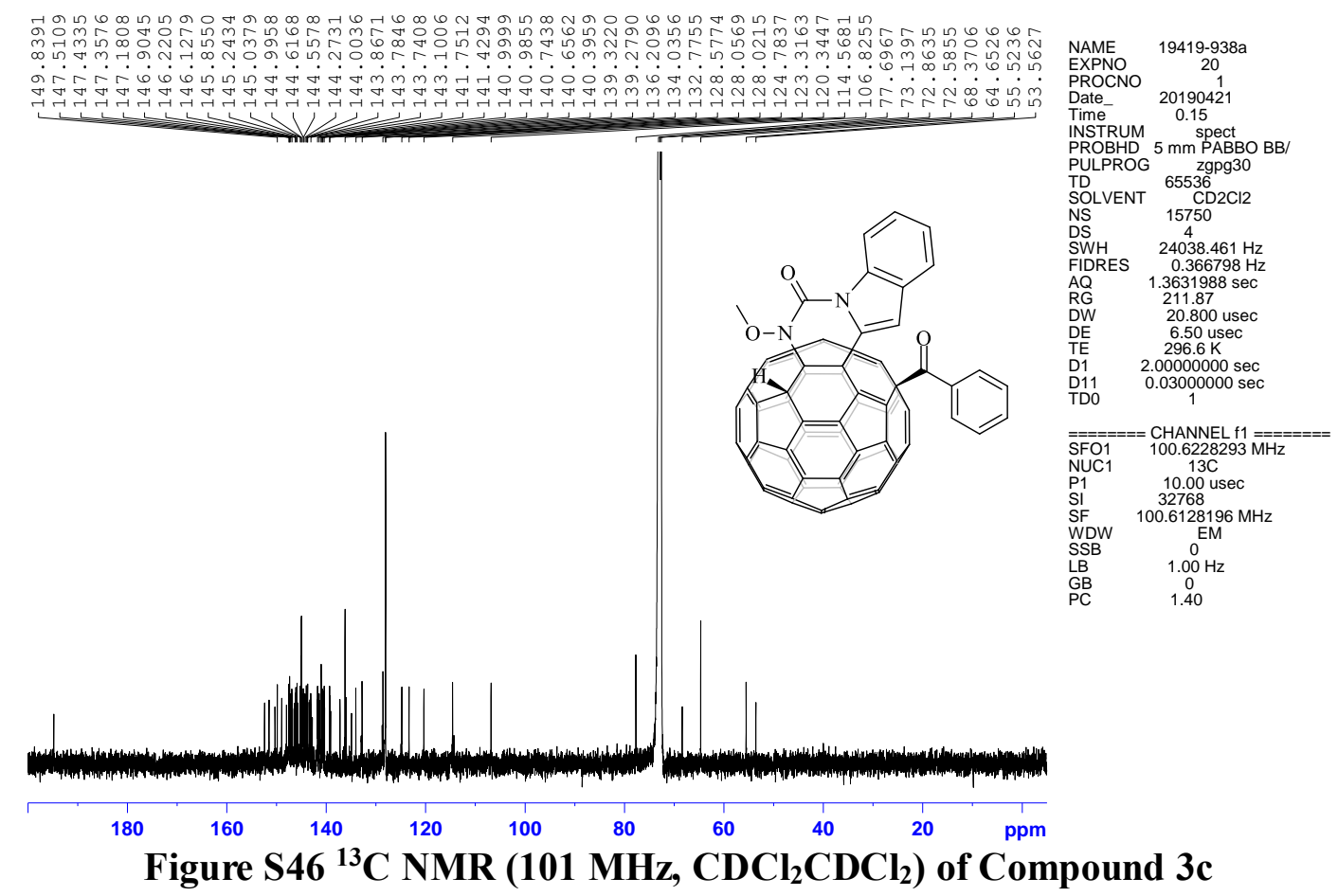




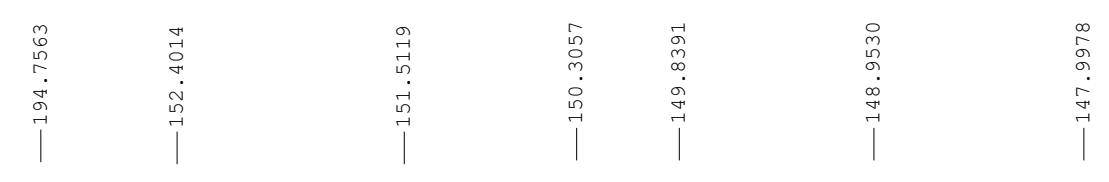
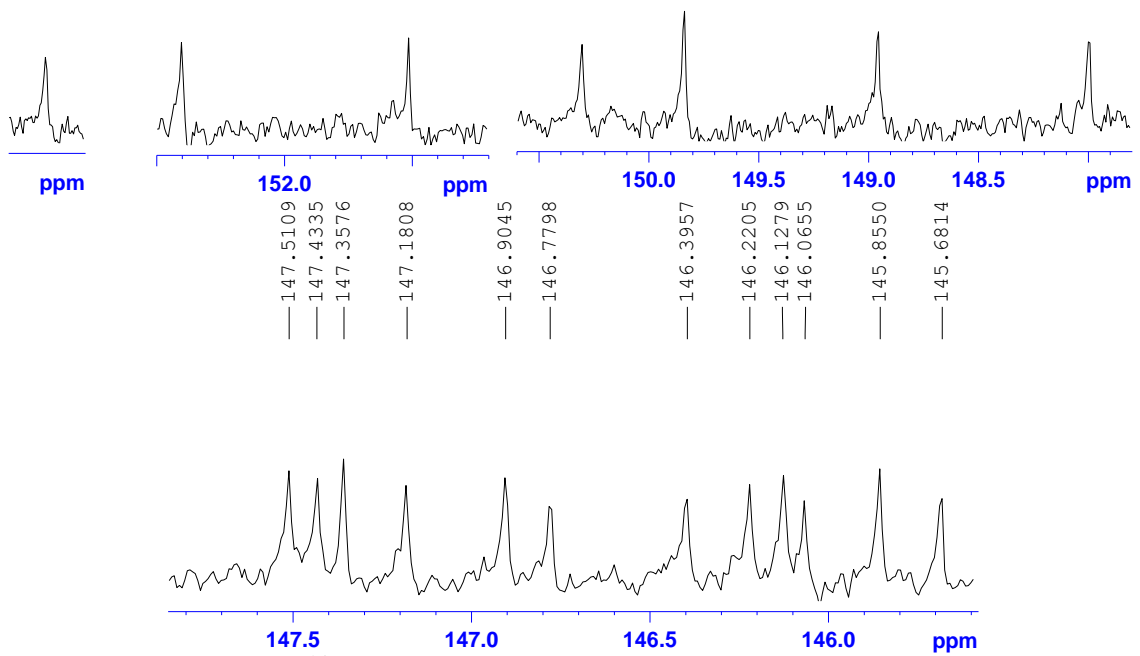

Figure S47 Expanded ${ }^{13} \mathrm{C}$ NMR (101 MHz, $\left.\mathrm{CDCl}_{2} \mathrm{CDCl}_{2}\right)$ of Compound 3c
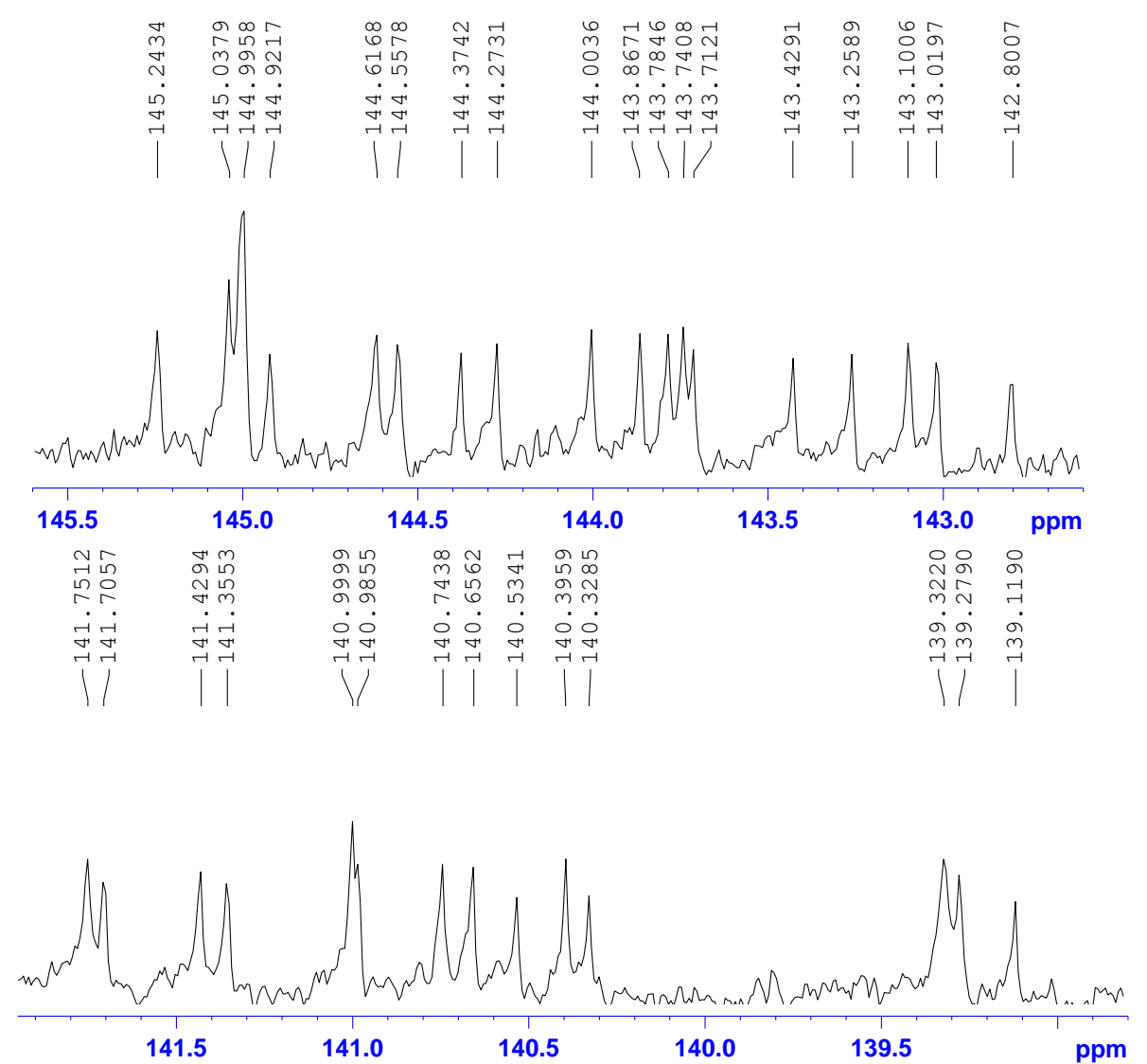

Figure S48 Expanded ${ }^{13} \mathrm{C}$ NMR (101 MHz, $\left.\mathrm{CDCl}_{2} \mathrm{CDCl}_{2}\right)$ of Compound 3c 

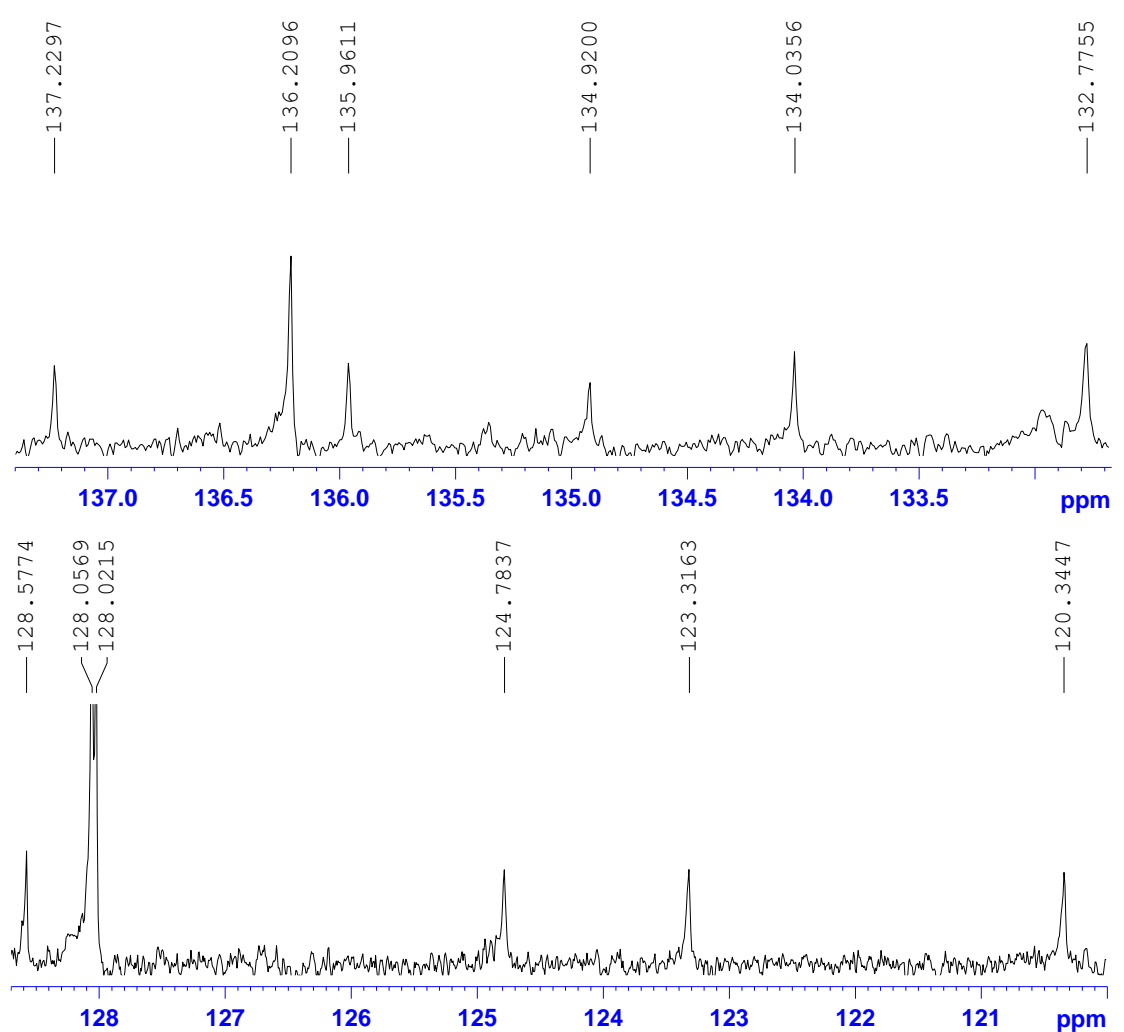

Figure S49 Expanded ${ }^{13} \mathrm{C}$ NMR (101 $\left.\mathrm{MHz}, \mathrm{CDCl}_{2} \mathrm{CDCl}_{2}\right)$ of Compound $3 \mathrm{c}$

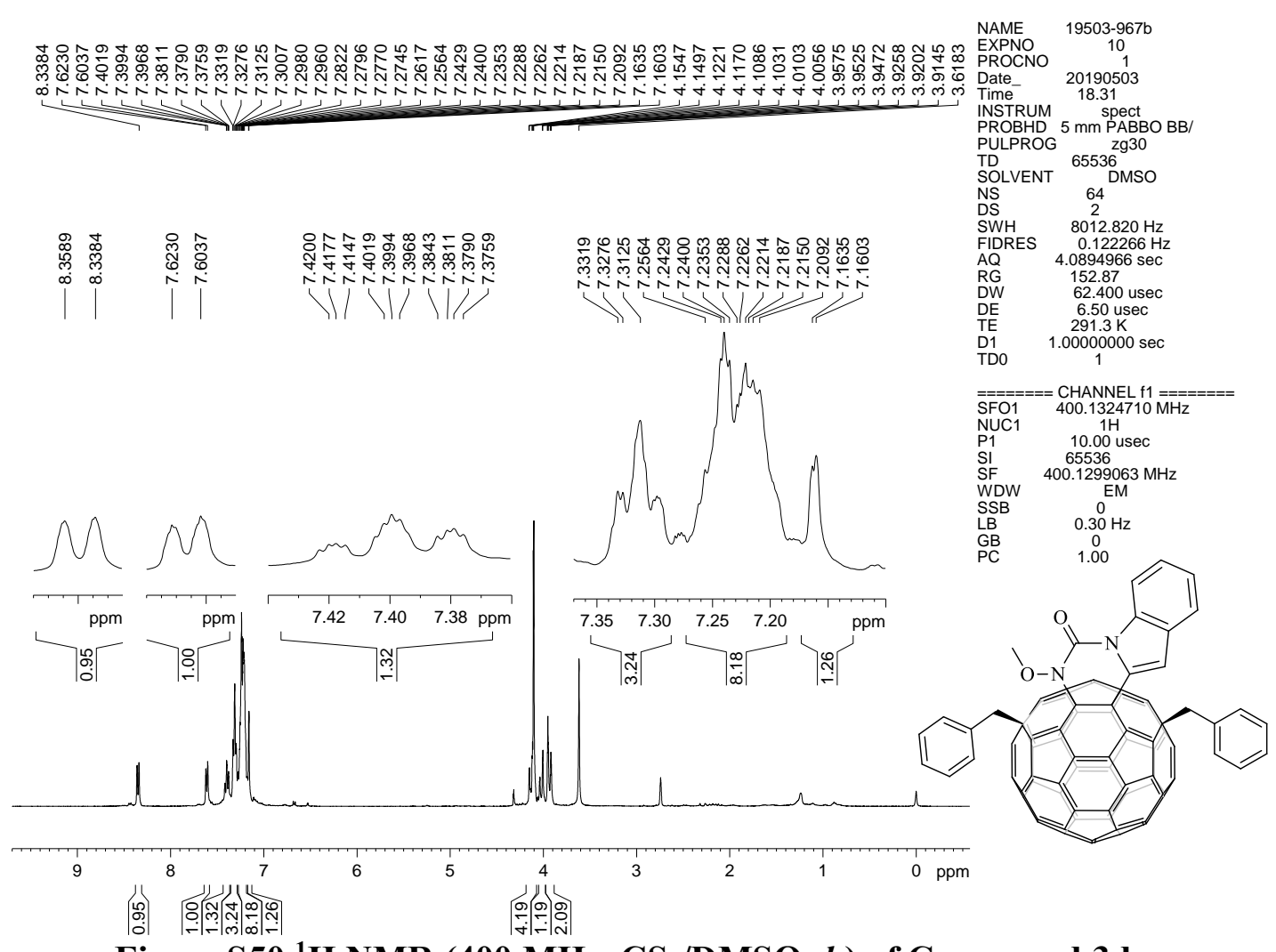

Figure S50 ${ }^{1} \mathrm{H}$ NMR (400 MHz, CS $2 / \mathrm{DMSO}_{-} d_{6}$ ) of Compound 3d 

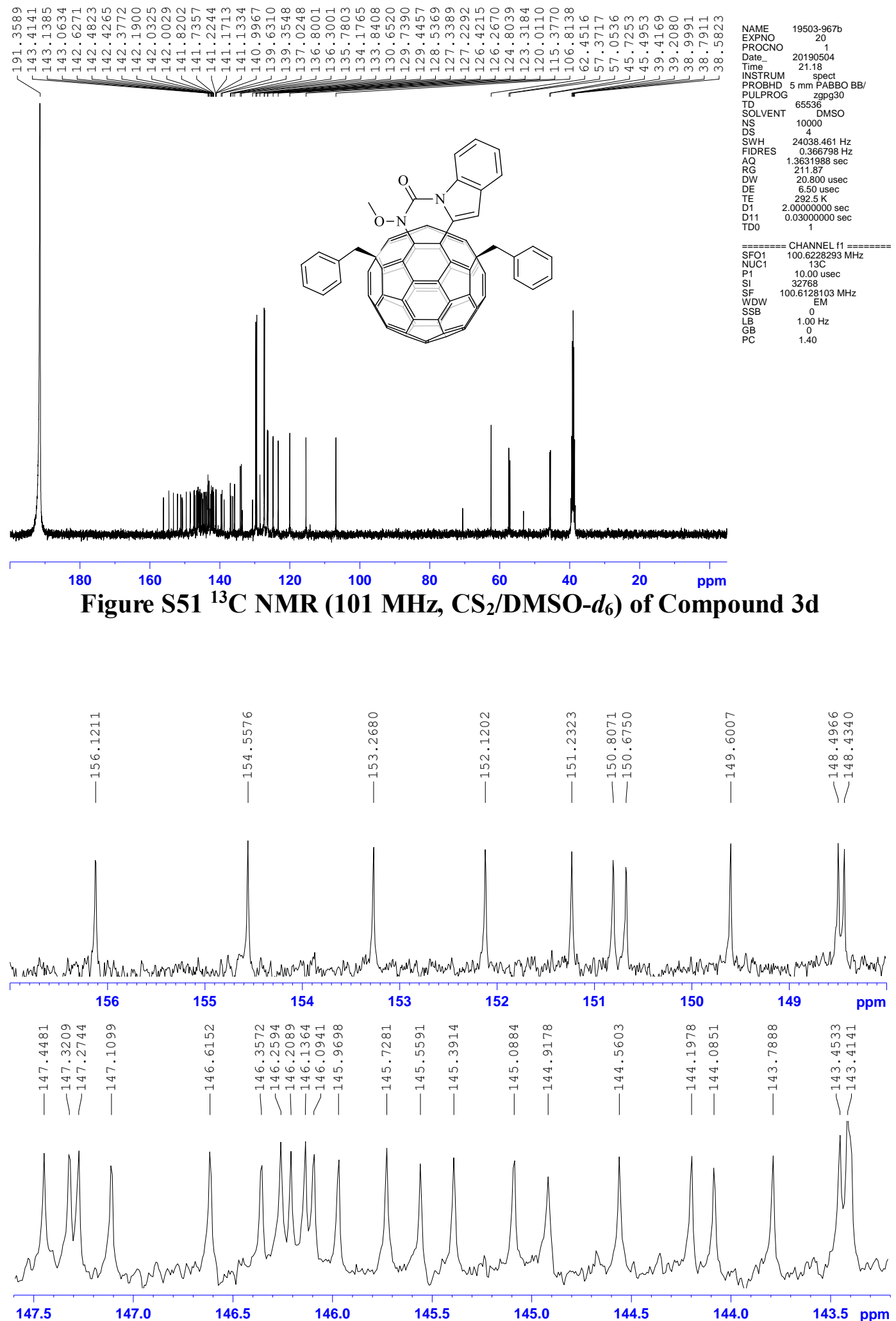

Figure S52 Expanded ${ }^{13} \mathrm{C}$ NMR (101 MHz, CS $\left.2 / D M S O-d 6\right)$ of Compound 3d 


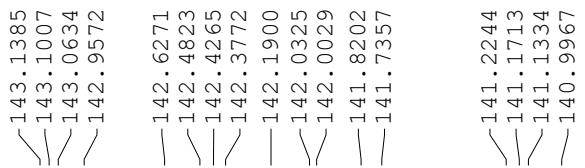
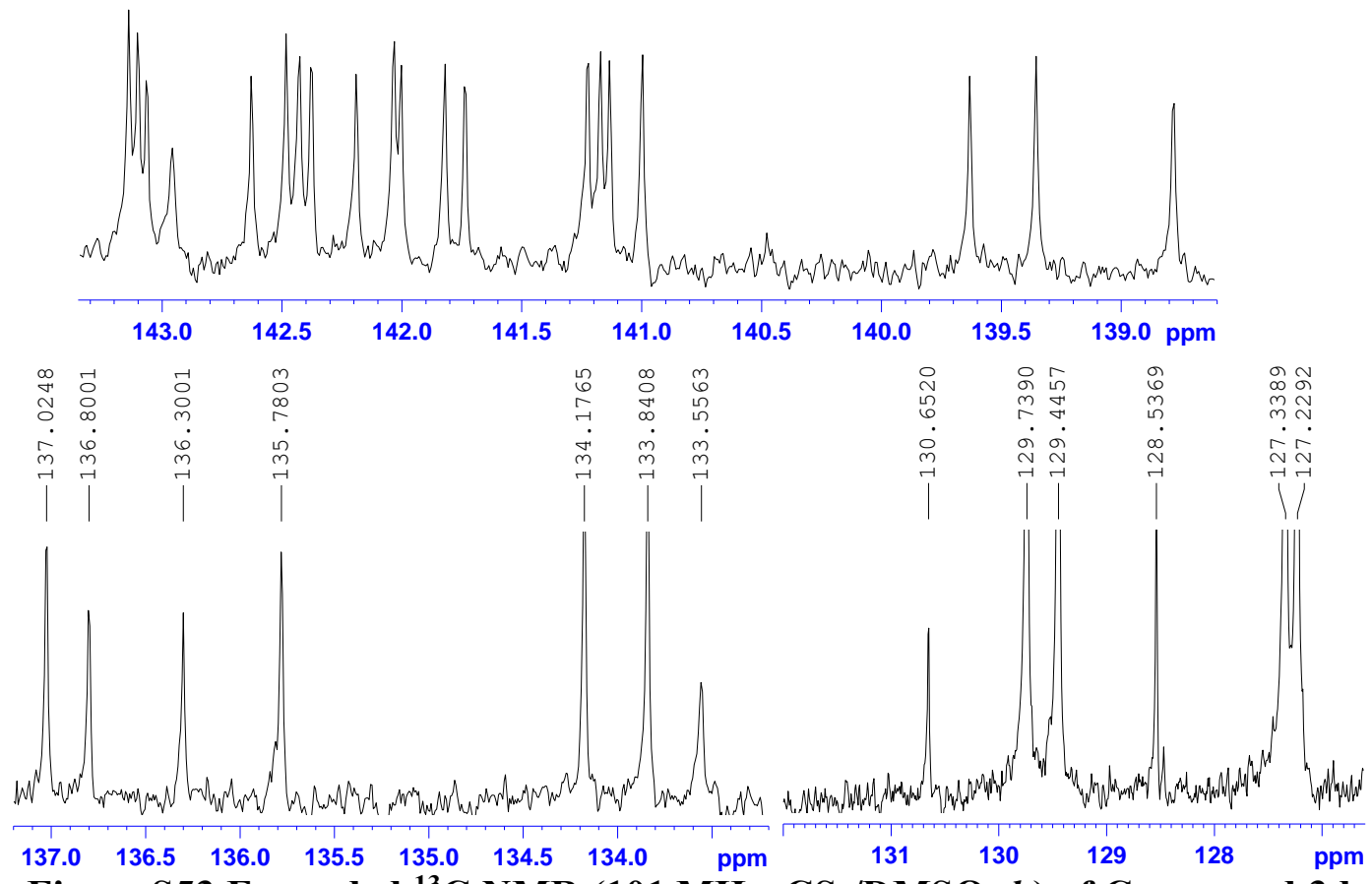

Figure S53 Expanded ${ }^{13} \mathrm{C}$ NMR (101 MHz, CS $/$ DMSO- $\left.d_{6}\right)$ of Compound 3d
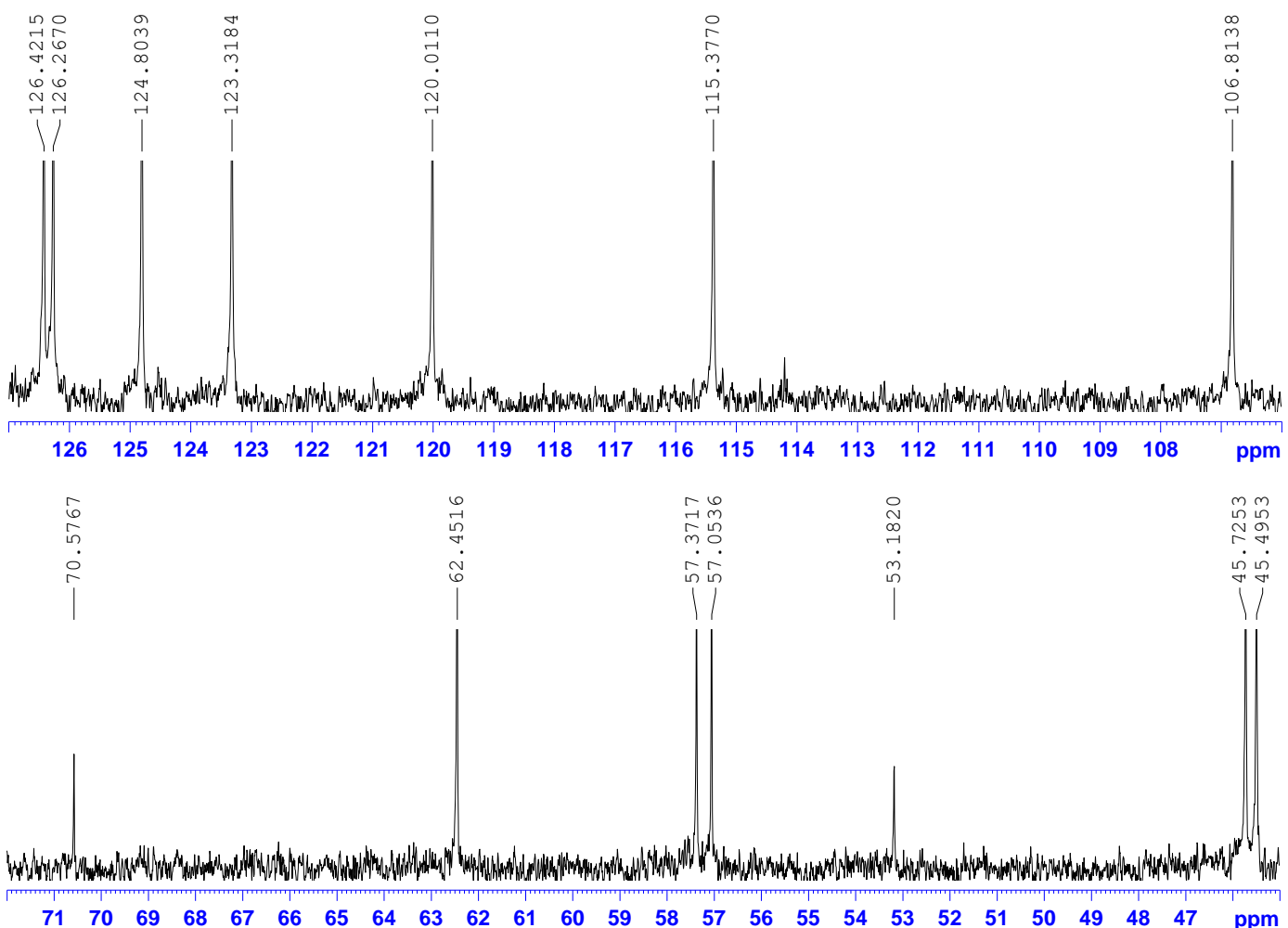

Figure S54 Expanded ${ }^{13} \mathrm{C}$ NMR (101 MHz, CS $\left.2 / D M S O-d_{6}\right)$ of Compound 3d 


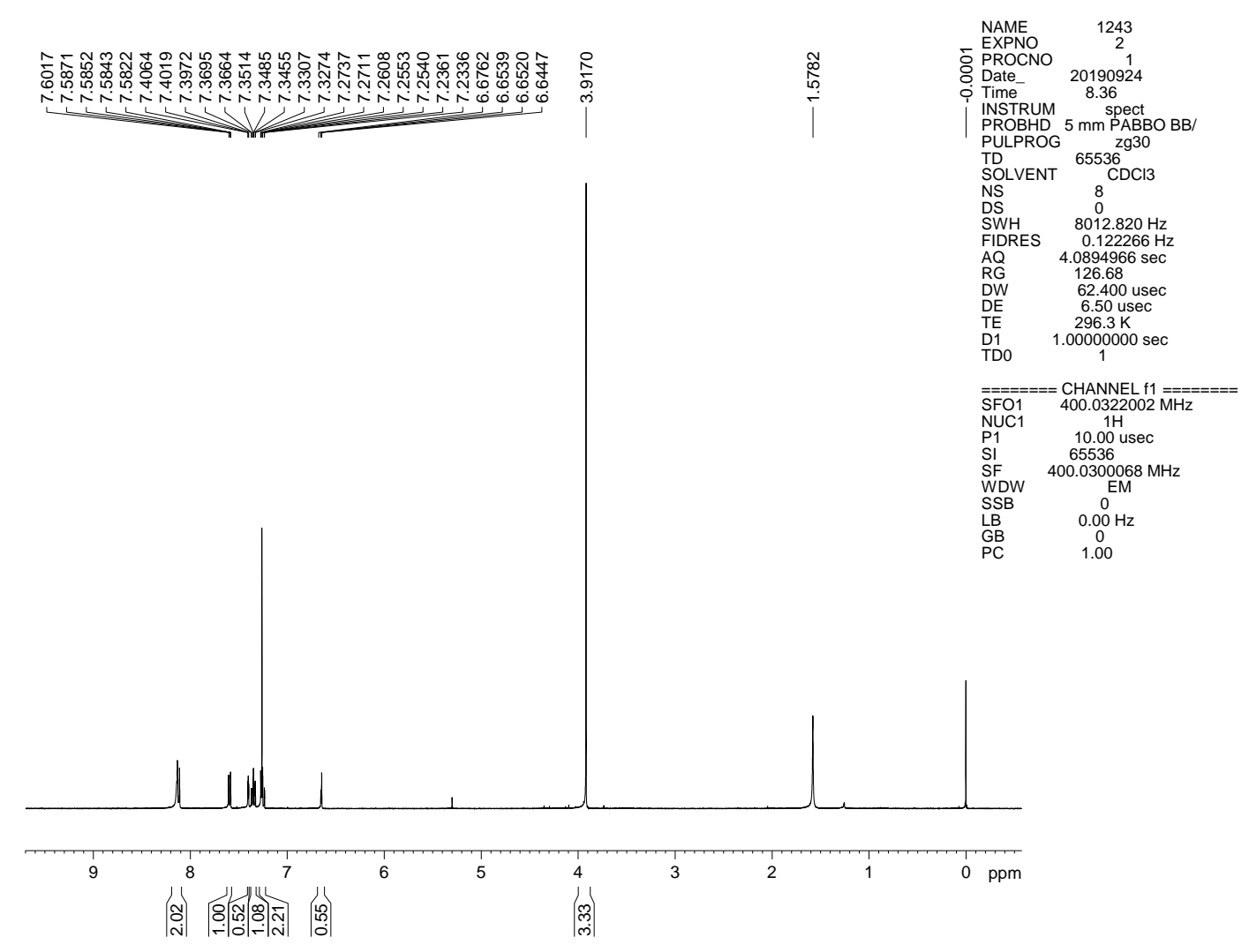

Figure S55 ${ }^{1} \mathrm{H}$ NMR (400 MHz, $\left.\mathrm{CDCl}_{3}\right)$ of Compound [D]-1a with $48 \%$ D Incorporation

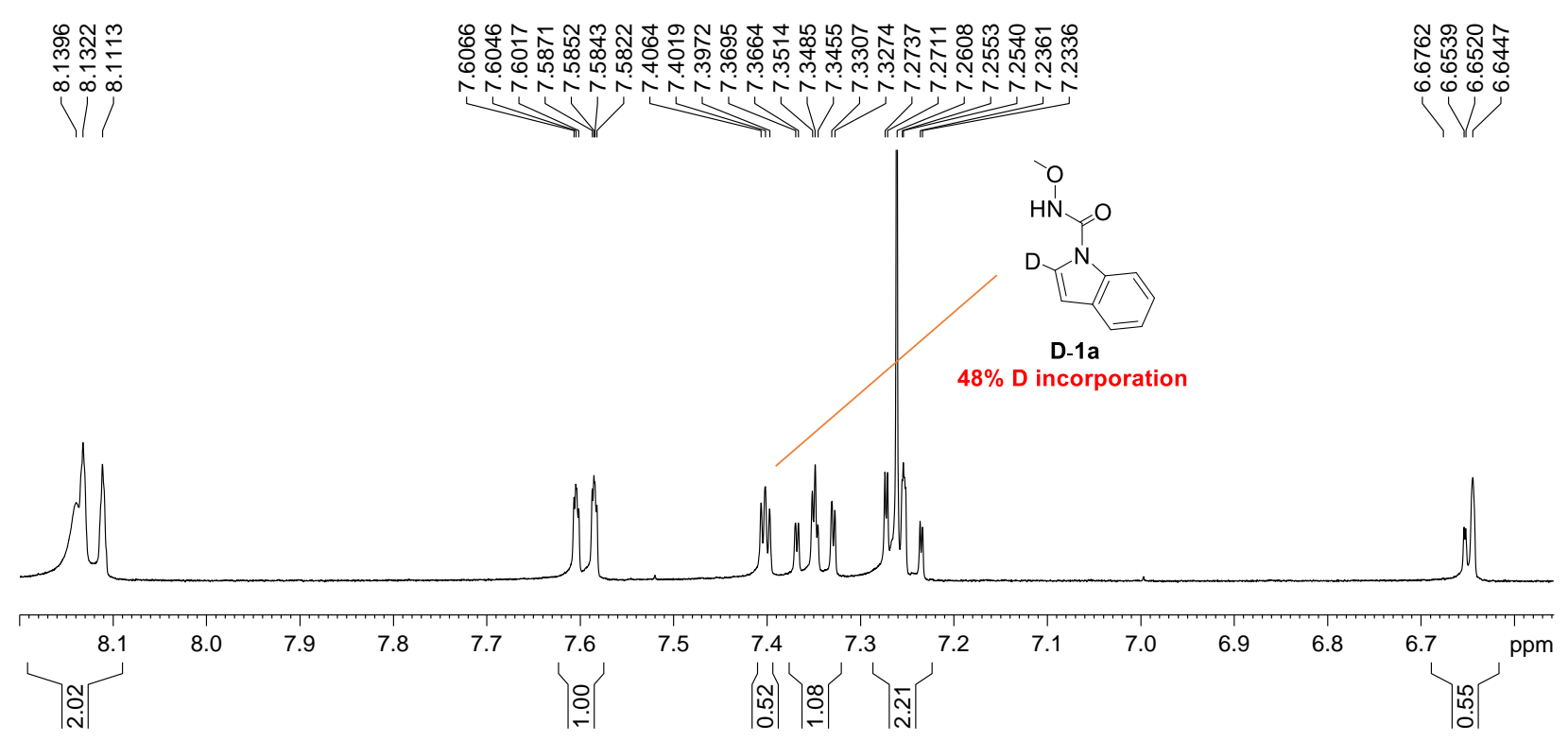

Figure S56 Expanded ${ }^{1} \mathrm{H}$ NMR (400 MHz, $\mathrm{CDCl}_{3}$ ) of Compound [D]-1a with 48\% D Incorporation 
8. UV-vis spectra of compounds $2 a-n$ and $3 a-d$
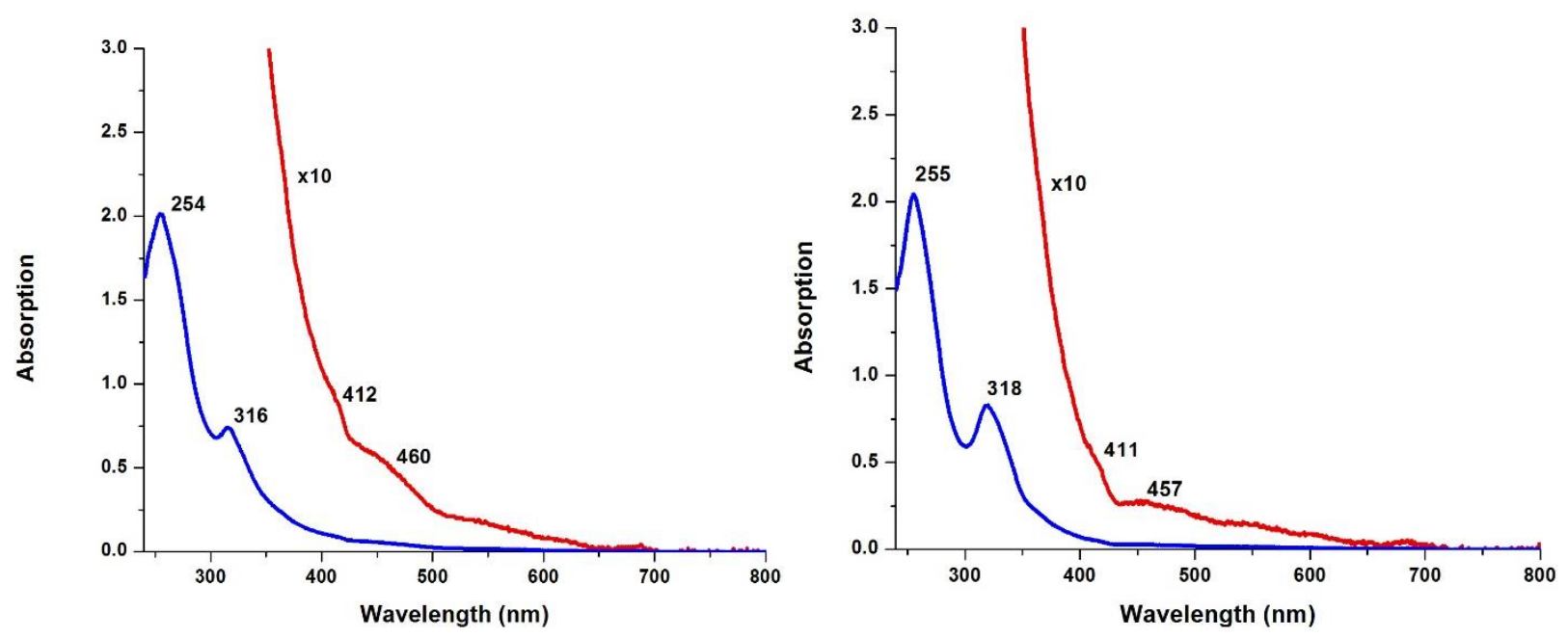

Figure S57 UV-vis absorption of compound 2a

Figure S58 UV-vis absorption of compound $2 b$
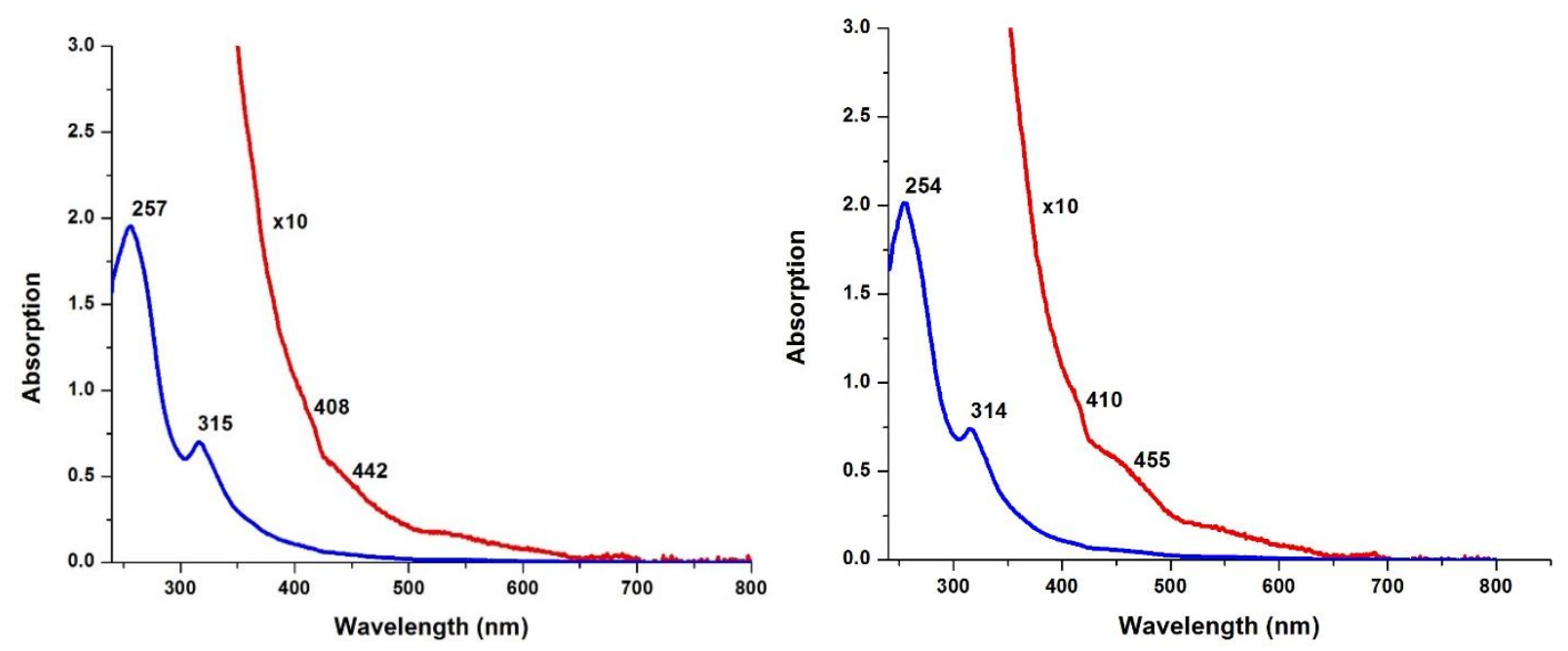

Figure S59 UV-vis absorption of compound 2c

Figure S60 UV-vis absorption of compound 2d 


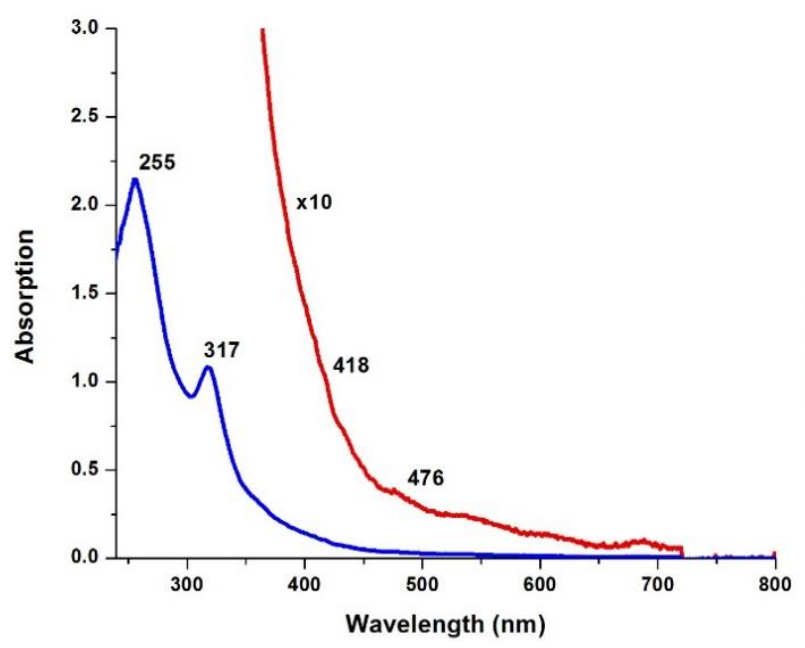

Figure S61 UV-vis absorption of compound 2e

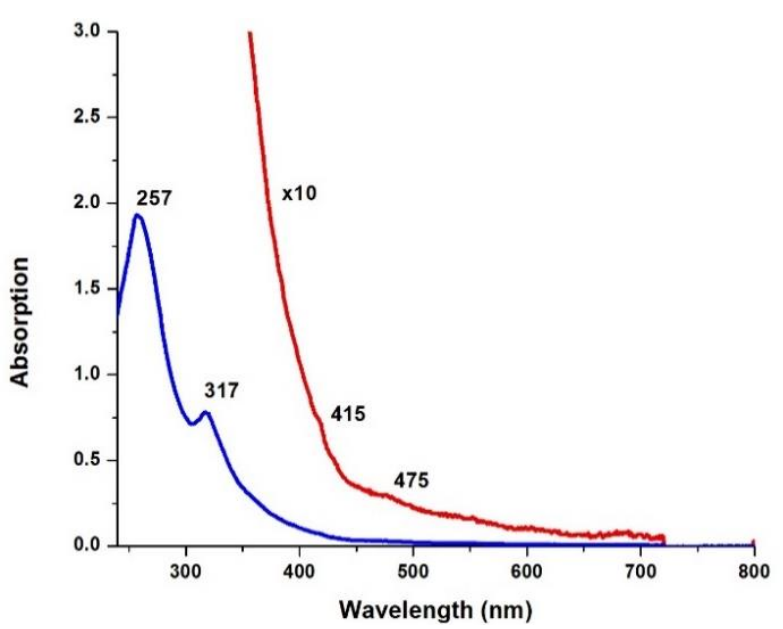

Figure S62 UV-vis absorption of compound $2 f$

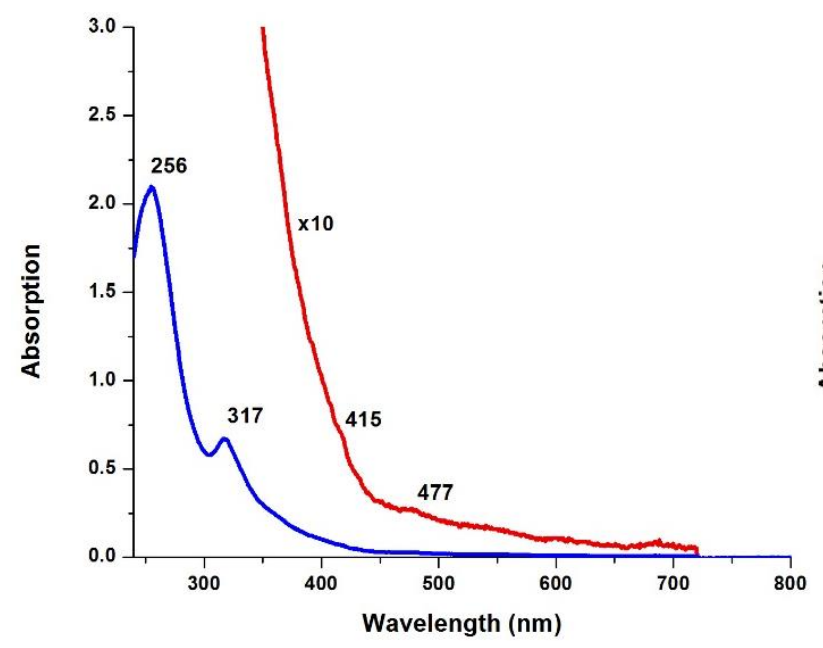

Figure S63 UV-vis absorption of compound $2 \mathrm{~g}$

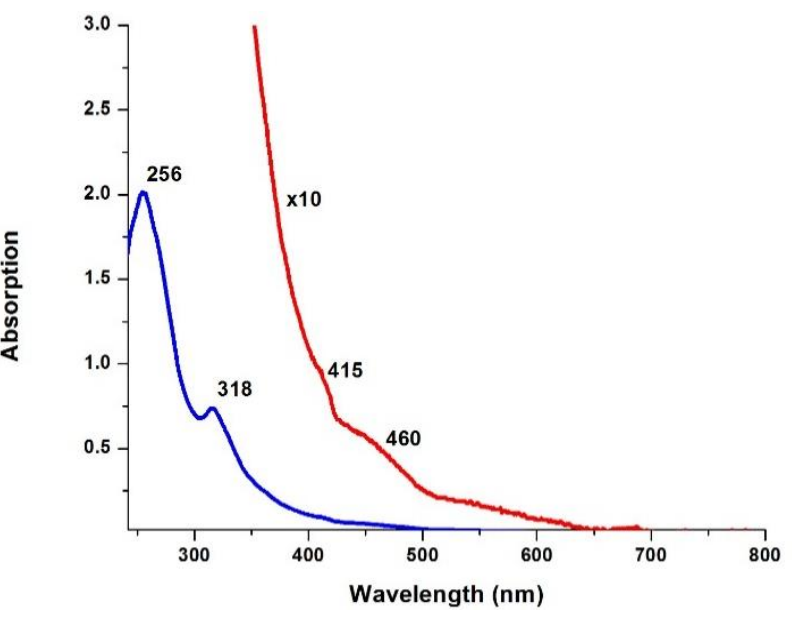

Figure S64 UV-vis absorption of compound $2 \mathrm{~h}$ 

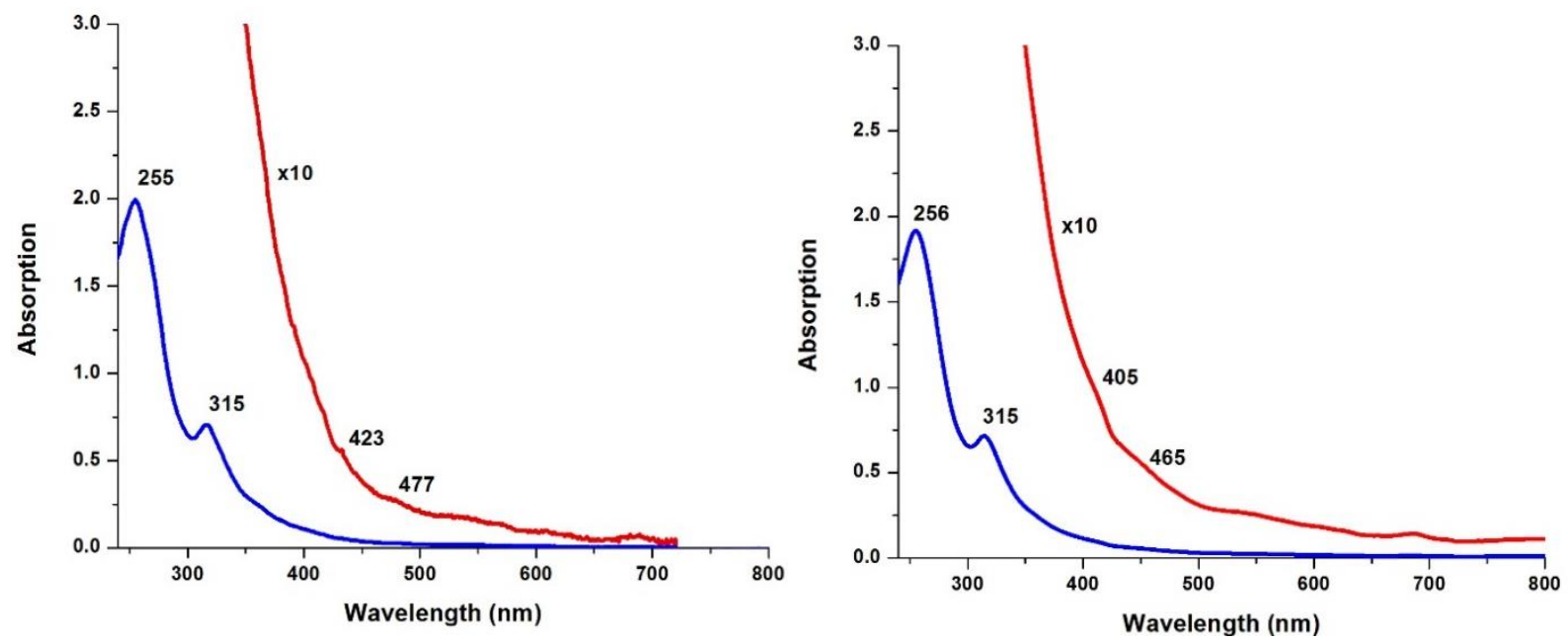

Figure S65 UV-vis absorption of compound $2 i$

Figure S66 UV-vis absorption of compound 2j
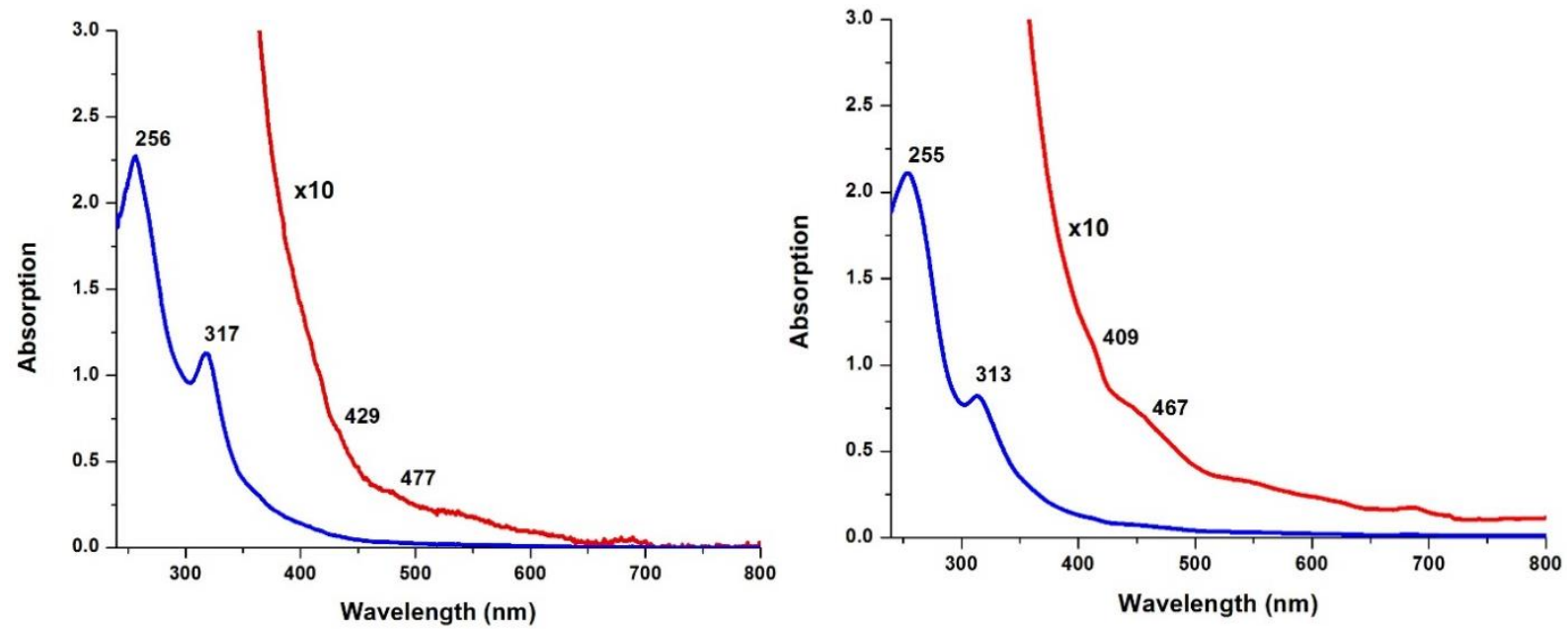

Figure S67 UV-vis absorption of compound $2 \mathrm{k}$

Figure S68 UV-vis absorption of compound 2I 


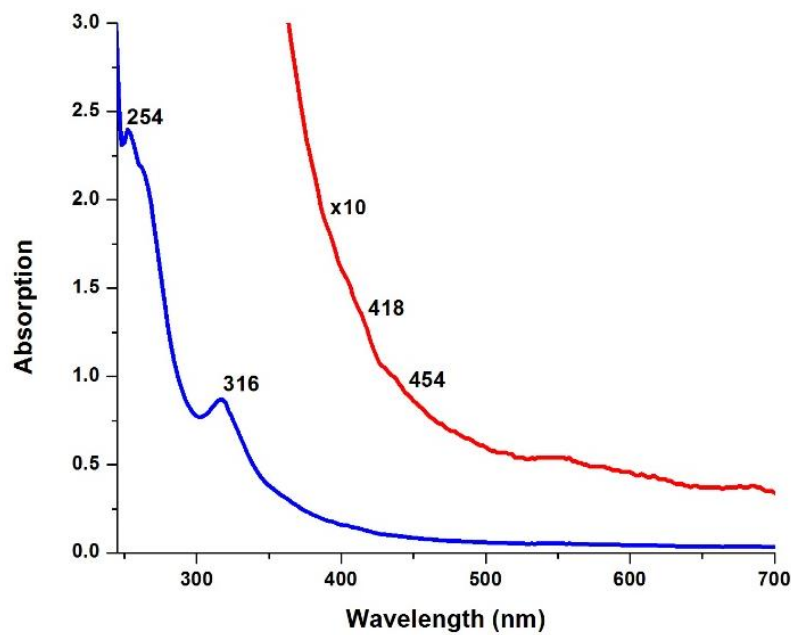

Figure S69 UV-vis absorption of compound $2 \mathrm{~m}$

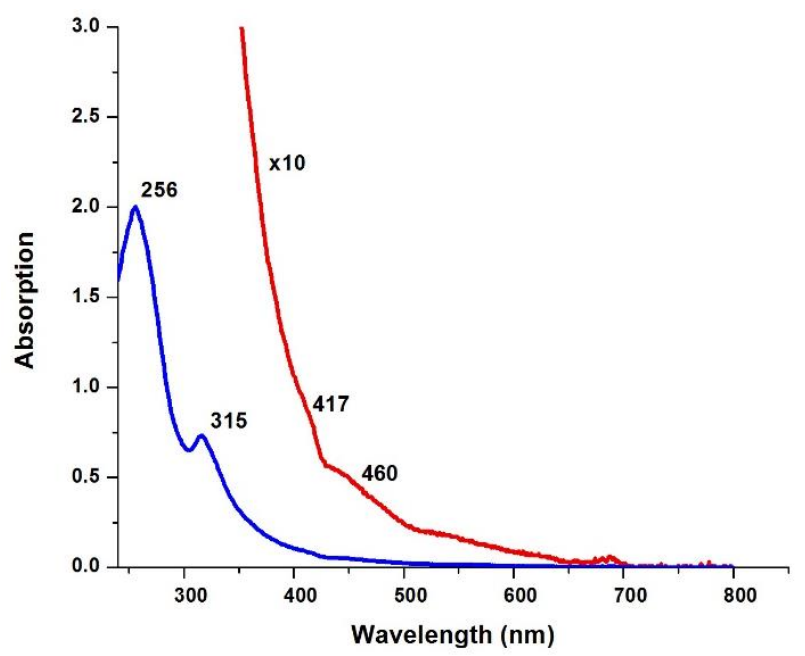

Figure S70 UV-vis absorption of compound 2n

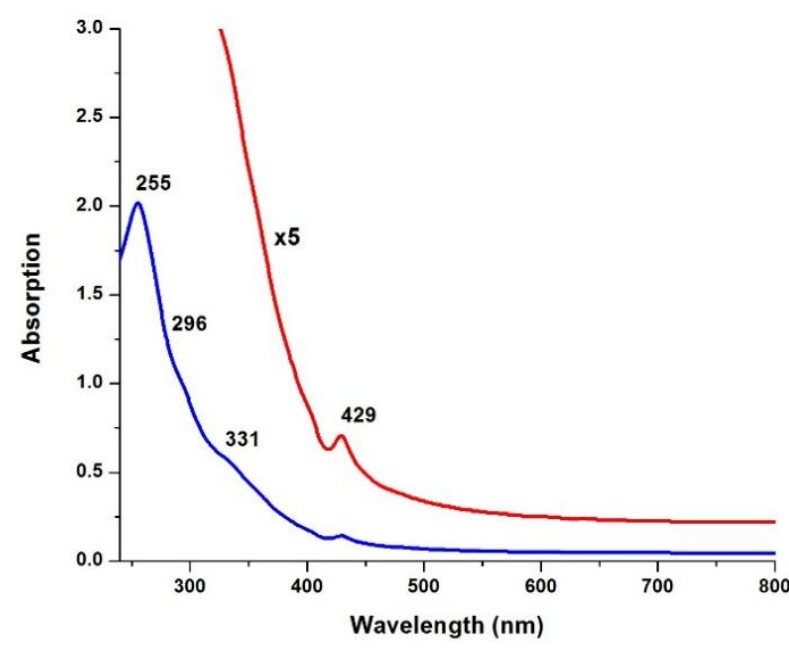

Figure S71 UV-vis absorption of compound 3a

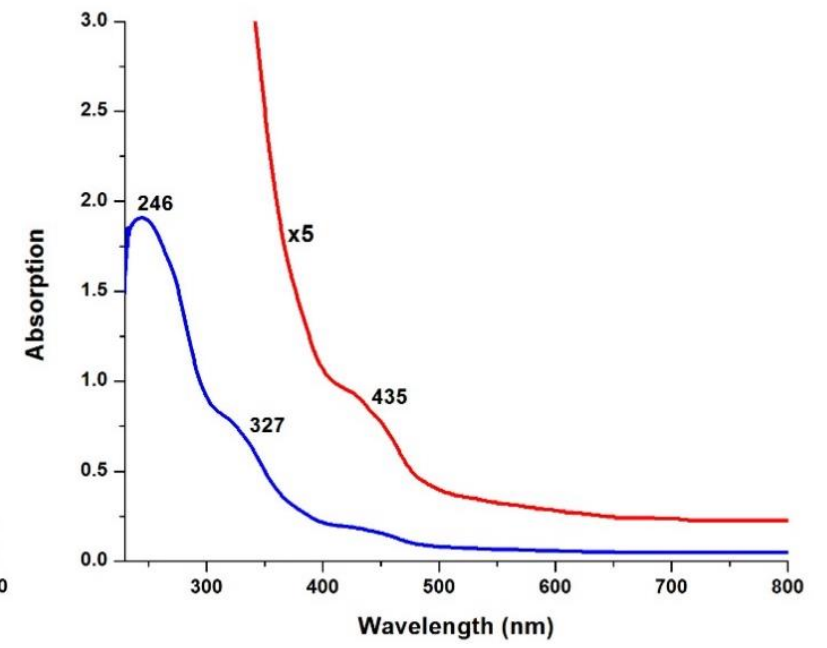

Figure S72 UV-vis absorption of compound 3b 


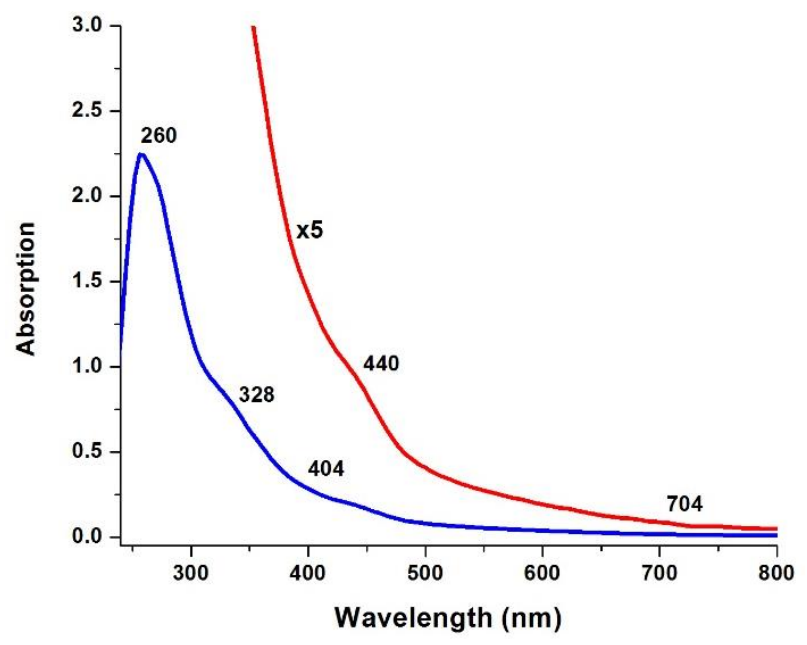

Figure S73 UV-vis absorption of compound 3c

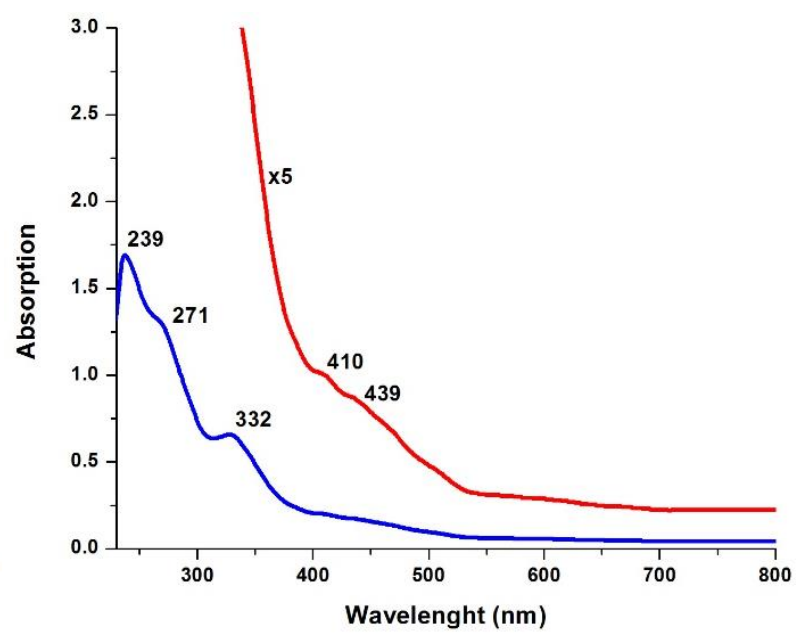

Figure S74 UV-vis absorption of compound 3d 
9. CV of compounds $2 a-n$ and $3 a-d$

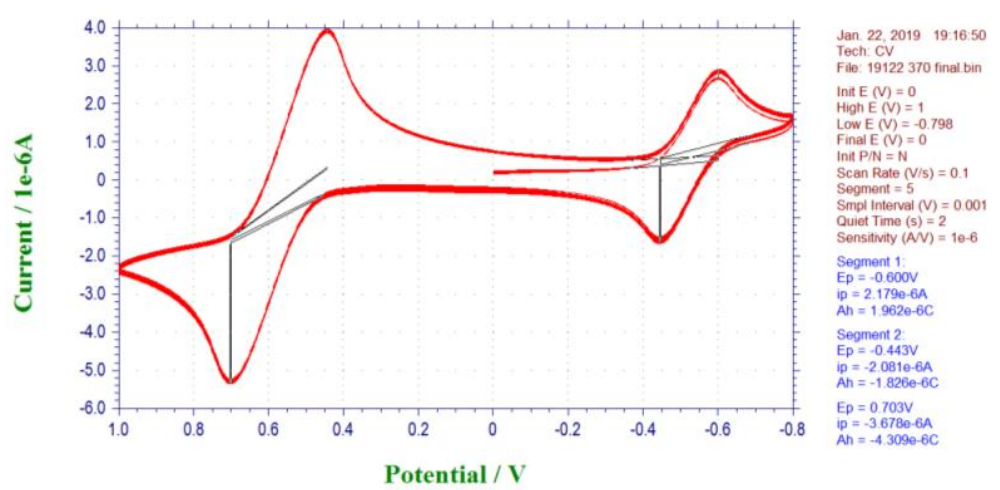

Figure S75. Cyclic voltammogram of compound 2a (scanning rate: $100 \mathrm{mV} \mathrm{s}^{-1}$ )

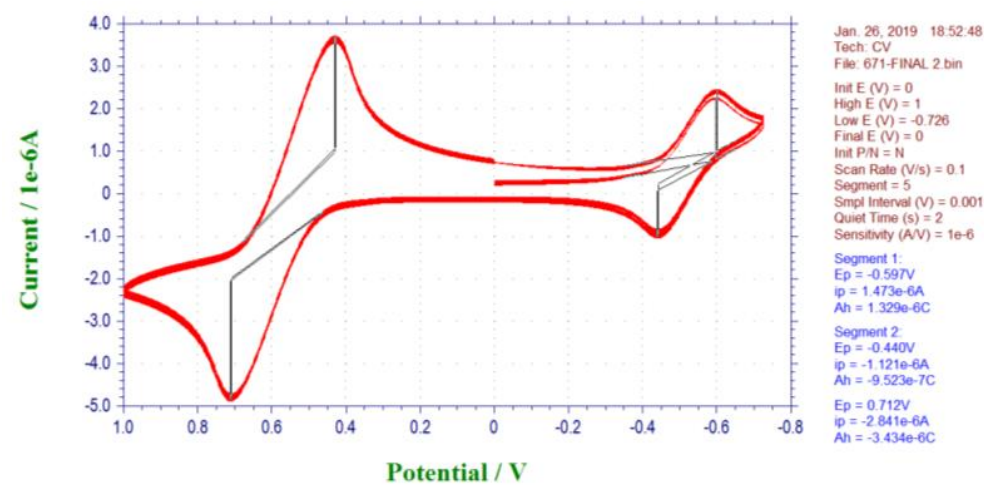

Figure S76. Cyclic voltammogram of compound $2 \mathrm{~b}$ (scanning rate: $100 \mathrm{mV} \mathrm{s}^{-1}$ )

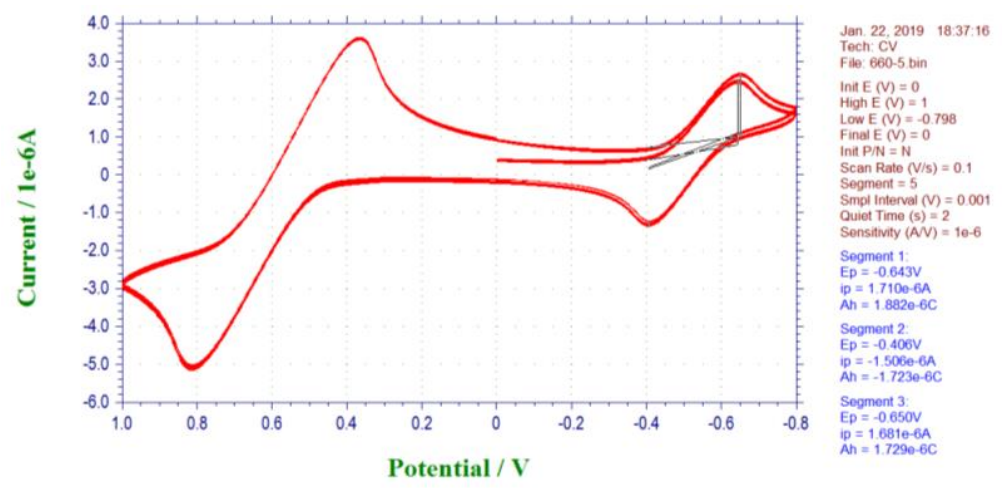

Figure S77. Cyclic voltammogram of compound 2c (scanning rate: $100 \mathrm{mV} \mathrm{s}^{-1}$ ) 


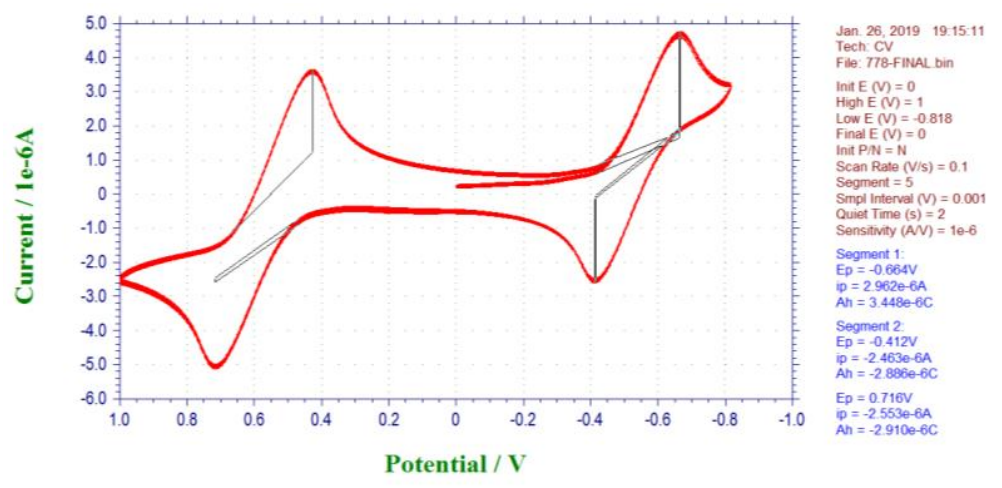

Figure S78. Cyclic voltammogram of compound $2 \mathrm{~d}$ (scanning rate: $100 \mathrm{mV} \mathrm{s}^{-1}$ )

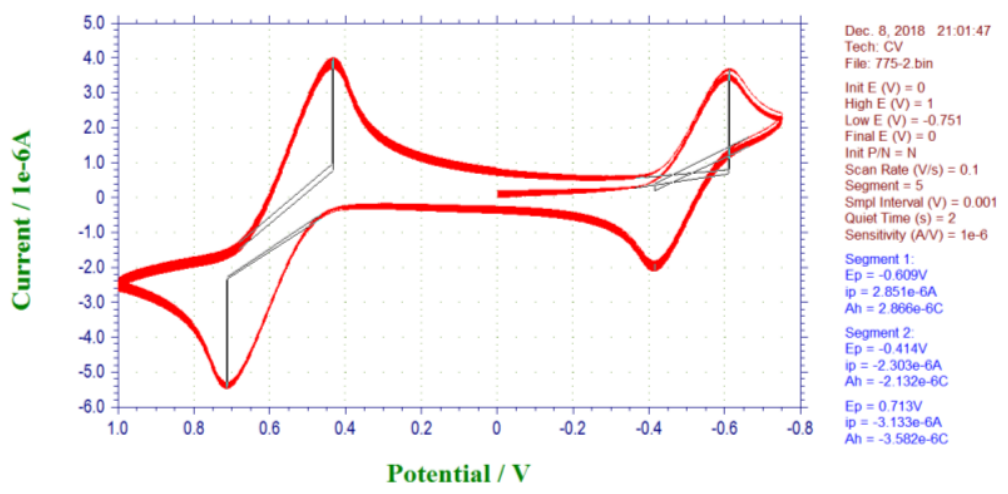

Figure S79. Cyclic voltammogram of compound 2e (scanning rate: $\mathrm{mV} \mathrm{s}^{-1}$ )

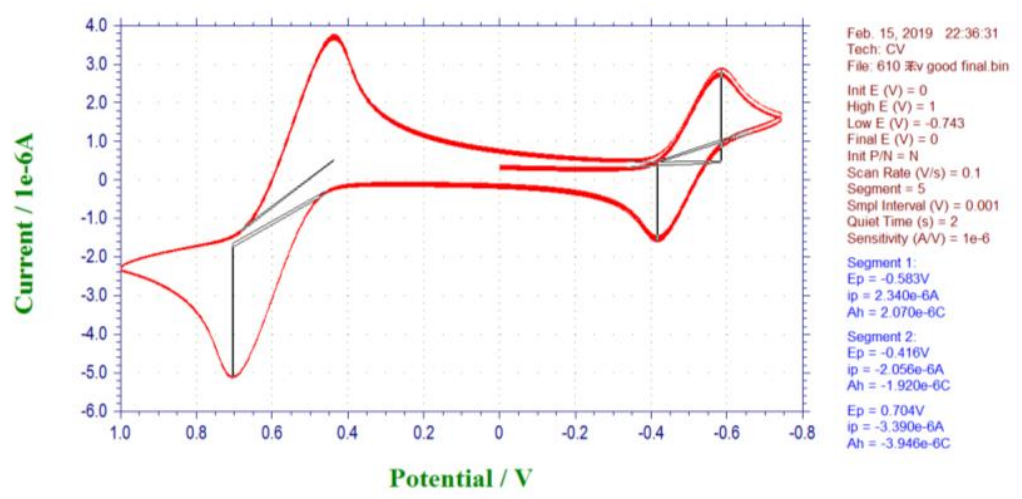

Figure S80. Cyclic voltammogram of compound $2 \mathrm{f}$ (scanning rate: $100 \mathrm{mV} \mathrm{s}^{-1}$ ) 


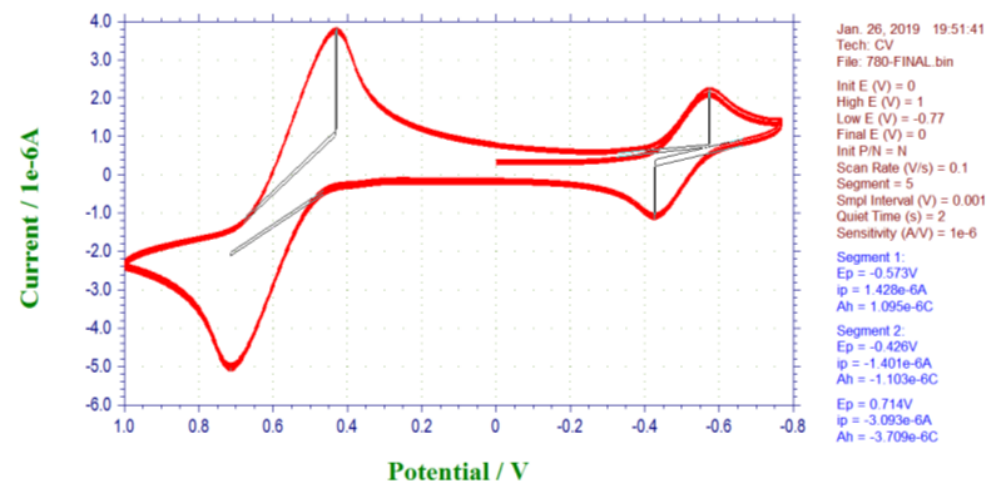

Figure S81. Cyclic voltammogram of compound $2 \mathrm{~g}$ (scanning rate: $100 \mathrm{mV} \mathrm{s}^{-1}$ )

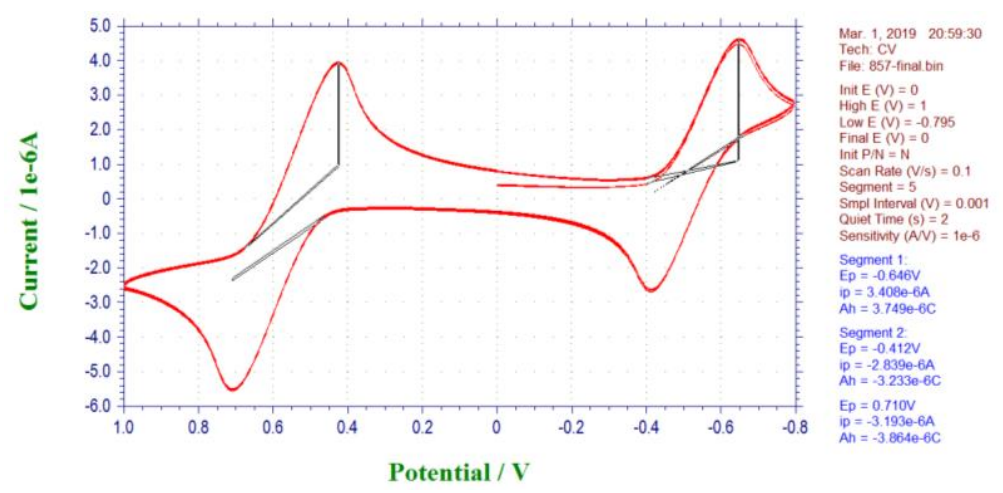

Figure S82. Cyclic voltammogram of compound $2 \mathrm{~h}$ (scanning rate: $100 \mathrm{mV} \mathrm{s}^{-1}$ )

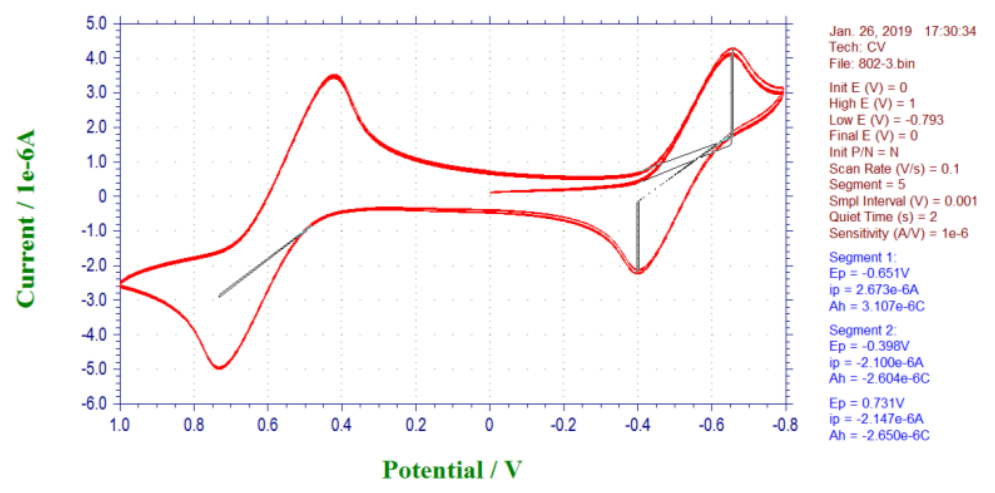

Figure S83. Cyclic voltammogram of compound $2 \mathrm{i}$ (scanning rate: $100 \mathrm{mV} \mathrm{s}^{-1}$ ) 


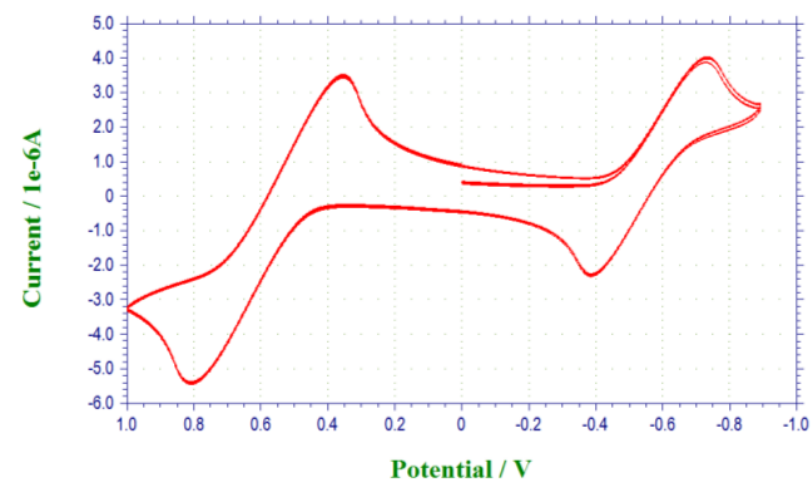

June 10, $2019 \quad 19.23 .43$

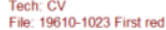
$\operatorname{Inn} E(N)=0$
High $E M()=1$ Low $E M=-0893$ Final $E(M)=0$ Scan Rate $(\mathrm{N} / \mathrm{s})$ Segment $=5$
mpli Interval $(V)=0.001$ Seniet Time (s) = 2 Segment 1: Segment 3 . Segment 5

Figure S84. Cyclic voltammogram of compound $2 \mathrm{j}$ (scanning rate: $100 \mathrm{mV} \mathrm{s}^{-1}$ )

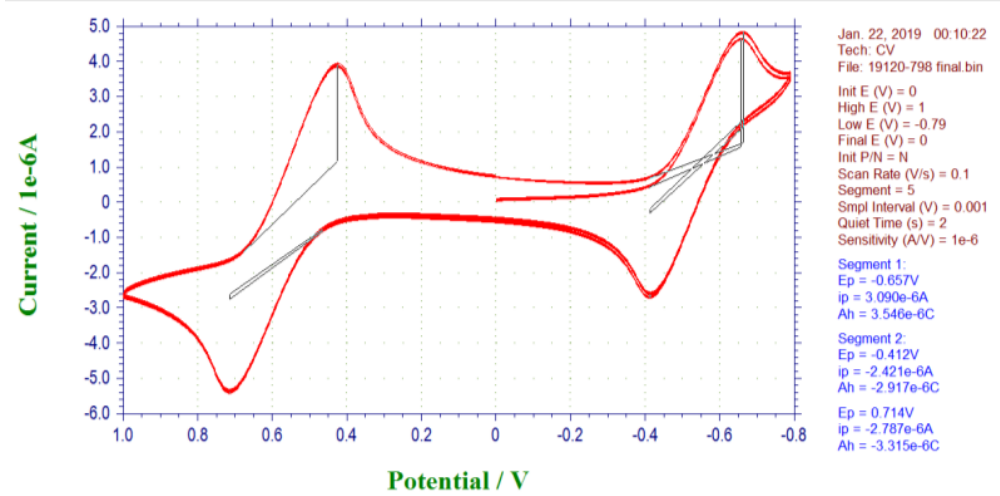

Figure S85. Cyclic voltammogram of compound $2 \mathrm{k}$ (scanning rate: $100 \mathrm{mV} \mathrm{s}^{-1}$ )

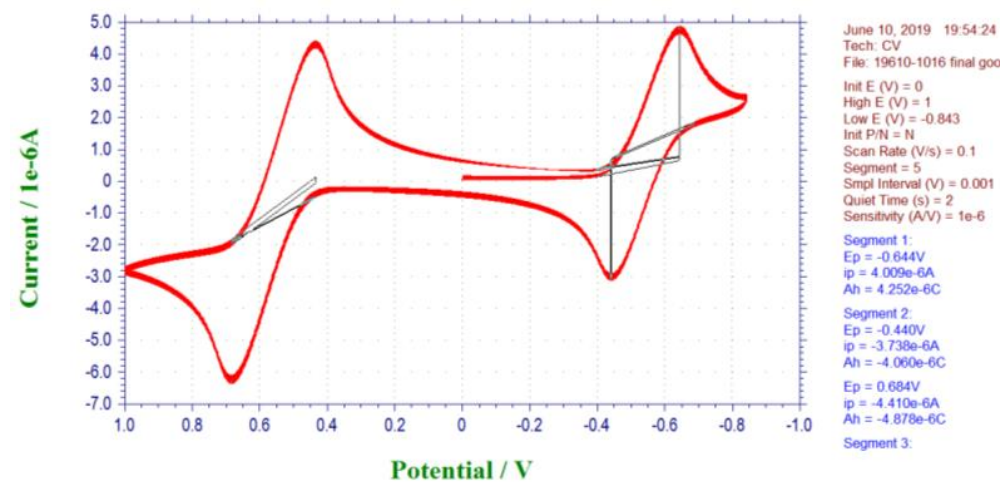

Figure S86. Cyclic voltammogram of compound 21 (scanning rate: $100 \mathrm{mV} \mathrm{s}^{-1}$ ) 


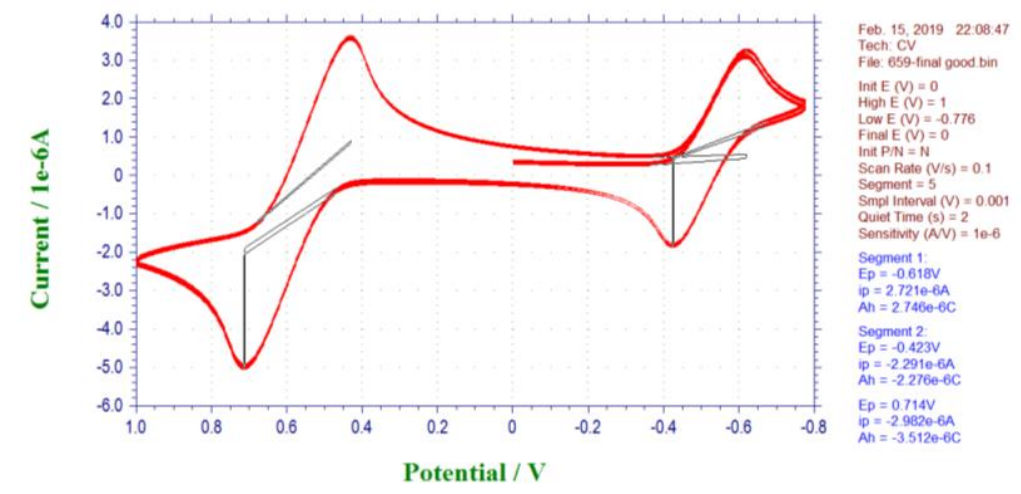

Figure S87. Cyclic voltammogram of compound $2 \mathrm{~m}$ (scanning rate: $\mathrm{mV} \mathrm{s}^{-1}$ )

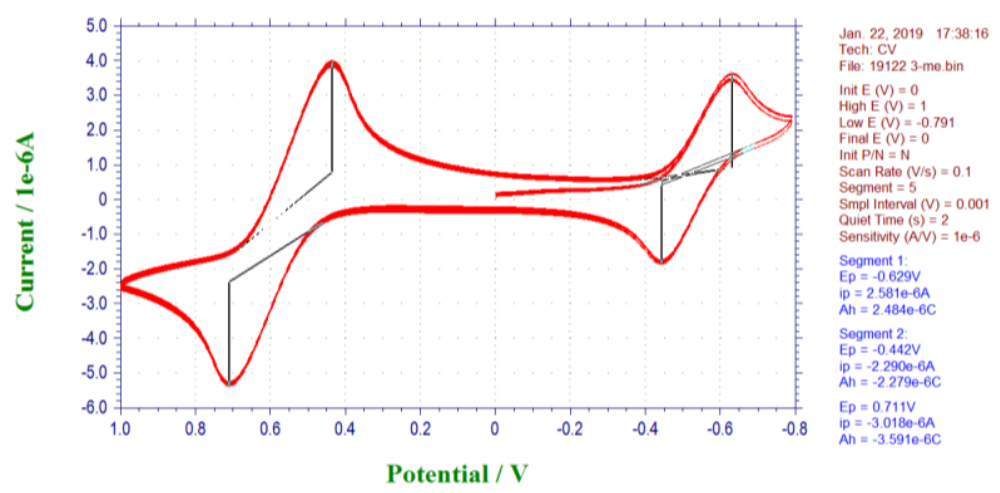

Figure S88. Cyclic voltammogram of compound $2 \mathrm{n}$ (scanning rate: $100 \mathrm{mV} \mathrm{s}^{-1}$ )

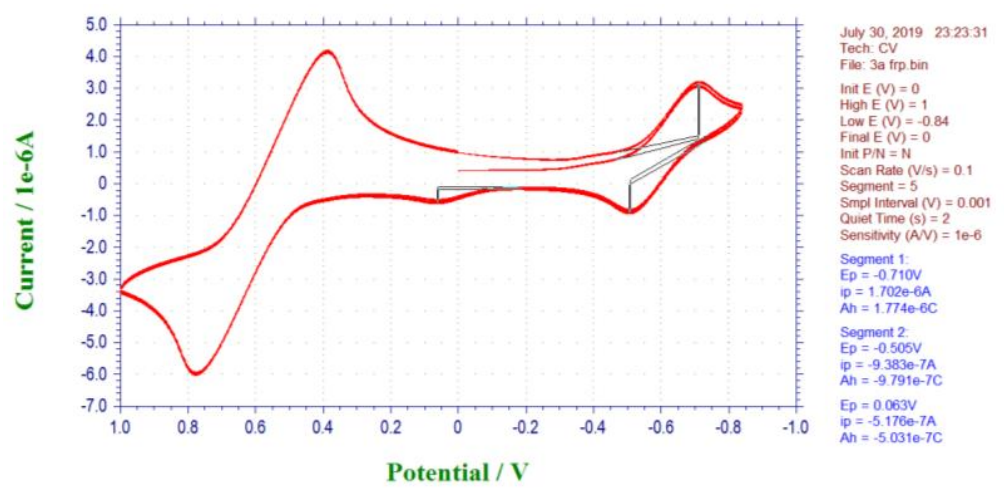

Figure S89. Cyclic voltammogram of compound 3a (scanning rate: $100 \mathrm{mV} \mathrm{s}^{-1}$ ) 

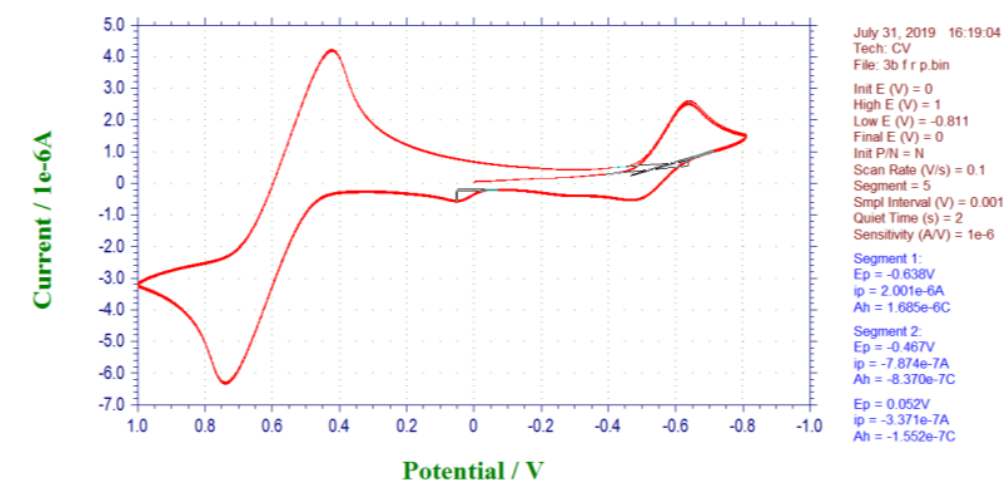

Figure S90. Cyclic voltammogram of compound $3 \mathrm{~b}$ (scanning rate: $100 \mathrm{mV} \mathrm{s}^{-1}$ )

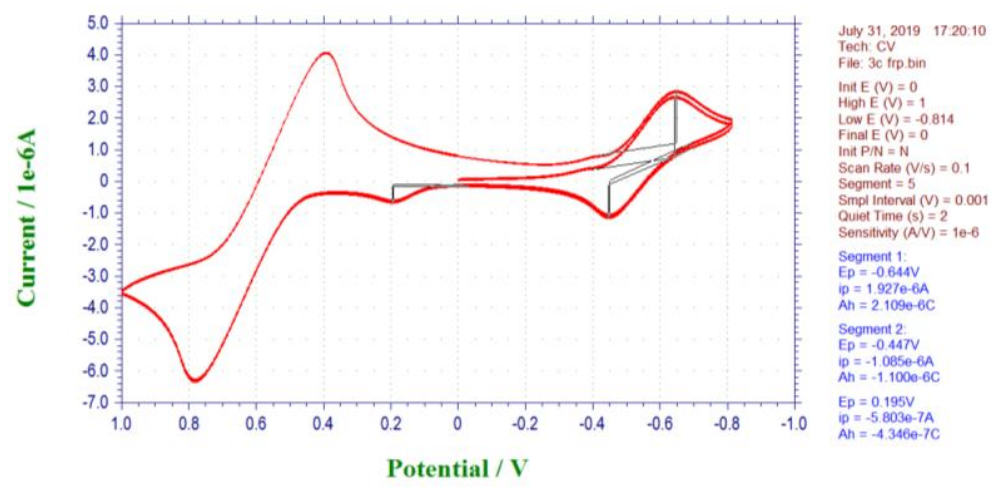

Figure S91. Cyclic voltammogram of compound 3c (scanning rate: $100 \mathrm{mV} \mathrm{s}^{-1}$ )

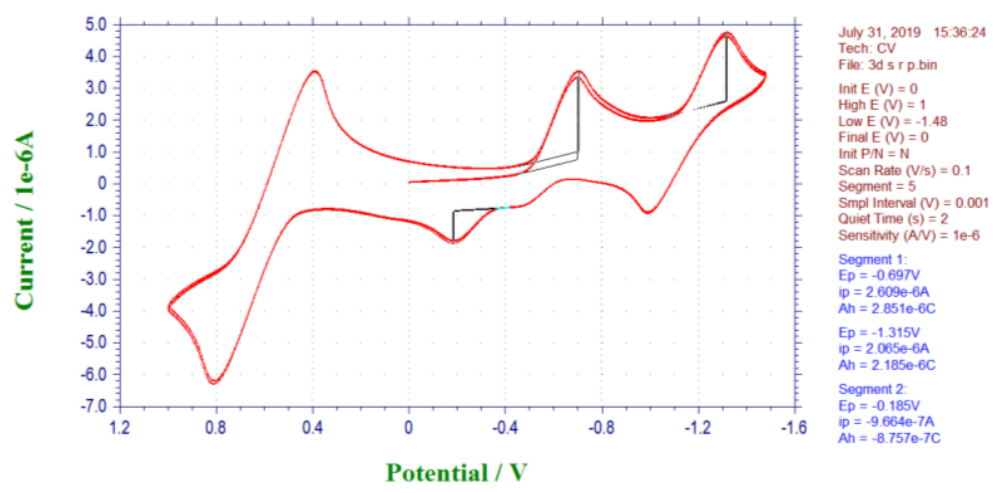

Figure S92. Cyclic voltammogram of compound 3d (scanning rate: $100 \mathrm{mV} \mathrm{s}^{-1}$ ) 


\section{X-Ray data of compound 2e}

Black block crystals of $2 \mathrm{e}$ were obtained by slow evaporation of a saturated solution in chloroform at room temperature. Single-crystal X-ray diffraction data were collected on a diffractometer (Gemini S Ultra, Agilent Technologies) equipped with a CCD area detector using graphite-monochromated $\mathrm{Cu} \mathrm{K \alpha}$ radiation $(\lambda=1.54184 \AA)$ in the scan range $8.18^{\circ}<2 \theta<147.91^{\circ}$. The structure was solved with direct methods using SHELXS-97 and refined with full-matrix leastsquares refinement using the SHELXL-97 program within OLEX2.

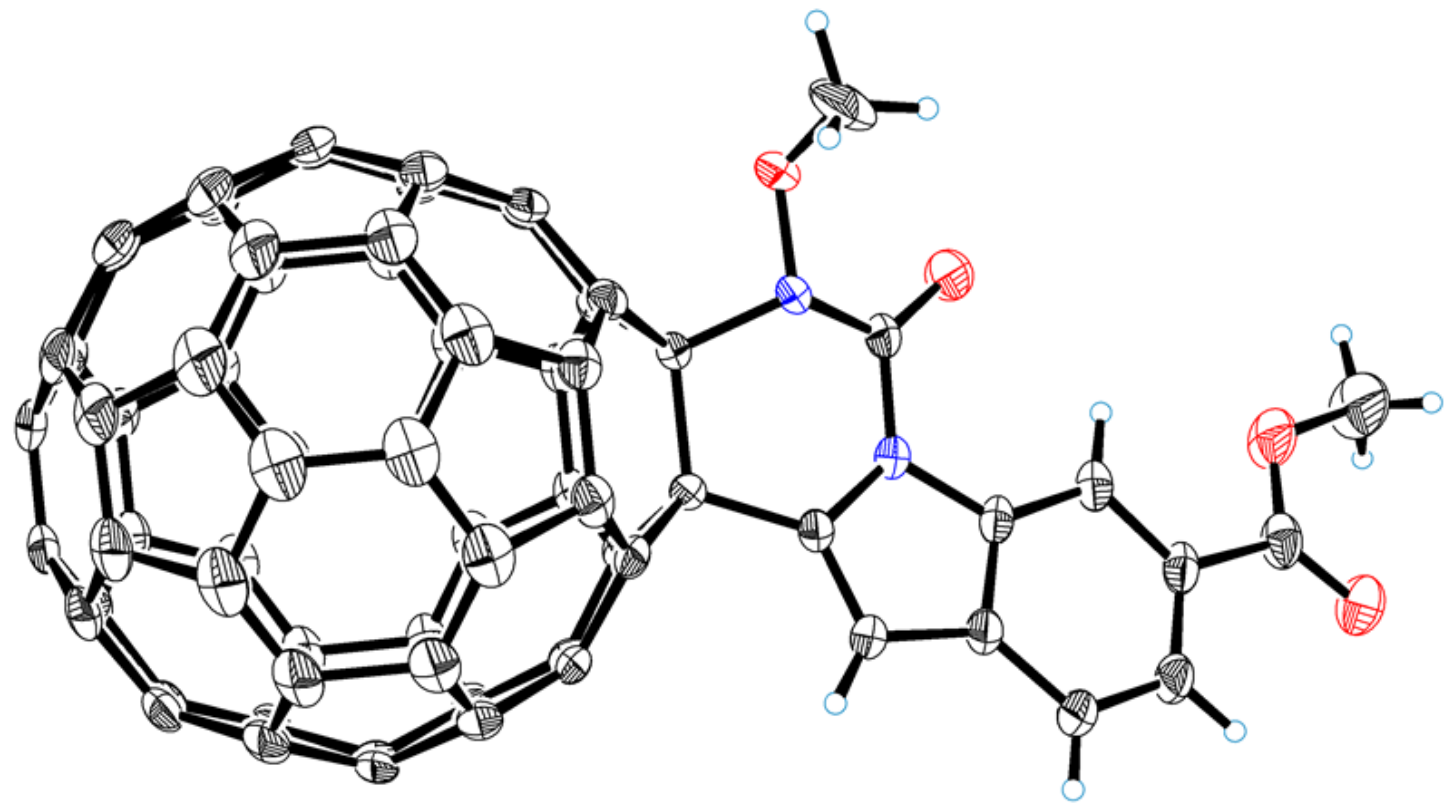

Figure S93 ORTEP diagrams of $2 \mathrm{e}$ with $30 \%$ thermal ellipsoids. The chloroform molecule is omitted for clarity. 


\begin{tabular}{|c|c|}
\hline \multicolumn{2}{|c|}{ Table 1 Crystal data and structure refinement for $2 \mathrm{e}}$. \\
\hline Identification code & $2 e$ \\
\hline Empirical formula & $\mathrm{C}_{74} \mathrm{H}_{12} \mathrm{Cl}_{6} \mathrm{~N}_{2} \mathrm{O}_{4}$ \\
\hline Formula weight & 1201.89281 \\
\hline Temperature/K & $291(2)$ \\
\hline Crystal system & monoclinic \\
\hline Space group & $\mathrm{P} 2_{1} / \mathrm{n}$ \\
\hline $\mathrm{a} / \AA ̊ \AA$ & $10.02120(10)$ \\
\hline $\mathrm{b} / \AA ̊ \AA$ & $30.3580(5)$ \\
\hline$c / \AA ̊$ & $15.4145(2)$ \\
\hline$\alpha /^{\circ}$ & 90 \\
\hline$\beta /^{\circ}$ & $90.7190(10)$ \\
\hline$\gamma /{ }^{\circ}$ & 90 \\
\hline Volume $/ \AA^{3}$ & $4689.08(11)$ \\
\hline$Z$ & 4 \\
\hline$\rho_{\text {calc }} \mathrm{g} / \mathrm{cm}^{3}$ & 1.708 \\
\hline$\mu / \mathrm{mm}^{-1}$ & 3.895 \\
\hline$F(000)$ & 2416.0 \\
\hline Crystal size $/ \mathrm{mm}^{3}$ & $0.260 \times 0.220 \times 0.210$ \\
\hline Radiation & $\operatorname{CuK} \alpha(\lambda=1.54184)$ \\
\hline $2 \Theta$ range for data collection $/^{\circ}$ & 8.176 to 147.908 \\
\hline Index ranges & $\begin{array}{l}-12 \leqslant h \leqslant 7,-36 \leqslant k \leqslant 37,-18 \leqslant 1 \leqslant \\
18\end{array}$ \\
\hline Reflections collected & 18893 \\
\hline Independent reflections & $9242\left[R_{\text {int }}=0.0495, R_{\text {sigma }}=0.0561\right]$ \\
\hline Data/restraints/parameters & $9242 / 0 / 777$ \\
\hline Goodness-of-fit on $F^{2}$ & 1.079 \\
\hline Final $R$ indexes $[I>=2 \sigma(I)]$ & $R_{1}=0.0986, w R_{2}=0.2322$ \\
\hline Final $\mathrm{R}$ in dexes [all data] & $R_{1}=0.1207, w R_{2}=0.2497$ \\
\hline Largest diff. peak/hole / e $\AA^{-3}$ & $0.95 /-0.95$ \\
\hline
\end{tabular}




\section{X-Ray data of compound 3d}

Black block crystals of 3d were obtained by slow evaporation of a saturated solution in carbon disulfide and methanol at $4{ }^{\circ} \mathrm{C}$. The $\mathrm{X}$-ray diffraction of $\mathbf{3 d}$ was performed at $100 \mathrm{~K}$ using the wavelength of $0.71073 \AA$ with a CCD detector at the beamline BL17B of the Shanghai Synchrotron Radiation Facility (SSRF). The multiscan method was used for absorption corrections. The structure was solved by direct method and refined with full-matrix least-squares refinement using the SHELXL-2016/6 within OLEX2.

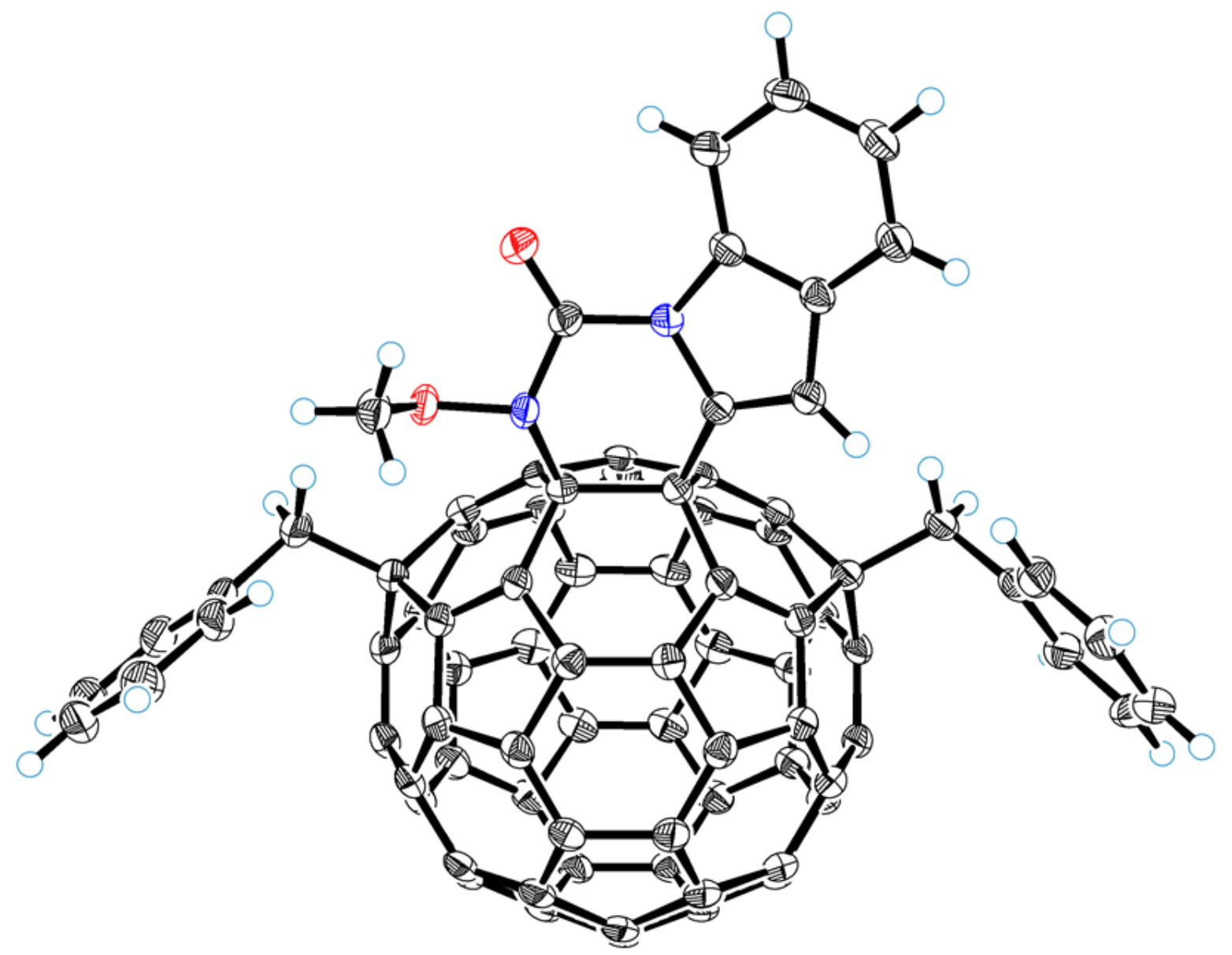

Figure S94 ORTEP diagrams of $3 d$ with $60 \%$ thermal ellipsoids. The carbon disulfide molecule is omitted for clarity. 


\begin{tabular}{|c|c|}
\hline \multicolumn{2}{|c|}{ Table 2 Crystal data and structure refinement for $3 \mathrm{~d}$. } \\
\hline Identification code & $3 d$ \\
\hline Empirical formula & $\mathrm{C}_{85} \mathrm{H}_{22} \mathrm{~N}_{2} \mathrm{O}_{2} \mathrm{~S}_{2}$ \\
\hline Formula weight & 1167.17 \\
\hline Temperature/K & $100(2)$ \\
\hline Crystal system & triclinic \\
\hline Space group & P-1 \\
\hline $\mathrm{a} / \AA ̊ \AA$ & 10.933 \\
\hline $\mathrm{b} / \AA ̊ \AA$ & 13.657 \\
\hline$c / A ̊$ & 17.997 \\
\hline$\alpha /^{\circ}$ & 69.90 \\
\hline$\beta /{ }^{\circ}$ & 73.57 \\
\hline$y /{ }^{\circ}$ & 88.77 \\
\hline Volume/ $/ \AA^{3}$ & 2412.0 \\
\hline $\mathrm{Z}$ & 2 \\
\hline$\rho_{\text {calc }} \mathrm{g} / \mathrm{cm}^{3}$ & 1.607 \\
\hline$\mu / \mathrm{mm}^{-1}$ & 0.179 \\
\hline $\mathrm{F}(000)$ & 1188.0 \\
\hline Crystal size $/ \mathrm{mm}^{3}$ & $0.02 \times 0.01 \times 0.01$ \\
\hline Radiation & synchrotron \\
\hline $2 \Theta$ range for data collection $/^{\circ}$ & 5.434 to 54.218 \\
\hline Reflections collected & 9612 \\
\hline Independent reflections & $9612\left[R_{\text {int }}=0.0390, R_{\text {sigma }}=0.0320\right]$ \\
\hline Data/restraints/parameters & $9612 / 0 / 821$ \\
\hline Goodness-of-fit on $F^{2}$ & 1.057 \\
\hline Final $R$ indexes $[I>=2 \sigma(I)]$ & $\mathrm{R}_{1}=0.0468, \mathrm{wR}_{2}=0.1277$ \\
\hline Final $R$ indexes [all data] & $R_{1}=0.0487, w R_{2}=0.1297$ \\
\hline Largest diff. peak/hole / e $\AA^{-3}$ & $0.60 /-0.52$ \\
\hline
\end{tabular}

